\title{
TAU FUNCTIONS FOR THE DIRAC OPERATOR IN THE EUCLIDEAN PLANE
}

\author{
JOHN PALMER
}

\begin{abstract}
In this paper, the $\tau$-functions introduced by M. Sato, M. Miwa, and $T$. Jimbo in their study of monodromy preserving deformations of the Dirac equation are rigorously identified as determinants of singular Dirac operators. The singular Dirac operators have branched functions in their domains that reflect the monodromy in the deformation theory. The principal result is a new formula for the $\tau$-function, obtained by trivializing a suitable determinant bundle, that can be simply related to the deformation theory and which may also be computed in the transfer matrix formalism. These two different ways of understanding the $\tau$-function provide the link between the deformation theory and the quantum field theory significance of $\tau$-function as a correlation function. This connection is the central result of the Sato-Miwa-Jimbo theory of Holonomic Fields.
\end{abstract}

Introduction. In this paper we develop a new version of the SatoMiwa-Jimbo theory of $\tau$-functions for the Euclidean Dirac operator acting in the plane $\mathbf{R}^{2}$. Before attempting to explain the features of this analysis which are new it will be useful to recall the original setting and results in [14]. In $1973 \mathrm{Wu}, \mathrm{McCoy}$, Tracy, and Barouch announced results in Physical Review Letters [1] for the scaling limit of the two point correlation function of the two dimensional Ising model. In [19] and [20] they published a full account of the remarkable result that this scaled correlation could be expressed in terms of a Painlevé function of the third kind. In a series of five long papers titled "Holonomic Quantum Fields I-V" published in the years 1978-1980, the mathematicians M. Sato, T. Miwa, and M. Jimbo (SMJ henceforth) revealed that the WMTB result was a specal case of a more general phenomena. There is a class of two dimensional quantum field theories whose correlation functions (i.e., Schwinger functions) could be expressed in terms of the solutions to nonlinear equations associated with monodromy preserving deformations of linear differential equations. SMJ named these quantum fields "Holonomic Quantum Fields" in reference to the intimate connection they have with holonomic systems of linear differential equations. A holonomic system of linear differential equations is one that is "maximally overdetermined" in a 
technical sense and one of the tools introduced by SMJ in the study of monodromy preserving deformation theory is the introduction of an appropriate associated holonomic system. For example, they considered the Dirac equation in $\mathbf{R}^{2}$ (with a mass term)

$$
D(m) \psi=0,
$$

and studied solutions to this equation which have isolated singularities at points $a_{j}$ for $j=1, \ldots, n$, where they are "branched" with monodromy $e^{-2 \pi i l_{j}}$. Of course, the solutions $\psi$ to such a problem ought to be considered as functions on the simply connected covering space of $\mathbf{R}^{2} \backslash\left\{a_{1}, \ldots, a_{n}\right\}$. In [14-III] SMJ showed that if one confined oneself to the solutions of this problem which are in $L^{2}\left(\mathbf{R}^{2}\right)$ then the space of solutions is $n$-dimensional. They construct a canonical basis $\left\{w_{1}, \ldots, w_{n}\right\}$ for this space and they show that the vector $\left[w_{1}, \ldots, w_{n}\right]$ can be thought of as a flat section for a "Dirac compatible connection" on the maps from $\mathbf{R}^{2} \backslash\left\{a_{1}, \ldots, a_{n}\right\}$ into $\mathbf{C}^{2 n}$. The holonomy of this connection about the point $a_{j}$ is simply related to the monodromy $e^{-2 \pi i l_{j}}$. The elements of the canonical basis $w_{j}$ depend on the points $a_{1}, \ldots, a_{n}$, of course, and a basic technique in SMJ III is to extend the flat connection in the $\mathbf{R}^{2}$ variables to a connection in the $\left\{a_{1}, \ldots, a_{n}\right\}$ variables with respect to which the vector $\left[w_{1}, \ldots, w_{n}\right]$ remains a flat section. The zero curvature condition for this connection gives the deformation equations which SMJ associate to the monodromy preserving deformation of the Dirac equation.

What has this to do with quantum fields? In SMJ IV singular field operators $\phi_{l_{j}}\left(a_{j}\right)$ associated with the free Dirac field are introduced. SMJ refer to the vacuum expectations of such fields as $\tau$-functions defining $\tau(a)$ by:

$$
\tau(a)=\left\langle\phi_{l_{1}}\left(a_{1}\right) \cdots \phi_{l_{n}}\left(a_{n}\right)\right\rangle .
$$

By introducing Dirac fields in the expectation defining $\tau$, SMJ show that one obtains wave functions for the Dirac equation which can ultimately be related to the canonical wave functions introduced above. The systematic and clever introduction of local operator product expansions allows SMJ to recover the $\tau$-function from the local expansion coefficients of the wave functions. The field operators $\phi_{l}(a)$ introduced by SMJ are quite singular objects and a lot of effort has gone into trying to make rigorous mathematical sense of them. They turn out to be intimately related to the Federbush and massless Thirring fields and the field theories associated with these formal quantum fields 
have been rigorously constructed by S. Ruijsenaars [13] and by A. Carey, S. Ruijsenaars, and J. Wright [2]. These field theories have been constructed in the Minkowski regime-the $\tau$-functions (or more precisely, objects akin to the SMJ $\tau$-functions) should be the analytic continuations of the correlations to pure imaginary time but I do not believe that this connection has been established yet. In another direction lattice analogues for (Euclidean) holonomic quantum fields were introduced in [7] and the convergence to a continuum limit was proved (earlier, the SMJ analysis of the scaling limit for the Ising model was dealt with in [9]). More recently, R. Davey [3] established that the continuum limit of the lattice fields in [7] is associated with an SMJ style deformation theory. However, his results also show that the limiting $\tau$-function is not always the same as the $\tau$-function which SMJ associate with their $L^{2}$ deformation theory. The analysis in [3] is difficult and tedious. It involves constructing lattice precursors of the continuum objects (like wave functions and local expansions) that are part of the SMJ analysis and then showing these lattice objects scale to a continuum setting in which one can carry out the SMJ analysis. These results are instructive but one pays a high price in the computational complexity that attends working on the lattice.

In the following we will introduce a mathematically well-defined theory of $\tau$-functions which produces the $\tau$-functions to which the lattice $\tau$-functions scale. The approach we adopt by-passes the construction of the field operators $\phi_{l}(a)$. Instead we show that it is possible to introduce a family of Dirac operators $D_{a, \lambda}(m)$ whose domain incorporates functions with "specified branching" $e^{2 \pi i \lambda}$ at the points $a_{j}$ (our parametrization of the monodromy by $\lambda_{j}$ rather than the $l_{j}$ used by SMJ may seem a small point but it is ultimately the source of the difference between the results we present here and the SMJ results!). We show that it is possible to "localize" the differential operator $D_{a, \lambda}(m)$ away from its singularities (which consist of branch cuts emerging from the points $a_{j}$ ) and that the localization is completely characterized by a family of subspaces (see E. Witten [18] for a discussion of this idea) that belong to an infinite dimensional Grassmannian. There is a holomorphic line bundle, $\operatorname{det}^{*}$, over this Grassmannian first introduced by G. Segal and G. Wilson [15] and in $\S 4$ we show that there is a trivialization of this line bundle over the localization subspaces which makes mathematical sense of the following definition of $\tau(a)$ (see (4.9)):

$$
\tau(a)=\operatorname{det}\left(D_{a, \lambda}(m)\right) .
$$


We show that the logarithmic derivative of this $\tau$-function is computable in terms of data associated with the Green function $G^{a, \lambda}\left(z, z^{\prime}\right)$ for $D_{a, \lambda}(m)$. The fundamental result (see (3.62) and (3.63)) that connects this with the deformation theory is that $d_{a} G^{a, \lambda}\left(z, z^{\prime}\right)$ is finite rank and expressible in terms of the basic wave functions (which are not always in $L^{2}$ in our version-half of them are always in $L^{2}$ in the SMJ theory). It is interesting to note that the factorization property of the derivative of the Green function is a property that was emphasized by J. Myers in work on scattering theory that was the source of the Painleve functions in the WMTB paper [18]. As another illustration of the central character of the derivative $d_{a} G^{a, \lambda}$ we show in $\S 5$ that the deformation equations are an expression of the information in $d_{a}^{2} G^{a, \lambda}=0$.

There are, I believe, a number of advantages to defining the $\tau$ function as the determinant of a differential operator. The first is that the conceptual connection between the various elements of the SMJ analysis become transparent-given that the derivative of the Green function is expressed in terms of wave functions it is not surprising that the logarithmic derivative of the $\tau$-function can be expressed in terms of the local expansion coefficients for the wave functions. Secondly, the generalization of the SMJ theory to say, monodromy preserving deformations of the Cauchy-Riemann equations, is not precisely parallel to the Dirac case when one regards the deformation theory as fundamental. In contrast, the introduction of Cauchy-Riemann operators, $\bar{\partial}_{a, L}$ with domains that incorporate functions with prescribed branching (or monodromy) matrices, $e^{2 \pi i L_{J}}$, can be done in a fashion that precisely parallels the Dirac case [8]. In the regular singular case the $\tau$-function has been defined by Malgrange [4] in a fashion that makes it possible to write (see [8]):

$$
\tau(a)=\operatorname{det}\left(\bar{\partial}_{a, L}\right) \text {. }
$$

On the technical side the formula (4.9) for the $\tau$-function can also be evaluated in the transfer matrix formalism (this is done here at the end of $\S 4$ ). The result is a formula, (4.17), which can be matched with the formula for the scaling limit of the lattice $\tau$-function found in [7]. We are thus able to by-pass the auxiliary constructions in [3] in making a connection with the lattice theory. Osterwalder-Schrader. positivity is an immediate consequence of the control of this scaling limit and I believe that the rest of the Osterwalder-Schrader axioms can be confirmed for the appropriate subclass of $\tau$-functions (although this is not taken up here). 
Finally generalizations beyond the examples considered by SMJ are more easily approached through the formalism of differential operators and determinant bundles than they are through the representation theoretic formalism of free Fermi and Bose fields. The two formulations are related (by G. Segal's infinite dimensional version of the Borel-Weil construction [11]) but the less refined structures in the determinant bundle approach make it possible to ignore complications such as the "unitary" structure in the representation theoretic approach and to concentrate on the geometric role of the Green function in describing localizations. Generalizations to compact Riemann surfaces with genus greater than 0 for the Cauchy-Riemann theory and to manifolds with non-Euclidean metrics for Dirac theory immediately suggest themselves. I should mention that quite independent of these developments, C. Tracy has already introduced an extension of the deformation theory to the hyperbolic plane [15], [10] and in collaboration with R. Narayanan has results for the zero curvature limit of $\tau$-functions [6].

Incidentally, if one regards the operators $\bar{\partial}_{a, L}$ and $D_{a, \lambda}(m)$ as fundamental one sees that the Cauchy-Riemann theory naturally belongs on a compact Riemann surface and the Dirac theory needs the mass term to give a regular theory on $\mathbf{R}^{2}$. The reason is that it is important for the operators $\bar{\partial}_{a, L}$ and $D_{a, \lambda}(m)$ to be Fredholm operators. Compactness ensures that the Cauchy-Riemann operator will be Fredholm and the mass term makes the Dirac operator invertible. The Ising case (monodromy -1) is singular from this point of view as the associated Dirac operator $D_{a, \lambda}(m)$ is not Fredholm even with the mass term. One symptom of this is that there is more than one continuum theory associated with the monodromy parameter -1 (the scaling limits from above and below the critical point are different for example).

I would now like to describe the organization of this paper. The first section introduces the operator $D_{a, \lambda}(m)$ and summarizes a number of results from SMJ III about local expansions of multivalued solutions to the Dirac equation that will be used frequently in what follows. The reader should be aware that our parametrization of the local expansions differs from that in SMJ III. Some formulas, such as the local expansion formulas for the basic wave functions (see (5.3)) "look" the same as the formulas for the local expansions for the canonical wave functions in SMJ III but the two wave functions are different in general. 
The second section of the paper is devoted to the characterization of the $n$-point Green function for $D_{a, \lambda}(m)$ following some ideas from SMJ III and to the calculation of an explicit formula for the "onepoint" Green function. The principal results are (2.13), (2.14), (2.25) and (2.26). I believe these results are new but they are closely related to some formulas in SMJ IV which arise in calculating the normal ordered exponential representation for the holonomy fields $\phi_{l}(a)$.

The third section is devoted to an existence result for the $n$-point Green function, $G^{a, \lambda}\left(z, z^{\prime}\right)$, and to the calculation of its derivative in the ' $a$ ' variables. The calculation of this derivative again follows some ideas in SMJ III. The principal results are (3.62) and (3.63). SMJ introduce and deal with a Green function in SMJ III whose existence can be established by first constructing the Green function for the Helmholtz operator and then differentiating it to get the Green function for the Dirac operator. Our Green function differs from the SMJ Green function by a "wave function" whose existence can be inferred from the developments found in $\S 5$ of this paper. An existence proof for the Green function along these lines is simpler than the proof we have given but we did not present this proof for a number of reasons. The proof of the existence of the Green function that is given here is based on subspace localization ideas, transfer matrix calculations, and Fredholm theory. An advantage to this somewhat awkward combination is that it makes the connection with lattice results straightforward. The explicit calculation of the one-point Green function and the transfer matrix subspace transversality computation in $\S 3$ are both important in this regard, and the latter is, in any case, central to the $\tau$-function construction in $\S 4$.

The fourth section introduces the $\tau$-function. First a heuristic calculation of $\operatorname{det}\left(D_{a, \lambda}(m)\right)$ is made and then a rigorous definition (see (4.9)) is given and shown to connect with the result of this calculation. The principal result is Theorem 4.3.

The fifth section deduces the deformation equations for the low order expansion coefficients of our fundamental wave functions from the equation $d_{a}^{2} G^{a, \lambda}=0$. This section was included because, although it is possible to connect the expansion coefficients that appear in our formula for the logarithmic derivative of the $\tau$-function with the local expansion coefficients for the canonical wave functions of SMJ III, it is excessively awkward to do so. Our conclusion is that the deformation equations (5.21) and (5.22) with the algebraic side conditions (5.23) and (5.24) have exactly the same form as the SMJ III deformation 
equations with the single exception of a positive definiteness condition which is no longer satisfied by one of the matrices. We conclude by carrying out the analysis of the two-point case in the one circumstance where the result will look different from the SMJ analysis. It does not look very different.

I would like to express my gratitude to D. Quillen, G. Segal, E. Witten, and B. Malgrange whose clear ideas on the subject of determinant bundles and $\tau$-functions made this work possible. I must also express my debt to M. Sato, T. Miwa, and M. Jimbo. I have been studying their work for more than ten years now and I am still amazed by their achievement. Finally, I would like to thank C. Tracy for constant support and enthusiasm in the pursuit of what must seem an endless project.

\section{1. $D_{a, \lambda}(m)$ and local expansions.}

Incorporating branching in the domain of the Dirac operator. The Dirac operator in the plane is:

$$
D:=\left(\begin{array}{cc}
0 & -\partial_{z} \\
-\bar{\partial}_{z} & 0
\end{array}\right)
$$

where

$$
\partial_{z}=\frac{1}{2}\left(\partial_{x}-i \partial_{y}\right) \text { and } \bar{\partial}_{z}=\frac{1}{2}\left(\partial_{x}+i \partial_{y}\right)
$$

We are interested in defining a modified Dirac operator whose domain incorporates functions with specified branching at a collection of points $\left\{a_{1}, \ldots, a_{n}\right\}$ in $\mathbf{R}^{2}$ which we identify with $\mathbf{C}$ in the usual fashion. For simplicity we will suppose that all the points $a_{J}$ lie strictly in the lower half plane (that is, all the $a_{j}$ have second coordinates that are strictly less than 0$)$. Now choose real numbers $\lambda_{j}$ for $j=1, \ldots, n$ so that:

$$
-\frac{1}{2}<\lambda_{j}<\frac{1}{2}
$$

and define:

$$
y_{j}(z):=\left(z-a_{j}\right)^{\lambda_{\jmath}}=e^{\lambda_{\jmath} \log \left(z-a_{\jmath}\right)}
$$

where the logarithm in the definition is the principal value with branch cut on the positive real axis. As $z$ makes a counterclockwise circuit of $a_{j}$ the function $y_{j}$ changes by the monodromy factor $\Lambda_{j}:=e^{2 \pi i \lambda_{1}}$. We will say that the choice of $\lambda_{j}$ less than one half in absolute value realizes the monodromy $\Lambda_{j}$ for the function $y_{j}$ with "minimal singularity" at $a_{j}$ (the function $y_{j}$ and its inverse $y_{j}^{-1}$ are as "close" to the constant 1 as they can be). 
Let $\lambda=\sum_{j} \lambda_{j}$ and choose some point $b$ which is strictly in the upper half plane. Define:

$$
Y(z):=\frac{\prod_{j} y_{j}(z)}{(z-b)^{\lambda}}
$$

with the conventions regarding the logarithm in the definition of $(z-b)^{\lambda}$ as above. The function $Y(z)$ is holomorphic in the lower half plane except for branch cuts emanating from the branch points $a_{J}$ where it realizes the monodromy $\Lambda_{j}$ at $a_{j}$ with minimal singularity. The function $Y(z)$ tends to 1 near infinity on the negative real axis and tends to the constant $\prod \Lambda_{j}$ near infinity on the positive real axis. Between the branch cuts in the lower half plane the function $Y(z)$ tends to a constant that depends on the branch cuts involved. The behavior of $Y(z)$ in the upper half plane will not be of any consequence for us. We are now prepared to define a Dirac operator whose domain incorporates branching with monodromy $\Lambda_{j}$ at $a_{j}$. Let $\varphi$ denote a bounded $C^{\infty}$ function on $\mathbf{R}^{2}$ which is identically 1 in the lower half plane and which vanishes identically for arguments with second coordinates greater than some number slightly smaller than the second coordinate of $b$. Thus $\varphi Y$ is smooth in the upper half plane. Define:

$$
M(z):=\left(\begin{array}{cc}
Y(z) & 0 \\
0 & \bar{Y}(z)^{-1}
\end{array}\right) .
$$

A measurable function $f(z)$ will be in the domain of the operator we are interested in provided $\varphi M f \in H^{1}\left(\mathbf{R}^{2}\right)$ and $(1-\varphi) f \in H^{1}\left(\mathbf{R}^{2}\right)$ where $H^{1}$ is the standard Sobolev space of square integrable functions with square integrable first derivatives. We will denote this domain by $\mathscr{D}_{a, \lambda}$ and we observe that it has a norm given by:

$$
\|M \varphi f\|_{H^{1}}+\|(1-\varphi) f\|_{H^{1}} .
$$

It is not hard to check that this domain does not depend on the choice of $b$ or the choice of $\varphi$ although the norm does depend on these choices. If $f \in \mathscr{D}_{a, \lambda}$ we write:

$$
D_{a, \lambda} f:=M^{*} D M \varphi f+D(1-\varphi) f
$$

where $M^{*}$ denotes the transpose conjugate of the matrix $M$. A simple calculation shows that:

$$
M^{*} D M=\left(\begin{array}{cc}
0 & -\bar{Y} \partial_{z} \bar{Y}^{-1} \\
-Y^{-1} \bar{\partial}_{z} Y & 0
\end{array}\right) .
$$

Since $Y$ is holomorphic and $\bar{Y}$ is anti-holomorphic in a neighborhood of the real axis it follows that $\partial_{z}(\bar{Y})$ and $\bar{\partial}_{z}(Y)$ both vanish in a 
neighborhood of the real axis. Thus the action of $M^{*} D M$ is the same as the action of $D$ for functions with support in a neighborhood of the real axis. From this one may easily deduce that the operator $D_{a, \lambda}$ does not depend on the choice of $\varphi$. Similar considerations show that $D_{a, \lambda}$ does not depend on the choice of $b$ either. It is clear that when $D_{a, \lambda}$ is applied to a function $f \in \mathscr{D}_{a, \lambda}$ that the result lands in:

$$
\mathscr{R}_{a, \lambda}:=M^{*} L^{2}\left(\mathbf{R}_{-}^{2}\right) \oplus L^{2}\left(\mathbf{R}_{+}^{2}\right)
$$

where $\mathbf{R}_{-}^{2}$ and $\mathbf{R}_{+}^{2}$ are the lower and upper half planes respectively. The range space $\mathscr{R}_{a, \lambda}$ has a norm given by:

$$
\left\|M^{*-1} g\right\|_{L_{-}^{2}}+\|g\|_{L_{+}^{2}}
$$

Now choose $m>0$. We are now able to define the "massive" Dirac operator which will be the principal object of our study:

$$
D_{a, \lambda}(m)=D_{a, \lambda}+m I
$$

where $I$ is the $2 \times 2$ identity matrix. To make simple sense of this as a map from $\mathscr{D}_{a, \lambda}$ to $\mathscr{R}_{a, \lambda}$ we will show that:

$$
\mathscr{D}_{a, \lambda} \subset \mathscr{R}_{a, \lambda} \text {. }
$$

Suppose that $f \in \mathscr{D}_{a, \lambda}$. We want to show that $f \in \mathscr{R}_{a, \lambda}$. It is clear that $f$ is in $H^{1}\left(\mathbf{R}_{+}^{2}\right)$ and so it is automatically square integrable in the upper half plane. It remains to check that $M^{*-1} f$ is square integrable in the lower half plane. But $M^{*-1} f=\left(M M^{*}\right)^{-1} M f$ and $M f$ is in $H^{1}$ of the lower half plane. Let $D_{j}$ denote a small disk about the point $a_{j}$. In the complement of the union of the disks $D_{j}$ in the lower half plane the matrix function $\left(M M^{*}\right)^{-1}$ is uniformly bounded. Thus $M^{*-1} f$ is square integrable in this complement. Employing a smooth partition of unity to localize $f$ inside the disks $D_{j}$ one sees that it will suffice to show that if $g$ is an $H^{1}$ function with support in some $D_{j}$ then $\left(M M^{*}\right)^{-1} g$ will be in $L^{2}$. The relevant singularity in $\left(M M^{*}\right)^{-1}$ near $a_{j}$ comes from the factor $\left|z-a_{j}\right|^{ \pm 2 \lambda}{ }_{j}$. It will suffice to show that if $g$ is a function which is in $H^{1}$ in a neighborhood of a point (which we may take to be 0 for simplicity) and $\alpha<1$ is a real number then $r^{-\alpha} g$ is locally square integrable, where $r$ is the distance from 0 . It is not hard to see that the $L^{2}$ norm of the radial derivative of $g$ will be finite if $g \in H^{1}$. The reader will not have any trouble using this in turn to check that the desired result is implied by the following proposition (in which the constant $\beta=2 \alpha-1$ ): 
Proposition 1.0. Suppose that $g(r)$ is a smooth function of $r$ with support in the domain $0 \leq r<1$. Let $\beta$ be a real number strictly less than 1. Then:

$$
\int_{0}^{1} r^{-\beta}|g(r)|^{2} d r \leq\left(\frac{2}{1-\beta}\right)^{2} \int_{0}^{1}\left|g^{\prime}(r)\right|^{2} r d r .
$$

Proof. It will suffice to prove this when $g$ is real. Calculating a derivative in $r$ one finds:

$$
\frac{d}{d r}\left(r^{1-\beta} g(r)^{2}\right)=(1-\beta) r^{-\beta} g(r)^{2}+2 r^{1-\beta} g(r) g^{\prime}(r) .
$$

Integrating this from 0 to 1 and using the fact that $r^{1-\beta}$ vanishes at 0 and $g(r)$ vanishes at $r=1$ it follows that:

$$
(1-\beta) \int_{0}^{1} r^{-\beta} g(r)^{2} d r=-2 \int_{0}^{1} r^{1-\beta} g(r) g^{\prime}(r) d r .
$$

The Schwartz inequality implies:

$$
\int_{0}^{1} r^{-\beta} g(r)^{2} d r \leq \frac{2}{1-\beta}\left(\int_{0}^{1} g^{\prime}(r)^{2} r d r\right)^{1 / 2}\left(\int_{0}^{1} r^{1-2 \beta} g(r)^{2} d r\right)^{1 / 2} \text {. }
$$

Since $2 a b \leq \varepsilon^{-1} a^{2}+\varepsilon b^{2}$ for any $\varepsilon>0$ and $r^{1-2 \beta} \leq r^{-\beta}$ for $0 \leq r \leq 1$ it follows that the right-hand side of this last inequality is dominated by:

$$
\frac{\varepsilon^{-1}}{1-\beta} \int_{0}^{1} g^{\prime}(r)^{2} d r+\frac{\varepsilon}{1-\beta} \int_{0}^{1} r^{-\beta} g(r)^{2} d r .
$$

If we now choose $\varepsilon=\frac{1-\beta}{2}$ in this last expression and combine this with the previous inequality then the desired result follows.

Local expansions. We will understand the singular operator $D_{a, \lambda}(m)$ primarily by localizing it away from its singularities (at the points $\left.a_{1}, \ldots, a_{n}\right)$. In order to formulate and to understand these localizations we will make heavy use of results for local expansions of multivalued solutions to the Dirac equation. Suppose that $a$ is a point in the plane and that $B$ is an open ball centered at $a$. Let $\widetilde{B}$ denote the simply connected covering of $B \backslash\{a\}$. Suppose that $p \in B$ is a point different from $a$ and let $\gamma$ denote a simple closed curve which starts at $p$ and makes a counterclockwise circuit of $a$. Then $\gamma$ generates the fundamental group of $B \backslash\{a\}$ and it acts as a deck transformation on $\widetilde{B}$. We will say that $\tilde{w}: \widetilde{B} \rightarrow \mathbf{C}^{2}$ is a multivalued solution to the Dirac equation with monodromy $\Lambda$ at $a$ if:

$$
D(m) \tilde{w}(x)=0 \quad \text { for } x \in \widetilde{B}
$$


and

$$
\tilde{w}(\gamma x)=\Lambda \tilde{w}(x) \text { for } x \in \widetilde{B} .
$$

Now let $b$ denote the horizontal ray with vertex $a$ which emerges to the right of $a$. If $w(x)$ is a solution to the Dirac equation on the open set $B \backslash b$, and $w(x)$ is a branch of a multivalued solution $\tilde{w}(x)$ with monodromy $\Lambda$ at $a$ then we will say that $w(x)$ is solution to the Dirac equation with monodromy $\Lambda$ near $a$ which is branched along $b$. What is at issue here is that away from the vertex $a$ along the branch cut $b$, the function $w(x)$ must have boundary values that continue smoothly across $b$ as solutions to the Dirac equation and that the upper and lower continuations differ by a factor of $\Lambda$. It is a result of SMJ III that such solutions to the Dirac equation are characterized in terms of local expansions. We will now summarize the results concerning these local expansions that will be of use to us. Let $R$ denote the differential operator:

$$
R:=z \partial_{z}-\bar{z} \bar{\partial}_{z}+\frac{1}{2}\left[\begin{array}{cc}
1 & 0 \\
0 & -1
\end{array}\right] .
$$

It is easy to check that this "infinitesimal rotation" commutes with the Dirac operator $D(m)$. Now let $l$ denote a real number and consider the differential equations:

$$
D(m) \psi=0, \quad R \psi=l \psi
$$

where $\psi$ is a $\mathbf{C}^{2}$ valued function on the simply connected covering space of $\mathbf{R}^{2} \backslash\{0\}$. It is not hard to see that the "global" consequence of being an eigenvector for $R$ with eigenvalue $l$ is that $\psi(x)$ changes by a factor of $e^{2 \pi i(l+1 / 2)}$ as $x$ makes a counterclockwise circuit of 0 . By working in polar coordinates it is not hard to see that the space of such solutions $\psi$ is two dimensional. Furthermore by calculating the commutator of $R$ with $\partial_{z}$ and $\bar{\partial}_{z}$ one sees that the latter two differential operators will map the $l$ eigenspace for $R$ into the $l-1$ and $l+1$ eigenspaces for $R$ respectively. Following SMJ III we now define:

$$
w_{l}(r, \theta)=\left(\begin{array}{l}
e^{i(l-1 / 2) \theta} I_{l-1 / 2}(m r) \\
e^{i(l+1 / 2) \theta} I_{l+1 / 2}(m r)
\end{array}\right)
$$

and

$$
w_{l}^{*}(r, \theta)=\left(\begin{array}{l}
e^{-i(l+1 / 2) \theta} I_{l+1 / 2}(m r) \\
e^{-i(l-1 / 2) \theta} I_{l-1 / 2}(m r)
\end{array}\right)
$$


where $I_{l}(r)$ is the modified Bessel function of the first kind. The functions $w_{l}$ and $w_{l}^{*}$ determine multivalued solutions to the Dirac equation $D(m) w=0$ and furthermore:

$$
R w_{l}=l w_{l}, \quad R w_{l}^{*}=-l w_{l}^{*} .
$$

The functions $w_{l}$ and $w_{-l}^{*}$ span the two dimensional space of (multivalued) solutions to the Dirac equation which are also eigenfunctions of the infinitesimal rotation $R$ with eigenvalue $l$. These eigenfunctions are singled out by the requirement that the other infinitesimal symmetries of the Dirac operator, $\partial_{z}$ and $\bar{\partial}_{z}$ act in a simple fashion as "ladder" operators. In fact, one has:

$$
\partial w_{l}=\frac{m}{2} w_{l-1}, \quad \bar{\partial} w_{l}=\frac{m}{2} w_{l+1}
$$

and

$$
\partial w_{l}^{*}=\frac{m}{2} w_{l+1}^{*}, \quad \bar{\partial} w_{l}^{*}=\frac{m}{2} w_{l-1}^{*} .
$$

Finally, the parametrization of $w_{l}^{*}$ by minus the eigenvalue of $R$ rather than the eigenvalue itself is done so that both $w_{l}$ and $w_{-l}^{*}$ become locally less singular at 0 at $l$ increases. This simple change will be very useful in sorting out the local singularity structure of multivalued solutions to the Dirac equation-something that will be important for us in what follows.

It is a result of SMJ III that any multivalued solution of the Dirac equation, $w$, defined near 0 and with monodromy $\Lambda=e^{2 \pi i \lambda}$ at 0 has a local expansion:

$$
w(r, \theta)=\sum_{k}\left\{a_{k}(w) w_{k+\lambda}(r, \theta)+b_{k}(w) w_{k-\lambda}^{*}(r, \theta)\right\}
$$

where the sum is over the half-integers $\mathbf{Z}+\frac{1}{2}$ and the coefficients $a_{k}(w)$ and $b_{k}(w)$ are complex numbers. For fixed $r$ this expansion can be identified with the Fourier series expansion for the smooth $2 \pi$ periodic function $\theta \rightarrow e^{-i \lambda \theta} w(r, \theta)$ and consequently the series converges rapidly at fixed $r$ (it certainly converges absolutely for example).

Proposition 1.1. Suppose that $|\lambda|<\frac{1}{2}$ and that $w(z)$ is a multivalued solution to the Dirac equation in a neighborhood of 0 with monodromy $e^{-2 \pi i \lambda}$. Then $w(z)$ is locally in the domain of $D_{0, \lambda}(m)$ if and only if it has a local expansion of the form:

$$
w=\sum_{k>0}\left\{a_{k}(w) w_{k-\lambda}+b_{k}(w) w_{k+\lambda}^{*}\right\}
$$

where the sum is now over the positive half-integers. 
Proof. Let

$$
M(z)=\left[\begin{array}{cc}
z^{\lambda} & 0 \\
0 & \bar{z}^{-\lambda}
\end{array}\right]
$$

with the principal values for the fractional powers as in the first section. To say that $w(z)$ is locally in $\mathscr{D}_{0, \lambda}$ means that for some $\delta>0$ the (single valued) function $M(z) w(z)$ is in Sobolev space $H^{1}\left(B_{\delta}\right)$ where $B_{\delta}$ is the ball of radius $\delta$ about 0 . We will first show that if $w$ has an expansion of the form (1.5) then $M(z) w(z)$ is in $H^{1}$ near 0 . Suppose then that $w$ is a multivalued solution to the Dirac equation with a local expansion (1.5). We know that for some $r_{0}>0$ the Fourier expansion of the single valued function $e^{i \lambda \theta} w\left(r_{0}, \theta\right)$ is absolutely convergent. Choose $\delta<r_{0}$. We will show that $M(z) w(z)$ is in $H^{1}\left(B_{\delta}\right)$. We begin by identifying the Fourier coefficients of $e^{i \lambda \theta} w(r, \theta)$. If

$$
e^{i \lambda \theta} w(r, \theta)=\sum_{n=-\infty}^{\infty} e^{i n \theta}\left[\begin{array}{l}
f_{n}(r) \\
g_{n}(r)
\end{array}\right]
$$

is the Fourier expansion then using (1.0) and (1.1) the Fourier coefficients $f_{n}(r)$ and $g_{n}(r)$ are given by:

$$
f_{n}(r)= \begin{cases}a_{n+1 / 2} I_{n-\lambda}(m r) & \text { for } n \geq 0, \\ b_{-n-1 / 2} I_{-n+\lambda}(m r) & \text { for } n<0\end{cases}
$$

and

$$
g_{n}(r)= \begin{cases}a_{n-1 / 2} I_{n-\lambda}(m r) & \text { for } n>0 \\ b_{-n+1 / 2} I_{-n+\lambda}(m r) & \text { for } n \leq 0\end{cases}
$$

where $n$ is an integer. The following formula for the modified Bessel functions will allow us to obtain simple estimates for the Fourier coefficients $f_{n}$ and $g_{n}$ :

$$
I_{\nu}(r)=\frac{r^{\nu}}{2^{\nu} \Gamma\left(\nu+\frac{1}{2}\right)} h_{\nu}(r)
$$

where $\operatorname{Re}(\nu)>-\frac{1}{2}$ and

$$
h_{\nu}(r)=\frac{2}{\sqrt{\pi}} \int_{0}^{1}\left(1-s^{2}\right)^{\nu-1 / 2} \cosh (r s) d s .
$$

It is clear from this last formula that $h_{\nu}(r)$ is an increasing function of $r$ and using this fact together with (1.6) and (1.7) one finds: 


$$
\left|f_{n}(r)\right| \leq \begin{cases}\left(\frac{r}{r_{0}}\right)^{n-\lambda}\left|f_{n}\left(r_{0}\right)\right| & \text { for } n \geq 0 \\ \left(\frac{r}{r_{0}}\right)^{-n+\lambda}\left|f_{n}\left(r_{0}\right)\right| & \text { for } n<0\end{cases}
$$

and

$$
\left|g_{n}(r)\right| \leq \begin{cases}\left(\frac{r}{r_{0}}\right)^{n-\lambda}\left|g_{n}\left(r_{0}\right)\right| & \text { for } n>0 \\ \left(\frac{r}{r_{0}}\right)^{-n+\lambda}\left|g_{n}\left(r_{0}\right)\right| & \text { for } n \leq 0\end{cases}
$$

where $r \leq r_{0}$. One sees from this that the Fourier coefficients tend at least geometrically to 0 as $n \rightarrow \infty$ for $r<r_{0}$. Now let $\psi(r, \theta)$ denote the function $M(z) w(z)$ considered as a function of $(r, \theta)$. Then one has:

$$
\psi(r, \theta)=\sum_{n=-\infty}^{\infty} e^{i n \theta}\left[\begin{array}{c}
r^{\lambda} f_{n}(r) \\
r^{-\lambda} g_{n}(r)
\end{array}\right]
$$

for the Fourier expansion of $\psi(r, \theta)$. To show that this function is in $H^{1}\left(B_{\delta}\right)$ one needs to show that $\psi, \frac{\partial \psi}{\partial r}$, and $\frac{1}{r} \frac{\partial \psi}{\partial \theta}$ are all in $L^{2}\left(B_{\delta}\right)$. Calculating the $L^{2}\left(B_{\delta}\right)$ norm for $\psi$ in polar coordinates and making use of the orthogonality of the functions $e^{i n \theta}$ on the circle one finds this $L^{2}$ norm is proportional to:

$$
\sum_{n=-\infty}^{\infty} \int_{0}^{\delta} r^{2 \lambda}\left|f_{n}(r)\right|^{2} r d r+\sum_{n=-\infty}^{\infty} \int_{0}^{\delta} r^{-2 \lambda}\left|g_{n}(r)\right|^{2} r d r
$$

Using the assumption $|\lambda|<\frac{1}{2}$ and the geometric estimate (1.8)-(1.9) for the Fourier coefficients $f_{n}(r)$ and $g_{n}(r)$ for $r<\delta<r_{0}$ one easily checks that this is finite. The $r$ dependence of $f_{n}(r)$ is given by $r^{n} h_{n-\lambda}(r)$ for $n \geq 0$ and by $r^{-n+2 \lambda} h_{-n+\lambda}(r)$ for $n<0$. In every case except $n=0$ one finds that differentiating the power terms with respect to $r$ brings down a factor $\frac{1}{r}$ and a multiplier roughly of size $|n|$. The reader should check that in each case (excluding, of course, $n=0$ ) the additional factor of $\frac{1}{r}$ gives terms in the analogue of (1.10) for $\frac{\partial \psi}{\partial r}$ that remain locally integrable. The additional factor of order $|n|$ is easily controlled by the geometric convergence (1.8)(1.9) and the terms in which $h_{n-\lambda}(r)$ and $h_{-n+\lambda}(r)$ are differentiated are estimated by the obvious inequality $h_{\nu}^{\prime}(r) \leq h_{\nu}(r)$. Something analogous occurs for the Fourier coefficients of $g_{n}^{\prime}(r)$ and this suffices 
to demonstrate that $\frac{\partial \psi}{\partial r}$ is in $L^{2}\left(B_{\delta}\right)$. Every Fourier coefficient of $\frac{1}{r} \frac{\partial \psi}{\partial \theta}$ picks up an additional local singularity of $\frac{1}{r}$ except the $n=0$ coefficient (since this is killed by $\partial / \partial \theta$ ) and an additional factor of order $|n|$ compared to the Fourier coefficients of $\psi$. Once again this suffices to show that the analogue of (1.10) for $\frac{1}{r} \frac{\partial \psi}{\partial \theta}$ is finite and hence that this function is in $L^{2}\left(B_{\delta}\right)$.

We leave it to the reader to check that $M w_{k-\lambda}$ and $M w_{k+\lambda}^{*}$ for $k<0$ are too locally singular at 0 to be in $H^{1}\left(B_{\delta}\right)$. Since $M w_{k-\lambda}$ and $M w_{k+\lambda}^{*}$ make direct contributions to the $H^{1}\left(B_{\delta}\right)$ norm through their Fourier coefficients it follows that no terms involving $w_{k-\lambda}$ or $w_{k+\lambda}^{*}$ can occur for $k<0$ in the local expansion of a function in $\mathscr{D}_{a, \lambda}$. This finishes the proof of the proposition.

We next recall Proposition 3.1.5 from SMJ III. Let $\hat{w}_{l}$ denote the function defined by:

$$
\hat{w}_{l}(z)=w_{l}^{*}(z)-w_{-l}(z) .
$$

Then it is a result of SMJ III that $\hat{w}_{l}$ is square integrable at $\infty$. Up to a constant multiple $\hat{w}_{l}$ is the only multivalued solution to the Dirac equation (branched at 0 ) which is an eigenfunction for $R$ with eigenvalue $-l$ and which is square integrable at $\infty$. It is a result of SMJ III that any multivalued solution to the Dirac equation which is square integrable in a neighborhood of $\infty$ and has monodromy multiplier $e^{2 \pi i \lambda}$ in making a counter-clockwise circuit of a sufficiently large circle about the origin has a convergent expansion:

$$
W(z)=\sum_{k \in \mathbf{Z}+1 / 2} c_{k}(W) \hat{w}_{k-\lambda}(z) .
$$

In SMJ III this result is used to prove the following proposition (see Proposition 3.1.5. in [14-III]).

Proposition 1.2. Suppose that $w$ is a multivalued solution to the Dirac equation which is branched at $a_{1}, \ldots, a_{n}$. If $w$ is square integrable in the exterior of the ball of radius $R$ about 0 then in a neigborhood of $z=\infty$ one has:

$$
|P w(z)|=O\left(\frac{e^{-m|z|}}{\sqrt{|z|}}\right)
$$

where $P$ is any polynomial in the differential operators $\partial_{z}, \bar{\partial}_{z}$, and $R$ with constant coefficients. Furthermore any multivalued solution of 
the Dirac equation with a local expansion (1.11) in a neighborhood of $\infty$ is square integrable near $\infty$.

This will be of use to us when we examine the properties of the Green function for $D_{a, \lambda}(m)$ in more detail. To conclude this section we formulate a uniqueness result that is a consequence of the formula 3.1.18 in SMJ III for the inner product of two (multivalued) $L^{2}$ solutions to the Dirac equations in terms of their local expansion coefficients. Because of the slight difference in our notation for the local expansion coefficients the result has a different look to it here. Suppose that $w$ and $u$ are two multivalued solutions to the Dirac equation which have the same (unitary) monodromy about the branch points $\left\{a_{1}, \ldots, a_{n}\right\}$. One finds then that:

$$
\begin{aligned}
\frac{i m}{2} w(z) \bar{u}(z) d z d \bar{z} & =d\left(w_{2}(z) \bar{u}_{1}(z) i d \bar{z}\right) \\
& =d\left(w_{1}(z) \bar{u}_{2}(z) i d z\right) .
\end{aligned}
$$

Suppose now that $w$ and $u$ are locally in $L^{2}$ at each of the branch points and globally in $L^{2}$ near $\infty$. Let $R(\varepsilon)$ denote the complement in $\mathbf{R}^{2}$ of the union of the disks of radius $\varepsilon$ about the branch points $a_{j}$ for $j=1, \ldots, n$. Let $C_{j}(\varepsilon)$ denote the circle of radius $\varepsilon$ about the point $a_{j}$. Then Stoke's theorem transforms the integral:

$$
\frac{i m}{2} \int_{R(\varepsilon)} w(z) \bar{u}(z) d z d \bar{z}
$$

into an integral over the union of the circles $C_{j}(\varepsilon)$. It is a simple matter to use local expansion results for $w$ and $u$ to evaluate those boundary integrals in the limit $\varepsilon \rightarrow 0$. Suppose that the infinitesimal exponent of monodromy for $w$ at $a_{j}$ is $\lambda_{j}$. Then $w$ and $u$ will have local expansions at $a_{\nu}$ of the following sort:

$$
w=\sum_{k}\left\{a_{k}^{\nu}(w) w_{k+\lambda_{\nu}}+b_{k}^{\nu}(w) w_{k-\lambda_{\nu}}^{*}\right\}
$$

where the sum is over the half-integers $\mathbf{Z}+\frac{1}{2}$ and we have written $w_{k+\lambda_{\nu}}$ and $w_{k-\lambda_{\nu}}^{*}$ as shorthand for $w_{k+\lambda_{\nu}}\left(z-a_{\nu}\right)$ and $w_{k-\lambda_{\nu}}^{*}\left(z-a_{\nu}\right)$. We now divide the integers from 1 to $n$ into two classes. Let $\mathbf{N}$ denote those elements $j \in\{1, \ldots, n\}$ such that $\lambda_{j}<0$ and let $\mathbf{P}$ denote those elements $j \in\{1, \ldots, n\}$ such that $\lambda_{j}>0$. Recalling that $\left|\lambda_{j}\right|<\frac{1}{2}$ it is easy to check the smallest index $k$ for which $w_{k+\lambda_{v}}$ 
is in $L^{2}$ at $a_{\nu}$ is $-1 / 2$ if $\nu \in \mathbf{P}$ and $1 / 2$ if $\nu \in \mathbf{N}$. Thus we define:

$$
c_{\nu}(w)= \begin{cases}a_{-1 / 2}^{\nu}(w), & \nu \in \mathbf{P}, \\ a_{1 / 2}^{\nu}(w), & \nu \in \mathbf{N} .\end{cases}
$$

In a similar fashion one sees that the smallest value of $k$ for which $w_{k-\lambda_{\nu}}^{*}$ is in $L^{2}$ near $a_{\nu}$ is $-1 / 2$ for $\nu \in \mathbf{N}$ and $1 / 2$ for $\nu \in \mathbf{P}$. Thus we define:

$$
c_{\nu}^{*}(w)= \begin{cases}b_{-1 / 2}^{\nu}(w), & \nu \in \mathbf{N}, \\ b_{1 / 2}^{\nu}(w), & \nu \in \mathbf{P} .\end{cases}
$$

Using (1.0) and (1.1) to do the Stokes' theorem calculation(s) suggested above one finds:

Proposition 1.3. Suppose that $w$ and $u$ are two multivalued solutions to the Dirac equation both branched at the points $\left\{a_{1}, \ldots, a_{n}\right\}$ with the same unitary monodromy. If both solutions are in $L^{2}\left(\mathbf{R}^{2}\right)$ then:

$$
\frac{m}{2} \int_{\mathbf{R}^{2}} w(z) \bar{u}(z) i d z d \bar{z}=-\frac{4}{m} \sum_{\nu}\left|s_{\nu}\right| c_{\nu}(w) \overline{c_{\nu}^{*}(u)}
$$

and

$$
\frac{m}{2} \int_{\mathbf{R}^{2}} w(z) \bar{u}(z) i d z d \bar{z}=-\frac{4}{m} \sum_{\nu}\left|s_{\nu}\right| c_{\nu}^{*}(w) \overline{c_{\nu}(u)}
$$

where:

$$
s_{\nu}:=\sin \left(\pi \lambda_{\nu}\right)
$$

and the two different forms result from the two different ways in which the left-hand side of (1.12) is expressed as a derivative.

The uniqueness result we want is a direct consequence of Proposition 1.3.

Proposition 1.4. Suppose that $w$ is a multivalued solution to the Dirac equation which is square integrable at infinity and which has local expansion:

$$
w=\sum_{k>0}\left\{a_{k}^{\nu} w_{k+\lambda_{\nu}}+b_{k}^{\nu} w_{k-\lambda_{\nu}}^{*}\right\}
$$

at the branch point $a_{\nu}$. Then the function $w$ is identically zero.

Proof. To see this one needs only calculate the $L^{2}$ norm of $w$ using (1.13) and:

$$
c_{\nu}(w)= \begin{cases}0, & \nu \in \mathbf{P} \\ a_{1 / 2}^{\nu}(w), & \nu \in \mathbf{N}\end{cases}
$$




$$
c_{\nu}^{*}(w)= \begin{cases}b_{1 / 2}^{\nu}(w), & \nu \in \mathbf{P}, \\ 0, & \nu \in \mathbf{N} .\end{cases}
$$

2. Calculating the Green function for $D_{a, \lambda}$ when $n=1$.

Green functions. In this section we will define the Green function for the operator $D_{a, \lambda}(m)$ and examine some of its properties assuming that it exists. In succeeding sections we will prove the existence of the Green function-first for the case of just one branch point by exhibiting a formula for the Green function and then in the case of $n$ branch points using a combination of transfer matrix ideas, localization, and Fredholm theory together with the formula from the one point case. The Green function we construct will allow us to solve the equation:

$$
D_{a, \lambda}(m) f=g
$$

given a function $g$ in $L^{2}$ which has support disjoint from $b$, the union of the branch cuts $b_{j}$ emanating to the right from the branch points $a_{j}$ for $j=1, \ldots, n$. Our solution will end up in the domain $\mathscr{D}_{a, \lambda}$ but we will not attempt to prove the estimates needed to extend this to a full inverse on all of $\mathscr{R}_{a, \lambda}$. It is useful to begin with a calculation of the Green function for $D(m)$ where no branch points are involved.

The Green function for $D(m)$ on $\mathbf{R}^{2}$. Consider the Euclidean Dirac operator defined on $\mathbf{R}^{2}$ by:

$$
D:=\left(\begin{array}{cc}
0 & -\partial_{z} \\
-\bar{\partial}_{z} & 0
\end{array}\right)
$$

where $\partial_{z}=\frac{1}{2}\left(\partial_{x}-i \partial y\right)$. We are interested in the Dirac operator with a mass term defined by:

$$
D(m)=D+\frac{m}{2} I
$$

where $I$ is the $2 \times 2$ identity operator. In the Fourier transform variables

$$
\hat{f}(\xi)=\frac{1}{4 \pi^{2}} \int_{\mathbf{R}^{2}} f(x) e^{-l x \cdot \xi} d x
$$

the Dirac operator becomes multiplication by the matrix:

$$
\frac{1}{2}\left(\begin{array}{cc}
m & -i \bar{\xi} \\
-i \xi & m
\end{array}\right)
$$


where $\xi=\xi_{1}+i \xi_{2}$. The Green function, $G_{0}(x)$, for the Dirac operator is thus:

$$
G_{0}(x)=\frac{1}{2 \pi^{2}} \int_{\mathbf{R}^{2}}\left(\begin{array}{cc}
m & i \bar{\xi} \\
i \xi & m
\end{array}\right) \frac{e^{i x \cdot \xi}}{|\xi|^{2}+m^{2}} d \xi
$$

In a later calculation it will be very convenient to identify a particular contour integral representation for this Green function. Suppose that $x_{2}>0$. Then the $d \xi_{2}$ integral can be closed in the upper half plane with a single contribution from the pole at $\xi_{2}=i \sqrt{\xi_{1}^{2}+m^{2}}$. One finds:

$$
G_{0}(x)=\frac{1}{2 \pi} \int_{\mathbf{R}}\left(\begin{array}{cc}
m & i \xi_{+} \\
i \xi_{-} & m
\end{array}\right) \frac{e^{(i / 2)\left(x \xi_{+}+\bar{x} \xi_{-}\right)}}{\sqrt{\xi_{1}^{2}+m^{2}}} d \xi_{1}
$$

where $\xi_{ \pm}:=\xi_{1} \pm \sqrt{\xi_{1}^{2}+m^{2}}$. Now introduce a new variable $u$ defined by:

then:

$$
u=-\frac{i}{m} \xi_{-}
$$

and one finds:

$$
u^{-1}=-\frac{i}{m} \xi_{+}
$$

$$
G_{0}(x)=m \int_{i \mathbf{R}^{+}}\left(\begin{array}{cc}
1 & -u^{-1} \\
-u & 1
\end{array}\right) e^{-(m / 2)\left(\bar{x} u+x u^{-1}\right)} \frac{d u}{2 \pi u}
$$

where $i \mathbf{R}^{+}:=\left\{u \mid u=i \alpha\right.$ with $\left.\alpha \in \mathbf{R}^{+}\right\}$. The exponential factor in this last integrand decays as $u$ tends to 0 and also as $u$ tends to $\infty$. In fact, it is easy to see that Cauchy's theorem permits one to deform the contour $i \mathbf{R}^{+}$to any other ray $b \mathbf{R}^{+}$with $\operatorname{Re}(\bar{x} b)=x \cdot b>0$ (indeed, our choice of the variable $u$ was determined by the desire that the admissible rays, $b \mathbf{R}^{+}$, should have such a simple direct connection to the disposition of the point $x$ ).

In particular one can choose $b=x$. Thus:

$$
G_{0}(x)=m \int_{x \mathbf{R}^{+}}\left(\begin{array}{cc}
1 & -u^{-1} \\
-u & 1
\end{array}\right) e^{-(m / 2)\left(\bar{x} u+x u^{-1}\right)} \frac{d u}{2 \pi u} .
$$

We leave it to the reader to check that this representation of the Green function is valid even without the restriction $x_{2}>0$. We may also use the integral representations to identify the matrix elements of $G_{0}(x)$ with modified Bessel functions. One finds:

$$
G_{0}(x)=\frac{m}{\pi}\left(\begin{array}{cc}
K_{0}(m|x|) & \frac{\bar{x}}{|x|} K_{0}^{\prime}(m|x|) \\
\frac{x}{|x|} K_{0}^{\prime}(m|x|) & K_{0}(m|x|)
\end{array}\right)
$$

where as before $x=x_{1}+i x_{2}$. 
It will be very convenient for later developments to make changes in the kernel $G_{0}(x)$ associated with using the measure $i d x d \bar{x}=$ $2 d x_{1} d x_{2}$ and the bilinear form $(x, y):=x \cdot J y$ where:

$$
J:=\left(\begin{array}{cc}
0 & i \\
-i & 0
\end{array}\right)
$$

Define:

$$
G(x):=\frac{1}{2} G_{0}(x) J
$$

so that the solution of $D(m) f=g$ (for $g \in L^{2}\left(\mathbf{R}^{2}\right)$ ) is given by:

$$
f(x)=\int_{\mathbf{R}^{2}} G(x-y) J g(y) i d y d \bar{y} .
$$

One can express this same observation as:

$$
\int_{\mathbf{R}^{2}}\left(G_{i,} \cdot(x-y), D(m) f(y)\right) i d y d \bar{y}=f_{i}(x)
$$

where $G_{i},(x)$ denotes the $i$ th row of $G(x)$ (regarded as a vector in $\left.\mathbf{C}^{2}\right)$. The reason we make this change is that the operator $D(m)$ is formally symmetric with respect to the bilinear form $(\cdot, \cdot)$. One consequence of this is that not only are the columns of $G(x)$ solutions to the Dirac equation but the rows are as well. In the calculation of the more complicated Green function for $D_{a, \lambda}(m)$ the use of this bilinear pairing will allow us to work exclusively with the Dirac equation (were we to use the standard Euclidean inner product we would be forced to deal with solutions to the adjoint equation $D(m)^{*} \psi=0$ as well).

Defining the Green function for $D_{a, \lambda}(m)$. We will now define the Green function for $D_{a, \lambda}(m)$. The Green function $G^{a, \lambda}\left(z, z^{\prime}\right)$ will denote the kernel of the inverse for $D_{a, \lambda}(m)$. If

$$
D_{a, \lambda} f(z)=g(z)
$$

then we want to recover $f$ from $g$ as follows:

$$
f(z)=\int_{\mathbf{R}^{2} \backslash b} G^{a, \lambda}\left(z, z^{\prime}\right) J g\left(z^{\prime}\right) i d z^{\prime} d \bar{z}^{\prime}
$$

where the essential support of $g$ is supposed to be disjoint from $b$, the union of the branch cuts $b_{j}$ for $j=1, \ldots, n$. In order that $f(z)$ in (2.4) should satisfy the Dirac equation we want:

G1: The columns of the map $z \rightarrow G^{a, \lambda}\left(z, z^{\prime}\right)$ are multivalued solutions of the Dirac equation, defined for $z \in \mathbf{R}^{2} \backslash\left\{z^{\prime}\right\} \cup b$, and 
branched along $b_{j}$ with monodromy $\Lambda_{j}=e^{-2 \pi i \lambda}$. These solutions have local expansions near $a_{\nu}$ given by:

$$
\begin{aligned}
& G_{\cdot, j}^{a, \lambda}\left(z, z^{\prime}\right) \\
& \quad=\sum_{k>0}\left\{a_{k, j}^{\nu}\left(z^{\prime}\right) w_{k-\lambda_{\nu}}\left(z-a_{\nu}\right)+b_{k, j}^{\nu}\left(z^{\prime}\right) w_{k+\lambda_{\nu}}^{*}\left(z-a_{\nu}\right)\right\}
\end{aligned}
$$

where the notation $G_{., j}$ signifies the $j$ th column vector of the matrix $G$.

The restriction on the local expansions in (2.5) is suggested by Proposition (1.1) and will insure that the function $f$ is locally in $\mathscr{D}_{a, \lambda}$. To insure that $f(z)$ is well behaved at $\infty$ we require:

G2: The map:

$$
z \rightarrow G_{\cdot, j}^{a, \lambda}\left(z, z^{\prime}\right) \in L^{2}\left(\mathbf{R}^{2} \backslash B_{\rho}\right)
$$

for some $\rho>0$. Here $B_{\rho}$ denotes the ball of radius $\rho$ about 0 in $\mathbf{R}^{2}$.

Finally in order that the integral operator with kernel $G^{a, \lambda}\left(z, z^{\prime}\right)$ inverts $D_{a, \lambda}(m)$ away from the branch points we require:

G3: The difference of $G^{a, \lambda}\left(z, z^{\prime}\right)$ and the unperturbed Green function $G\left(z-z^{\prime}\right)$ should be smooth along the diagonal. That is:

$$
z \rightarrow G^{a, \lambda}\left(z, z^{\prime}\right)-G\left(z-z^{\prime}\right) \in C^{1}\left(N\left(z^{\prime}\right)\right)
$$

where $N\left(z^{\prime}\right)$ denotes a neighborhood of $z^{\prime}$ which is disjoint from the union of the branch cuts $b$.

REMARK. In SMJ III a "Green function" is introduced and characterized by just such conditions. The one difference in what we do here is in G1. Our restriction on the local expansions near the branch points $a_{j}$ is different than that in SMJ III.

It will take some work to establish the existence of a Green function but following SMJ we can easily show that the conditions $\mathbf{G} i$, for $i=1,2,3$ uniquely determine $G^{a, \lambda}$. The difference $\Delta\left(z, z^{\prime}\right)$ of two matrix valued solutions to $\mathbf{G} i$ for $i=1,2,3$, again satisfies $\mathrm{G} 1$ and $\mathrm{G} 2$ but $\mathrm{G} 3$ now implies that the columns of $z \rightarrow \Delta\left(z, z^{\prime}\right)$ extend to $z=z^{\prime}$ as solutions to the Dirac equation. Thus the columns of $\Delta\left(z, z^{\prime}\right)$ are multivalued solutions to the Dirac equation which are globally in $L^{2}\left(\mathbf{R}^{2}\right)$ and have local expansions of type (2.5) at the branch points. Proposition (1.4) implies that $\Delta\left(z, z^{\prime}\right)=0$.

Before we turn to the calculation of the "one-point" Green function we would like to show that the Green function $G^{a, \lambda}\left(z, z^{\prime}\right)$ can be characterized by its behavior in the $z^{\prime}$ variable. We will introduce a 
matrix $F^{a, \lambda}\left(z, z^{\prime}\right)$ which is characterized by the analogues of $\mathbf{G} i$ for $i=1,2,3$ for the second variable $z^{\prime}$. We will then do the standard calculation using Stokes' theorem that is used to prove the symmetry of Green functions in the classical case and we will find that:

$$
F^{a, \lambda}\left(z, z^{\prime}\right)=G^{a, \lambda}\left(z, z^{\prime}\right) \text {. }
$$

We define $F^{a, \lambda}$ by the following three conditions:

F1: The rows of the map $z^{\prime} \rightarrow F^{a, \lambda}\left(z, z^{\prime}\right)$ are multivalued solutions of the Dirac equation, defined for $z^{\prime} \in \mathbf{R}^{2} \backslash\{z\} \cup b$, and branched along $b_{j}$ with monodromy $\Lambda_{j}=e^{2 \pi i \lambda}$. These solutions have local expansions near $a_{\nu}$ given by:

$$
\begin{aligned}
& F_{j, .}^{a, \lambda}\left(z, z^{\prime}\right) \\
& \quad=\sum_{k>0}\left\{\alpha_{k, j}^{\nu}(z) w_{k+\lambda_{\nu}}\left(z^{\prime}-a_{\nu}\right)+\beta_{k, j}^{\nu}(z) w_{k-\lambda_{\nu}}^{*}\left(z^{\prime}-a_{\nu}\right)\right\}
\end{aligned}
$$

where the notation $F_{j}$, signifies the $j$ th row vector of the matrix $F$.

Note that the monodromy for $z^{\prime} \rightarrow F^{a, \lambda}\left(z, z^{\prime}\right)$ is the inverse of the monodromy for $z \rightarrow G^{a, \lambda}\left(z, z^{\prime}\right)$ at corresponding branch points.

F2: The map:

$$
z^{\prime} \rightarrow F_{j, .}^{a, \lambda}\left(z, z^{\prime}\right) \in L^{2}\left(\mathbf{R}^{2} \backslash B_{\rho}\right)
$$

for some $\rho>0$. Here $B_{\rho}$ denotes the ball of radius $\rho$ about 0 in $\mathbf{R}^{2}$.

F3: The difference of $F^{a, \lambda}\left(z, z^{\prime}\right)$ and the unperturbed Green function $G\left(z-z^{\prime}\right)$ should be smooth along the diagonal. That is:

$$
z^{\prime} \rightarrow F^{a, \lambda}\left(z, z^{\prime}\right)-G\left(z-z^{\prime}\right) \in C^{1}(N(z))
$$

where $N(z)$ denotes a neighborhood of $z$ which is disjoint from the union of the branch cuts $b$.

Proposition 2.0. The matrix valued functions $G^{a, \lambda}\left(z, z^{\prime}\right)$ and $F^{a, \lambda}\left(z, z^{\prime}\right)$ characterized by $\mathbf{G} i$ for $i=1,2,3$ and $\mathbf{F} i$ for $i=$ $1,2,3$ are equal. That is: $G^{a, \lambda}\left(z, z^{\prime}\right)=F^{a, \lambda}\left(z, z^{\prime}\right)$.

Proof. Suppose that $f$ and $g$ are smooth functions on some open set $U$ in $\mathbf{R}^{2}$, then it is easy to check that:

$$
\begin{gathered}
\{D(m) f(z) \cdot J g(z)-f(z) \cdot J D(m) g(z)\} i d z d \bar{z} \\
=d\left(f_{1}(z) g_{1}(z) d z+f_{2}(z) g_{2}(z) d \bar{z}\right) .
\end{gathered}
$$

Suppose that $x$ and $y$ are fixed elements of $\mathbf{R}^{2}$ which do not lie in $b$. Let $\varepsilon>0$ be chosen so that the ball of radius $\varepsilon$ about $x, B_{\varepsilon}(x)$, and 
the ball radius $\varepsilon$ about $y, B_{\varepsilon}(y)$, do not intersect the union of the branch cuts $b$. Let $N_{\varepsilon}\left(b_{j}\right)$ denote open neighborhoods of the branch cuts $b_{j}$ which are pairwise disjoint and do not intersect $B_{\varepsilon}(x) \cup B_{\varepsilon}(y)$. Finally let $N_{\varepsilon}$ denote the union of $B_{\varepsilon}(x) \cup B_{\varepsilon}(y)$ with $\bigcup_{j} N_{\varepsilon}\left(b_{j}\right)$. To show that $F^{a, \lambda}(x, y)$ and $G^{a, \lambda}(x, y)$ are the same we will set

$$
f(z)=F_{i, .}^{a, \lambda}(x, z) \text { and } g(z)=G_{\cdot, j}^{a, \lambda}(z, y)
$$

in (2.7) above. Since $D(m) f(z)=0$ and $D(m) g(z)=0$ for $z$ in the complement of $N_{\varepsilon}$ it follows that the left-hand side of (2.7) vanishes in this complement. Stokes' theorem shows that the integral of the left-hand side of (2.7) over the complement of $N_{\varepsilon}$ is given by the integral of a one form on the boundary. Thus:

$$
\int_{\partial N_{\varepsilon}} f_{1} g_{1} d z+f_{2} g_{2} d \bar{z}=0
$$

The boundary of $N_{\varepsilon}$ consists of the circles of radius $\varepsilon$ about $x$ and $y$ and the boundaries of the neighborhoods $N_{\varepsilon}\left(b_{j}\right)$. We haven't been specific about $N_{\varepsilon}\left(b_{j}\right)$ but now we observe that the exponential decay of $f(z)$ and $g(z)$ implied by $\mathrm{F} 2$ and $\mathrm{G} 2$ and Proposition (1.2) shows that we can collapse the boundary of each $N_{\varepsilon}\left(b_{j}\right)$ to a contour which comes in from $\infty$ along the top part of the ray $b_{j}$ makes a counter clockwise circuit of $a_{j}$ of radius $\varepsilon$ (for $\varepsilon$ sufficiently small) and goes back out to $\infty$ along the bottom of the ray $b_{j}$. Because $f(z)$ and $g(z)$ are branched solutions of the Dirac equation along each $b_{j}$ with monodromy for $f(z)$ that is precisely the inverse of the monodromy for $g(z)$ it follows that the contour integrals in (2.8) along the top and bottom portions of the ray $b_{j}$ exactly cancel-the contribution which each $\partial N_{\varepsilon}\left(b_{j}\right)$ makes to (2.8) is thus reduced to circuit integrals of radius $\varepsilon$ about the point $a_{j}$. A calculation of these circuit integrals using the local expansions for $f(z)$ and $g(z)$ that are described in $\mathrm{F} 1$ and $\mathrm{G} 1$ show that they all vanish in the limit $\varepsilon \rightarrow 0$. The only contributions to the circuit integral (2.8) which survive in the limit $\varepsilon \rightarrow 0$ are the contributions from $\partial B_{\varepsilon}(x)$ and $\partial B_{\varepsilon}(y)$. Now F3 and G3 imply that $F^{a, \lambda}(x, z)$ and $G^{a, \lambda}(z, y)$ differ from $G(x-z)$ and $G(z-y)$ by something which is continuous on the diagonal. The formula (2.1) for $G_{0}(z)$ in terms of Bessel functions and the asymptotics $K_{0}(z) \asymp-\log (z)$ and $K^{\prime}(z) \asymp-1 / z$ as $z \rightarrow 0$ now permit one to calculate the contributions to $(2.8)$ from $\partial B_{\varepsilon}(x)$ and $\partial B_{\varepsilon}(y)$ in the limit $\varepsilon \rightarrow 0$. One finds that the contribution from $\partial B_{\varepsilon}(x)$ is $G_{i, j}^{a, \lambda}(x, y)$ in this limit and the contribution from $\partial B_{\varepsilon}(y)$ is $-F_{i, j}^{a, \lambda}(x, y)$. This finishes the demonstration that $F^{a, \lambda}=G^{a, \lambda}$. 
$A$ Green function for $D_{a, \lambda}(m)$ with one branch point. We next consider the Dirac operator $D_{a, \lambda}(m)$ for a single branch point $a_{1}=a$ and monodromy parameter $\lambda_{1}=\lambda$. We will construct a Green function for $D_{a, \lambda}(m)$ which will serve as a left inverse for the restriction of $D_{a, \lambda}(m)$ to the functions in its domain which are mapped to zero in a neighborhood of the branch cut emerging from $a$.

That $D_{a, \lambda}(m)$ is invertible is not too surprising. The operator $D_{a, \lambda}$ is formally skew symmetric and to say that $D_{a, \lambda}(m)$ is invertible is roughly the same as saying that the real number $-m / 2 \neq 0$ is in the resolvent set for $D_{a, \lambda}$. This same "argument" applies in the case that there are $n$ branch points to show that $D_{a, \lambda}(m)$ is invertible. I have not yet found a way to make this rigorous (what is missing is a characterization of the range). Our construction of a Green function for $D_{a, \lambda}(m)$ will stop short of the estimates needed to prove that $D_{a, \lambda}(m)$ is indeed invertible. The existence of the Green function will suffice to discuss the localizations of $D_{a, \lambda}(m)$ which are the main objects of interest for us, and is simpler to deal with.

We return now to the consideration of the case in which there is a single branch point $a$. For simplicity we may take the branch point to be at $a=0$ since we may obtain the general result from this special case by translation. Let $D(m)$ denote the Dirac operator:

$$
D(m):=D+\frac{m}{2} I \text {. }
$$

We will begin with a heuristic account of the "derivation" of a Green function for $D_{a, \lambda}(m)$ and then we will confirm that the formula we obtain actually works.

Suppose that $f$ is in the domain of $D_{0, \lambda}(m)$. To avoid any subtlety in the following calculations we suppose that $f$ is once differentiable away from the branch cut on the positive real axis and that it has continuous boundary values on the branch cut (though not necessarily at the vertex 0$)$. In the case of principal interest to us $D_{0, \lambda}(m) f(x)$ will vanish in a neighborhood of the branch cut and $f$ will have continuous boundary values there.

We can think of the Green function $G^{0, \lambda}\left(z, z^{\prime}\right)$ as a pair of vector valued functions $G_{i, .}^{0, \lambda}\left(z, z^{\prime}\right)$ for $i=1,2$ on $\mathbf{R}^{2} \backslash \mathbf{R}^{+} \times \mathbf{R}^{2} \backslash \mathbf{R}^{+}$such that (see 2.3):

$$
\int_{\mathbf{R}^{2} \backslash \mathbf{R}^{+}} G_{i, \cdot}^{0, \lambda}\left(z, z^{\prime}\right) \cdot J D_{0, \lambda}(m) f\left(z^{\prime}\right) i d z^{\prime} d \bar{z}^{\prime}=f_{i}(z)
$$

where $\mathbf{R}^{+}$is the positive real axis and it is excluded to remind the reader that the function $f$ has a branch cut along $\mathbf{R}^{+}$. Since $f$ is a 
smooth function away from this branch cut we have $D_{0, \lambda}(m) f(y)=$ $D(m) f(y)$ for $y \notin \mathbf{R}^{+}$. Now let $r=|z|$ and define $I_{\varepsilon}^{-}=B_{r-\varepsilon} \backslash \mathbf{R}^{+}$ to be the ball of radius $r-\varepsilon$ about 0 with that portion of the positive real axis that lies inside this ball deleted. Let $E_{\varepsilon}^{+}=\mathbf{R}^{2} \backslash\left\{B_{r+\varepsilon} \cup \mathbf{R}^{+}\right\}$ denote the exterior of the ball of radius $r+\varepsilon$ with that portion of the positive real axis that lies inside this exterior region deleted. Stokes' theorem and equation (1.12) then imply that the integrals:

$$
\int_{I_{\varepsilon}^{-}} G_{i, \cdot}^{0, \lambda}\left(z, z^{\prime}\right) \cdot J D(m) f\left(z^{\prime}\right) i d z^{\prime} d \bar{z}^{\prime}
$$

and

$$
\int_{E_{\varepsilon}^{+}} G_{i, \cdot}^{0, \lambda}\left(z, z^{\prime}\right) \cdot J D(m) f\left(z^{\prime}\right) i d z^{\prime} d \bar{z}^{\prime}
$$

reduce to the boundary integrals:

$$
\int_{\partial I_{\varepsilon}^{-}} G_{i 1}^{0, \lambda}\left(z, z^{\prime}\right) f_{1}\left(z^{\prime}\right) d z^{\prime}+G_{i 2}^{0, \lambda}\left(z, z^{\prime}\right) f_{2}\left(z^{\prime}\right) d \bar{z}^{\prime}
$$

and

$$
\int_{\partial E_{\varepsilon}^{+}} G_{i 1}^{0, \lambda}\left(z, z^{\prime}\right) f_{1}\left(z^{\prime}\right) d z^{\prime}+G_{i 2}^{0, \lambda}\left(z, z^{\prime}\right) f_{2}\left(z^{\prime}\right) d \bar{z}^{\prime}
$$

since $z^{\prime} \rightarrow G_{i, .}^{0, \lambda}\left(z, z^{\prime}\right)$ is a non singular solution to the Dirac equation inside the sets $I_{\varepsilon}^{-}$and $E_{\varepsilon}^{-}$. In the limit $\varepsilon \rightarrow 0$ the sum of these two contour integrals should reproduce $f_{i}(z)$. The boundary $\partial I_{\bar{\varepsilon}}^{-}$and the boundary $\partial E_{\varepsilon}^{+}$each consist of two pieces-one piece a circle of radius $r \pm \varepsilon$ and the other piece made up of the "top" and "bottom" portions of the positive real axis that are included in $I_{\varepsilon}^{-}$and $E_{\varepsilon}^{+}$. The integrals over the top and bottom portions of the real axis will cancel provided $f_{j}\left(z^{\prime}\right) G_{i j}^{0, \lambda}\left(z, z^{\prime}\right)$ is continuous across the real axis. This will happen for smooth $f$ if:

$$
u \rightarrow\left(\begin{array}{cc}
u^{-\lambda} & 0 \\
0 & \bar{u}^{\lambda}
\end{array}\right) G_{i, \cdot}^{0, \lambda}(z, u)
$$

is continuous across the positive real axis and this is, in turn, a consequence of the local expansion F1 for the Green function.

Finally, the sum of the two remaining circuit integrals should reproduce the function $f_{i}(z)$ in the limit $\varepsilon \rightarrow 0$. Let $(r, \theta)$ denote the standard polar coordinates of a point in $\mathbf{R}^{2}$. Then we will achieve the result we desire if:

$$
\left\{G_{i 1}^{0, \lambda}\left(r, \theta, r+, \theta^{\prime}\right)-G_{i 1}^{0, \lambda}\left(r, \theta, r-, \theta^{\prime}\right)\right\} i e^{i \theta^{\prime}} r=\delta_{i 1} \delta\left(\theta-\theta^{\prime}\right)
$$


and

$$
\left\{G_{i 2}^{0, \lambda}\left(r, \theta, r+, \theta^{\prime}\right)-G_{i 2}^{0, \lambda}\left(r, \theta, r-, \theta^{\prime}\right)\right\} i e^{-i \theta^{\prime}} r=\delta_{l 2} \delta\left(\theta-\theta^{\prime}\right)
$$

where $r+$ is the limit from above $r$ and $r$ - is the limit from below $r$. This translation of the condition $\mathrm{F} 3$ for $G^{0, \lambda}\left(z, z^{\prime}\right)$ into the jump conditions (2.11) and (2.12) for $G^{0, \lambda}\left(z, z^{\prime}\right)$ is the main point of this exercise. Now we will use this to find an explicit formula for $G^{0, \lambda}\left(z, z^{\prime}\right)$. We begin by writing down the local expansion for $G_{i, .}^{0, \lambda}\left(z, z^{\prime}\right)$ in $z^{\prime}$ near 0 :

$$
G_{i, .}^{0, \lambda}\left(z, z^{\prime}\right)=\sum_{k>0}\left\{\alpha_{k, i}(z, \lambda) w_{k+\lambda}\left(z^{\prime}\right)+\beta_{k, i}(z, \lambda) w_{k-\lambda}^{*}\left(z^{\prime}\right)\right\} .
$$

The components of the Green function $G_{i, .}^{0, \lambda}\left(z, z^{\prime}\right)$ that we are interested in should tend to 0 as $z^{\prime}$ tends to $\infty$ in the plane. Recalling Proposition (1.2) we have:

$$
G_{i, .}^{0, \lambda}\left(z, z^{\prime}\right)=\sum_{k \in Z+1 / 2} \gamma_{k, i}(z, \lambda) \hat{w}_{k-\lambda}\left(z^{\prime}\right) .
$$

Our goal is to calculate the coefficients $\alpha_{k, l}(z, \lambda), \beta_{k, i}(z, \lambda)$, and $\gamma_{k, i}(z, \lambda)$. Since the columns of the Green function should be multivalued solutions to the Dirac equation as well as the rows it is fairly clear that the vector valued functions:

$$
\begin{gathered}
\alpha_{k}(z, \lambda):=\left(\begin{array}{c}
\alpha_{k, 1}(z, \lambda) \\
\alpha_{k, 2}(z, \lambda)
\end{array}\right), \quad \beta_{k}(z, \lambda):=\left(\begin{array}{c}
\beta_{k, 1}(z, \lambda) \\
\beta_{k, 2}(z, \lambda)
\end{array}\right), \\
\gamma_{k}(z, \lambda):=\left(\begin{array}{l}
\gamma_{k, 1}(z, \lambda) \\
\gamma_{k, 2}(z, \lambda)
\end{array}\right)
\end{gathered}
$$

ought to be multivalued solutions of the Dirac equation branched along $\mathbf{R}^{+}$with monodromy $e^{-2 \pi i \lambda}$. To further specify these functions observe that $D_{0, \lambda}(m)$ acts like $D(m)$ away from the branch cut on the positive real axis and so commutes with the infinitesimal rotation $R$ (apart from domain considerations). Thus the operator associated with $G^{0, \lambda}$ ought to "commute" with $R$. One may check that $R$ is formally skew symmetric with respect to the pairing associated with the bilinear form $(\cdot, \cdot)$ and the measure $i d z d \bar{z}$. Using this to express the fact that $G^{0, \lambda}$ and $R$ commute one finds:

$$
R \alpha_{k}(z, \lambda)=-(k+\lambda) \alpha_{k}(z, \lambda) \quad R \beta_{k}(z, \lambda)=(k-\lambda) \beta_{k}(z, \lambda)
$$

and

$$
R \gamma_{k}(z, \lambda)=(k-\lambda) \gamma_{k}(z, \lambda)
$$


Thus the multivalued solutions to the Dirac equation that we seek are all eigenfunctions for the infinitesimal rotation $R$. Clearly $\mathrm{G} 2$ suggests the functions $\alpha_{k}(z, \lambda)$ and $\beta_{k}(z, \lambda)$ ought to be square integrable at $\infty$ and so we have:

$$
\alpha_{k}(z, \lambda)=\alpha_{k, \lambda} \hat{w}_{k+\lambda}(z) \text { and } \beta_{k}(z, \lambda)=\beta_{k, \lambda} \hat{w}_{-k+\lambda}(z)
$$

for some constants $\alpha_{k, \lambda}$ and $\beta_{k, \lambda}$. The function $\gamma_{k}(z, \lambda)$ need not be square integrable at infinity but it must not be too singular at $z=0$. Thus G1 suggests:

$$
\gamma_{k}(z, \lambda)=a_{k, \lambda} w_{k-\lambda}(z)+b_{k, \lambda} w_{k+\lambda}^{*}(z)
$$

for some constants $a_{k, \lambda}$ and $b_{k, \lambda}$ which are zero for $k<0$. We now assemble these observations into formulas for the Green function:

$$
\begin{aligned}
& G^{0, \lambda}\left(z, z^{\prime}\right) \\
& \quad=\sum_{k>0}\left\{\alpha_{k, \lambda} \hat{w}_{k+\lambda}(z) \otimes w_{k+\lambda}\left(z^{\prime}\right)+\beta_{k, \lambda} \hat{w}_{-k+\lambda}(z) \otimes w_{k-\lambda}^{*}\left(z^{\prime}\right)\right\}
\end{aligned}
$$

for all $\left|z^{\prime}\right|<|z|$ and

$$
\begin{aligned}
& G^{0, \lambda}\left(z, z^{\prime}\right) \\
& \quad=\sum_{k>0}\left\{a_{k, \lambda} w_{k-\lambda}(z) \otimes \hat{w}_{k-\lambda}\left(z^{\prime}\right)+b_{k, \lambda} w_{k+\lambda}^{*}(z) \otimes \hat{w}_{-k-\lambda}\left(z^{\prime}\right)\right\}
\end{aligned}
$$

for all $\left|z^{\prime}\right|>|z|$. In each of these formulas the tensor product notation is used to signify the usual matrix product of a column vector with a row vector:

$$
A \otimes B=\left[\begin{array}{ll}
A_{1} B_{1} & A_{1} B_{2} \\
A_{2} B_{1} & A_{2} B_{2}
\end{array}\right]
$$

In order to determine the constants $\alpha_{k, \lambda}, \beta_{k, \lambda}, a_{k, \lambda}$ and $b_{k, \lambda}$ in these formulas we now enforce (2.11) and (2.12) making use of the Wronskian for modified Bessel functions:

$$
I_{k}(r) I_{1-k}(r)-I_{-k}(r) I_{k-1}(r)=-\frac{2 \sin (k \pi)}{\pi r} .
$$

One finds:

$$
a_{k, \lambda}=-\beta_{k, \lambda} \text { and } b_{k, \lambda}=-\alpha_{k, \lambda}
$$

with:

$$
\alpha_{k, \lambda}=-\beta_{k, \lambda}=\frac{-i m(-1)^{k+1 / 2}}{4 \sin (\pi \lambda)}
$$


The expansions that result for the Green function are:

$$
\begin{aligned}
G^{0, \lambda}\left(z, z^{\prime}\right)= & \frac{i m}{4 \sin (\pi \lambda)} \sum_{k>0}(-1)^{k-1 / 2} \\
& \cdot\left\{\hat{w}_{k+\lambda}(z) \otimes w_{k+\lambda}\left(z^{\prime}\right)-\hat{w}_{-k+\lambda}(z) \otimes w_{k-\lambda}^{*}\left(z^{\prime}\right)\right\}
\end{aligned}
$$

for $\left|z^{\prime}\right|<|z|$ and

$$
\begin{aligned}
G^{0, \lambda}\left(z, z^{\prime}\right)= & \frac{i m}{4 \sin (\pi \lambda)} \sum_{k>0}(-1)^{k-1 / 2} \\
& \cdot\left\{w_{k-\lambda}(z) \otimes \hat{w}_{k-\lambda}\left(z^{\prime}\right)-w_{k+\lambda}^{*}(z) \otimes \hat{w}_{-k-\lambda}\left(z^{\prime}\right)\right\}
\end{aligned}
$$

for $\left|z^{\prime}\right|>|z|$. We will check that these formulas make sense (i.e., converge in the appropriate sense) and represent the Green function in just a moment. At this point we can do a calculation in the one point case (at least formally) that is the fundamental result in our version of the SMJ analysis. The Green function for $D_{a, \lambda}$ is $G^{a, \lambda}\left(z, z^{\prime}\right)=G^{0, \lambda}\left(z-a, z^{\prime}-a\right)$ obtained from the Green function above by translation. Using either (2.13) or (2.14) one finds the derivative of this Green function with respect to the parameter $a$ is:

$$
\begin{aligned}
\partial_{a} G^{a, \lambda}\left(z, z^{\prime}\right) & =\frac{i m^{2}}{8 \sin (\pi \lambda)} \hat{w}_{1 / 2+\lambda}(z-a) \otimes \hat{w}_{1 / 2-\lambda}\left(z^{\prime}-a\right) \\
\bar{\partial}_{a} G^{a, \lambda}\left(z, z^{\prime}\right) & =\frac{i m^{2}}{8 \sin (\pi \lambda)} \hat{w}_{-1 / 2+\lambda}(z-a) \otimes \hat{w}_{-1 / 2-\lambda}\left(z^{\prime}-a\right) .
\end{aligned}
$$

The analogous result for the Green function with $n$-branch points will be a principal object in the third section of this paper.

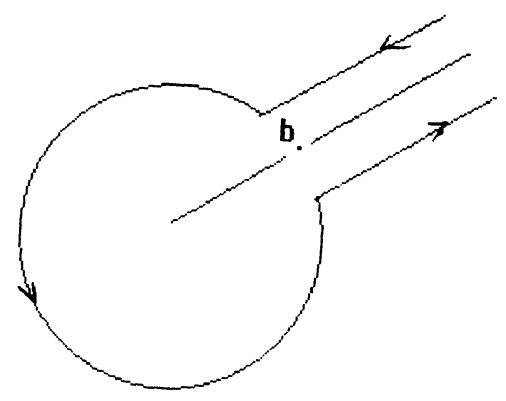

FIGURE 1 


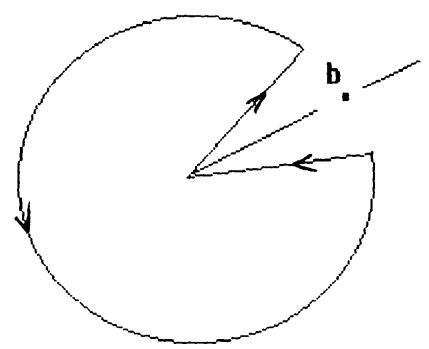

FIGURE 2

In order to discuss the convergence of the infinite series (2.13) and (2.14) for the Green function and to prove that they do give a representation for the kernel of the inverse of the appropriate operator it will be very convenient to introduce contour integral representations for the wave functions $w_{l}(z)$ and $w_{l}^{*}(z)$. We first discuss the two sorts of contours that will be important for us. Let $b$ denote a non zero point in $\mathbf{C}$ and write $b \mathbf{R}^{+}=\{\alpha b \mid \alpha>0\}$ for the ray through 0 and $b$. We will write $C_{1}^{\varepsilon}(b)$ for the contour that surrounds $b \mathbf{R}^{+}$ in a counter-clockwise manner in the following fashion. The contour $C_{1}^{\varepsilon}(b)$ follows the ray parallel to $b \mathbf{R}^{+}$from $\infty$ to $e^{i \varepsilon} b$; it then follows the circle of radius $|b|$ from $e^{i \varepsilon} b$ counter-clockwise to $e^{-i \varepsilon} b$; finally it travels from $e^{-i \varepsilon} b$ along the ray parallel to $b \mathbf{R}^{+}$out to $\infty$ (see Figure 1). The contour $C_{2}^{\varepsilon}(b)$ starts at 0 and follows the line to $e^{i \varepsilon} b$; it then makes a counter-clockwise circuit from $e^{i \varepsilon} b$ to $e^{-i \varepsilon} b$ along the circle of radius $|b|$; finally it returns to the origin along the line which joins $e^{-i \varepsilon} b$ to 0 (see Figure 2). Now suppose that $f(v)$ is a holomorphic function for $v \in \mathbf{C} \backslash b \mathbf{R}^{+}$and define:

$$
\int_{C_{k}(b)} f(v) d v=\lim _{\varepsilon \rightarrow 0} \int_{C_{k}^{\varepsilon}(b)} f(v) d v
$$

provided, of course, that the limit makes sense. We are exclusively interested in the case where the function $f(v)$ has boundary values on the ray $b \mathbf{R}^{+}$which depend, however, on the side the ray is approached from. To describe these functions we introduce a logarithm, $\log _{b}$, with a branch cut on the ray $b \mathbf{R}^{+}$by:

$$
\arg (b)-2 \pi<\operatorname{Im}\left(\log _{b}(z)\right)<\arg (b)
$$

for $z$ not in $b \mathbf{R}^{+}$and $\arg (b)$ chosen so that:

$$
0 \leq \arg (b)<2 \pi \text {. }
$$

Now define:

$$
e(z, v)=e^{-(m / 2)\left(\bar{z} v+z v^{-1}\right)}
$$


and let $\operatorname{HP}(b)$ denote the half plane in $\mathbf{C}$ consisting of those vectors, $z$, with positive Euclidean inner product $z \cdot b=z_{1} b_{1}+z_{2} b_{2}>0$ with $b$. If $b$ is chosen in the left half plane and $z \in \operatorname{HP}(b)$ then:

$$
w_{l}(z)=e^{i \pi(l-1 / 2)} \int_{C_{2}(b)} d v \frac{e(z, v) v^{l-1 / 2}}{2 \pi i v}\left(\begin{array}{c}
1 \\
-v
\end{array}\right)
$$

and

$$
w_{l}^{*}(z)=e^{-i \pi(l+1 / 2)} \int_{C_{1}(b)} d v \frac{e(z, v) v^{-l-1 / 2}}{2 \pi i v}\left(\begin{array}{c}
1 \\
-v
\end{array}\right) .
$$

In each integral the fractional powers of $v$ are defined by:

$$
v^{\alpha}:=e^{\alpha \log _{b}(v)} .
$$

The restriction $z \cdot b>0$ implies both that $\operatorname{Re}(\bar{z} v)>0$ and $\operatorname{Re}\left(z v^{-1}\right)$ $>0$ for all $v$ that lie on $b \mathbf{R}^{+}$. This implies that the exponential factor $e(z, v)$ controls the polynomial singularities in the integrands at both 0 (for $C_{2}(b)$ ) and $\infty$ (for $C_{1}(b)$ ). We restrict $b$ to the left half plane to single out a branch of the multivalued functions $w_{l}(z)$ and $w_{l}^{*}(z)$. Only for points $z$ on the positive real axis or for $z=0$ is it not possible to find a $b$ in the left half plane with $z \cdot b>0$. We leave it to the reader to check that this choice of a branch for $w_{l}$ and $w_{l}^{*}$ corresponds to restricting $\theta$ in (1.0) and (1.1) to values between 0 and $2 \pi$.

Cauchy's theorem implies that one may choose any $b \in \operatorname{HP}(z)$ in the contour representations for $w_{l}(z)$ and $w_{l}^{*}(z)$ without changing the values of the integrals. One obvious choice that will be useful for us is to choose $b=\alpha z$ for some $\alpha>0$. Thus:

$$
w_{l}(z)=e^{i \pi(l-1 / 2)} \int_{C_{2}(\alpha z)} d v \frac{e(z, v) v^{l-1 / 2}}{2 \pi i v}\left(\begin{array}{c}
1 \\
-v
\end{array}\right)
$$

and

$$
w_{l}^{*}(z)=e^{-i \pi(l+1 / 2)} \int_{C_{1}(\alpha z)} d v \frac{e(z, v) v^{-l-1 / 2}}{2 \pi i v}\left(\begin{array}{c}
1 \\
-v
\end{array}\right)
$$

where in each of the two preceding representations $z \in \mathbf{C} \backslash \mathbf{R}^{+}$. These representations of $w_{l}(z)$ and $w_{l}^{*}(z)$ allow us to obtain a useful reprèsentation of $\hat{w}_{l}(z)=w_{l}^{*}(z)-w_{-l}(z)$ as well. Let $C(b)=C_{2}(b)-C_{1}(b)$ (strictly speaking, we ought to define $C(b)$ as a limit just as $C_{k}(b)$ was defined). The circular arcs in $C_{1}(b)$ and $C_{2}(b)$ cancel and we find that $C(b)$ consists of the difference of the two sides of the ray $b \mathbf{R}^{+}$. 
The representation for $\hat{w}_{l}(z)$ that arises by subtracting the preceding representations for $w_{l}^{*}(z)$ and $w_{-l}(z)$ is then:

$$
\hat{w}_{l}(z)=e^{-i \pi(l+1 / 2)} \int_{C(\alpha z)} d v \frac{e(z, v) v^{-l-1 / 2}}{2 \pi i v}\left(\begin{array}{c}
1 \\
-v
\end{array}\right) .
$$

We may also write this last integral as:

$$
\hat{w}_{l}(z)=2 i \sin \left(\pi\left(l+\frac{1}{2}\right)\right) \int_{z \mathbf{R}^{+}} d v \frac{e(z, v) v^{-l-1 / 2}}{2 \pi i v}\left(\begin{array}{c}
1 \\
-v
\end{array}\right)
$$

where it is understood in the last integral that the fractional power of $v$ which occurs is determined by the principal value $0 \leq \arg (v)<$ $2 \pi$. We are now prepared to use these representations to establish the convergence of the infinite series for the Green function given above. For simplicity we will treat the case $\left|z^{\prime}\right|<|z|$ in detail and only indicate the changes needed to handle the other case. Substituting (2.19) into (2.13) one finds the two sums:

$$
\frac{m}{2} \sum_{k>0} \int_{z \mathbf{R}^{+}} d u \frac{e(z, u) u^{-\lambda}}{2 \pi i u}\left(\begin{array}{c}
1 \\
-u
\end{array}\right) \otimes u^{-k-1 / 2} w_{k+\lambda}\left(z^{\prime}\right)
$$

and

$$
-\frac{m}{2} \sum_{k>0} \int_{z \mathbf{R}^{+}} d u \frac{e(z, u) u^{-\lambda}}{2 \pi i u}\left(\begin{array}{c}
1 \\
-u
\end{array}\right) \otimes u^{k-1 / 2} w_{k-\lambda}^{*}\left(z^{\prime}\right)
$$

where once again the fractional powers of $u$ in (2.20) and (2.21) are determined by the principal value $0 \leq \arg (u)<2 \pi$ of the argument. If we truncate the $k$ sum in (2.20) and interchange the finite sum and the integral we encounter the sum:

$$
F_{N}(u):=\sum_{0<k<N} u^{-k-1 / 2} w_{k+\lambda}\left(z^{\prime}\right)
$$

where $N$ is a positive integer. Substituting the representation (2.15) for $w_{k+\lambda}\left(z^{\prime}\right)$ into this last sum one finds:

$$
F_{N}(u)=e^{i \pi \lambda} \int_{C_{2}\left(\alpha z^{\prime}\right)} d v \frac{e\left(z^{\prime}, v\right) v^{\lambda}}{2 \pi i v} \frac{1-(-v / u)^{N}}{u+v}\left(\begin{array}{c}
1 \\
-v
\end{array}\right) .
$$

If we now pick the constant $\alpha$ so that $\left|\alpha z^{\prime}\right|<|u|$ then $|v / u|<1$ for all $v \in C_{2}\left(\alpha z^{\prime}\right)$ and it is clear that:

$$
\lim _{N \rightarrow \infty} F_{N}(u)=e^{i \pi \lambda} \int_{C_{2}\left(\alpha z^{\prime}\right)} d v \frac{e\left(z^{\prime}, v\right) v^{\lambda}}{2 \pi i v}(u+v)^{-1}\left(\begin{array}{c}
1 \\
-v
\end{array}\right) .
$$


With a slightly more careful choice of $\alpha$ we can estimate the convergence of $F_{N}(u)$ to its limit so that dominated convergence applies in the $u$ integral. For this we will use the fact that $\left|z^{\prime}\right|<|z|$. Fix $\varepsilon>0$ so that:

$$
\left|z^{\prime}\right|<(1-\varepsilon)^{2}|z|
$$

and then choose:

$$
\alpha=(1-\varepsilon)\left|u / z^{\prime}\right|
$$

The very simplest estimates now show that the contribution to $F_{N}(u)$ which arises from the integration over the circle in $C_{2}\left(\alpha z^{\prime}\right)$ is dominated by a constant multiple of:

$$
\varepsilon^{-1}|u|^{\lambda-n} e^{(m / 2)(1-\varepsilon)\left(\bar{z} u+z u^{-1}\right)}
$$

where $n=2$ or $n=1$ depending on whether one looks at the first or the second component of $F_{N}(u)$. The contribution to $F_{N}(u)$ which arises from the line segments in $C_{2}\left(\alpha z^{\prime}\right)$ is even easier to bound since the exponential term is always favorable and one finds it is dominated by a constant times $\varepsilon^{-1}$. These two estimates suffice to show that $F_{N}(u)$ is controlled by the decaying exponential factor $e^{-(m / 2)\left(\bar{z} u+z u^{-1}\right)}$ in the integrand; dominated convergence applies to interchange the $k$ sum with the $u$ integral in (2.20) and one obtains for (2.20) the iterated integral:

$$
\begin{aligned}
& \frac{m e^{i \pi \lambda}}{2} \int_{z \mathbf{R}^{+}} d u \frac{e(z, u) u^{-\lambda}}{2 \pi i u} \\
& \cdot \int_{C_{2}\left(\alpha z^{\prime}\right)} d v \frac{e\left(z^{\prime}, v\right) v^{\lambda}}{2 \pi i v}(u+v)^{-1}\left[\begin{array}{cc}
1 & -u \\
-v & u v
\end{array}\right]
\end{aligned}
$$

where in this last formula $\alpha$ need only be chosen so that $\alpha\left|z^{\prime}\right|<|u|$.

A similar formula results for the sum in (2.21) with the difference that the contour over which the interior integral must be done is $C_{1}\left(\beta z^{\prime}\right)$ with $\beta\left|z^{\prime}\right|>|u|$ and the overall sign is different. Combining these two results one finds that for $\left|z^{\prime}\right|<|z|$ the Green function $G^{0, \lambda}\left(z, z^{\prime}\right)$ is given by:

$$
\begin{aligned}
& \frac{m e^{i \pi \lambda}}{2} \int_{z \mathbf{R}^{+}} d u \frac{e(z, u) u^{-\lambda}}{2 \pi i u} \\
& \quad \cdot \int_{C_{\alpha \beta}} d v \frac{e\left(z^{\prime}, v\right) v^{\lambda}}{2 \pi i v}(u+v)^{-1}\left[\begin{array}{cc}
1 & -u \\
-v & u v
\end{array}\right]
\end{aligned}
$$

where the contour $C_{\alpha \beta}=C_{2}\left(\alpha z^{\prime}\right)-C_{1}\left(\beta z^{\prime}\right)$ subject to the condition:

$$
\alpha\left|z^{\prime}\right|<|u|<\beta\left|z^{\prime}\right|
$$

on $\alpha$ and $\beta$. 
This integral representation may be simplified somewhat by deforming the contour $C_{1}\left(\beta z^{\prime}\right)$ to $C_{1}\left(\alpha z^{\prime}\right)$ in the interior integral. One finds a contribution from the pole at $v=-u$ and the contour $C_{2}\left(\alpha z^{\prime}\right)-$ $C_{1}\left(\beta z^{\prime}\right)$ becomes $C_{2}\left(\alpha z^{\prime}\right)-C_{1}\left(\alpha z^{\prime}\right)=C\left(\alpha z^{\prime}\right)=C\left(z^{\prime}\right)$. In the evaluation of the residue term one must find the value of $v^{\lambda} / u^{\lambda}$ at $v=-u$ for $u$ on the contour $z \mathbf{R}^{+}$. We will now describe this.

The appropriate choice of a branch for $v^{\lambda}$ on the curve $C_{1}\left(\beta z^{\prime}\right)$ is determined by the branch of the logarithm $\log _{z^{\prime}}(v)$. Thus:

$$
\left.v^{\lambda}\right|_{v=-u}=|u|^{\lambda} e^{i \lambda \operatorname{Im}\left(\log _{z^{\prime}}(-u)\right)} \text {. }
$$

But $\operatorname{Im}\left(\log _{z^{\prime}}(-u)\right)=\operatorname{Im}\left(\log _{z^{\prime}}(-z)\right)$ and by definition:

$$
\arg \left(z^{\prime}\right)-2 \pi<\operatorname{Im}\left(\log _{z^{\prime}}(-z)\right) \leq \arg \left(z^{\prime}\right)
$$

where $\arg (z)$ denotes the branch of the argument function in the complex plane chosen so that $0 \leq \arg (z)<2 \pi$. It is clear that for all $z, z^{\prime} \in \mathbf{C}$ which are not on the positive real axis or zero we have:

$$
-2 \pi<\arg \left(z^{\prime}\right)-\arg (z)<2 \pi .
$$

The reader may now easily check that the following choices are appropriate for $\operatorname{Im}\left(\log _{z^{\prime}}(-z)\right)$.

$$
\operatorname{Im}\left(\log _{z^{\prime}}(-z)\right)= \begin{cases}\arg (z)-3 \pi & \text { for } \arg \left(z^{\prime}\right)-\arg (z) \in(-2 \pi,-\pi), \\ \arg (z)-\pi & \text { for } \arg \left(z^{\prime}\right)-\arg (z) \in(-\pi, \pi), \\ \arg (z)+\pi & \text { for } \arg \left(z^{\prime}\right)-\arg (z) \in(\pi, 2 \pi) .\end{cases}
$$

One finds:

$$
v^{\lambda} /\left.u^{\lambda}\right|_{v=-u}= \begin{cases}e^{-3 i \pi \lambda} & \text { for } \arg \left(z^{\prime}\right)-\arg (z) \in(-2 \pi,-\pi), \\ e^{-i \pi \lambda} & \text { for } \arg \left(z^{\prime}\right)-\arg (z) \in(-\pi, \pi), \\ e^{i \pi \lambda} & \text { for } \arg \left(z^{\prime}\right)-\arg (z) \in(\pi, 2 \pi) .\end{cases}
$$

These results can be used to calculate the residue that arises in deforming the contour $C_{1}\left(\beta z^{\prime}\right)$ to $C_{1}\left(\alpha z^{\prime}\right)$ in the inner integral of $(2.23)$. The case of principal interest to us is the case in which the arguments of $z$ and $z^{\prime}$ differ by less than $\pi$ in absolute value (this is always the case, for example, when $z$ and $z^{\prime}$ are sufficiently close to one another). In this case the residue contribution is:

$$
\frac{m}{2} \int_{z \mathbf{R}^{+}} d u \frac{e\left(z-z^{\prime}, u\right)}{2 \pi i u}\left[\begin{array}{cc}
-u^{-1} & -1 \\
1 & u
\end{array}\right] \text {. }
$$

Note that when $\left|z^{\prime}\right|<|z|$ it is true that $z \cdot\left(z-z^{\prime}\right)>0$ so that the exponential factor in this last integrand is decaying at both 0 and 
$\infty$. Thus the ray $z \mathbf{R}^{+}$can be deformed to the ray $\left(z-z^{\prime}\right) \mathbf{R}^{+}$in this last integral and we find the representation (2.0) for the free Green function in its modified form (2.2).

Thus for $\left|z^{\prime}\right|<|z|$ and $-\pi<\arg \left(z^{\prime}\right)-\arg (z)<\pi$ we have:

$$
G^{0, \lambda}\left(z, z^{\prime}\right)=G\left(z-z^{\prime}\right)+\Delta\left(z, z^{\prime}\right)
$$

where:

$$
\begin{aligned}
\Delta\left(z, z^{\prime}\right)= & -i m \sin (\pi \lambda) \int_{z \mathbf{R}^{+}} d u \frac{e(z, u) u^{-\lambda}}{2 \pi i u} \\
& \cdot \int_{z^{\prime} \mathbf{R}^{+}} d v \frac{e\left(z^{\prime}, v\right) v^{\lambda}}{2 \pi i v}(u+v)^{-1}\left[\begin{array}{cc}
1 & -v \\
-u & u v
\end{array}\right]
\end{aligned}
$$

and the fractional powers $u^{-\lambda}$ and $v^{\lambda}$ are determined by the principal value arguments $\arg (u)$ and $\arg (v)$ each between 0 and $2 \pi$. The only change in (2.24) needed to accommodate the cases where $\arg \left(z^{\prime}\right)-\arg (z)$ is in the intervals $(-2 \pi,-\pi)$ or $(\pi, 2 \pi)$ is that $G\left(z-z^{\prime}\right)$ on the right-hand side of (2.24) should be replaced by $e^{-2 \pi i \lambda} G\left(z-z^{\prime}\right)$ and $e^{2 \pi i \lambda} G\left(z-z^{\prime}\right)$ respectively. Thus

$$
G^{0, \lambda}\left(z, z^{\prime}\right)=\left\{\begin{array}{c}
e^{-2 \pi i \lambda} G\left(z-z^{\prime}\right)+\Delta\left(z, z^{\prime}\right) \\
\quad \text { or } \arg \left(z^{\prime}\right)-\arg (z) \in(-2 \pi,-\pi), \\
G\left(z-z^{\prime}\right)+\Delta\left(z, z^{\prime}\right) \\
\quad \text { for } \arg \left(z^{\prime}\right)-\arg (z) \in(-\pi, \pi), \\
e^{2 \pi i \lambda} G\left(z-z^{\prime}\right)+\Delta\left(z, z^{\prime}\right) \\
\quad \text { for } \arg \left(z^{\prime}\right)-\arg (z) \in(\pi, 2 \pi) .
\end{array}\right.
$$

We are now prepared to check that $G^{0, \lambda}\left(z, z^{\prime}\right)$ is a Green function for $D_{0, \lambda}(m)$ in the sense of conditions $\mathbf{G} i, i=1,2,3$. Perhaps the simplest condition to check is G3. Formula (2.25) shows that $G^{0, \lambda}\left(z, z^{\prime}\right)$ differs from $G\left(z-z^{\prime}\right)$ by $\Delta\left(z, z^{\prime}\right)$ for $z$ sufficiently near $z^{\prime}$. When $z$ is near $z^{\prime}$ it is a simple matter to fix both the contours in (2.24) to $z^{\prime} \mathbf{R}^{+}$. One sees then that $\Delta\left(z, z^{\prime}\right)$ is a smooth function of $z$ for all $z$ sufficiently near $z^{\prime}$. Our proof of the convergence of $(2.13)$ for $|z|>\left|z^{\prime}\right|$ shows that the columns of $G^{0, \lambda}\left(z, z^{\prime}\right)$ have a convergent expansion of type (1.11) for $|z|$ sufficiently large. Thus Proposition 1.2 implies that the columns of $G^{0, \lambda}\left(z, z^{\prime}\right)$ are square integrable (in $\left.z\right)$ in a neighborhood of $\infty$. This confirms that $G^{0, \lambda}\left(z, z^{\prime}\right)$ satisfies condition G2. Finally we need to see that $z \rightarrow G^{0, \lambda}\left(z, z^{\prime}\right)$ is a multivalued solution to the Dirac equation in $\mathbf{R}^{2} \backslash\left\{0, z^{\prime}\right\}$ with monodromy $e^{-2 \pi l \lambda}$. For definiteness fix $z^{\prime}=z_{0}^{\prime}$ in the upper half plane. Then formula (2.25) shows that $G^{0, \lambda}\left(z, z_{0}^{\prime}\right)$ is given by $G\left(z-z_{0}^{\prime}\right)+\Delta\left(z, z_{0}^{\prime}\right)$ for all 
$z$ with $\arg (z) \in\left(0, \arg \left(z_{0}^{\prime}\right)+\pi\right)$. For $\arg (z) \in\left(\arg \left(z_{0}^{\prime}\right)+\pi, 2 \pi\right)$ the formula for $G^{0, \lambda}\left(z, z_{0}^{\prime}\right)$ is $e^{-2 \pi i \lambda} G\left(z-z_{0}^{\prime}\right)+\Delta\left(z, z_{0}^{\prime}\right)$. There is an apparent "singularity" in $G^{0, \lambda}\left(z, z_{0}^{\prime}\right)$ when $\arg (z)=\arg \left(z_{0}^{\prime}\right)+\pi$ that is due to a genuine singularity in $\Delta\left(z, z_{0}^{\prime}\right)$. Formula (2.24) for $\Delta\left(z, z_{0}^{\prime}\right)$ does not make sense when $z$ lies along the ray $-z_{0}^{\prime} \mathbf{R}^{+}$, since the kernel $(u+v)^{-1}$ will then have a singularity. To study what happens as $z$ approaches this ray from the side with smaller argument one can fix the contour $z \mathbf{R}^{+}$in (2.24) to $-e^{-i \delta} z_{0}^{\prime} \mathbf{R}^{+}$for some small $\delta>0$. In a similar fashion to study what happens for $z$ near the ray $-z_{0}^{\prime} \mathbf{R}^{+}$on the side with larger argument one can fix the ray $z \mathbf{R}^{+}$to $-e^{i \delta} z_{0}^{\prime} \mathbf{R}^{+}$. A simple residue calculation allows one to compare the two different expressions for $G^{0, \lambda}\left(z, z_{0}^{\prime}\right)$ by deforming the contour $-e^{-i \delta} z_{0}^{\prime} \mathbf{R}^{+}$to $-e^{i \delta} z_{0}^{\prime} \mathbf{R}^{+}$. One finds that $G^{0, \lambda}\left(z, z_{0}^{\prime}\right)$ is smooth across this apparent singularity. It is clear from the formulas for $G^{0, \lambda}\left(z, z_{0}^{\prime}\right)$ that the upper and lower boundary values on the positive real axis extend across the axis as smooth solutions to the Dirac equation. It remains to check that the upper and lower boundary values differ by the factor $e^{-2 \pi i \lambda}$. This is obvious for the terms involving the unperturbed Green function $G\left(z-z_{0}^{\prime}\right)$. For $\Delta\left(z, z_{0}^{\prime}\right)$ observe that as the contour $z \mathbf{R}^{+}$ approaches the positive real axis from below the principal value of the integrand $u^{-\lambda}$ will pick up an additional factor of $e^{-2 \pi i \lambda}$ compared to its boundary value from above. This finishes the verification of condition G1 for $z_{0}^{\prime}$ in the upper half plane. The same calculations can be done to check $\mathrm{G} 1$ when $z_{0}^{\prime}$ lies on the negative real axis or in the lower half plane. We have then:

THEOREM 2.1. Formulas (2.26) and (2.13) and (2.14) represent the Green function for $D_{0, \lambda}(m)$ in the sense of conditions $\mathbf{G} i, i=$ $1,2,3$.

\section{Existence for the $n$-point Green function.}

Reduction to a subspace problem. In this section we will prove the existence of a Green function $G^{a, \lambda}\left(z, z^{\prime}\right)$ for $D_{a, \lambda}(m)$ satisfying the conditions $\mathbf{G} i$ for $i=1,2,3$ when $a=\left\{a_{1}, \ldots, a_{n}\right\}$ and $\lambda=$ $\left\{\lambda_{1}, \ldots, \lambda_{n}\right\}$. For simplicity we will suppose that the branch cuts:

$$
b_{j}=\left\{z \in \mathbf{R}^{2}: z_{2}=\left(a_{j}\right)_{2} \text { and }\left(z-a_{j}\right)_{1}>0\right\}
$$

are disjoint (or what is the same thing-no two branch points have the same second coordinate). We believe that this restriction is not essential, but the modifications needed in the method we use to treat 
the existence question for $G^{a, \lambda}\left(z, z^{\prime}\right)$ when there are coincidences among the second coordinates of the $a_{j}$ are clumsy to describe and so we will not consider this case further here.

Our strategy for constructing such a Green function is standard. Suppose for the moment that such a Green function exists. Let $\varphi(z)$ denote a $C_{0}^{\infty}\left(\mathbf{R}^{2}\right)$ test function which is identically 1 in a neighborhood of 0 and which vanishes identically outside the ball of radius 1. Fix $z^{\prime}$ not on the branch cuts $b_{j}$. Then for some $\varepsilon>0$ the function $\varphi_{\varepsilon}(z):=\varphi\left(\left(z-z^{\prime}\right) / \varepsilon\right)$ will vanish identically in a neighborhood of the branch cuts $b_{j}$. A simple calculation using G3 shows that for $z \in \mathbf{R}^{2} \backslash b$ we have:

$$
\begin{array}{r}
D(m)_{z}\left(G^{a, \lambda}\left(z, z^{\prime}\right)-\varphi_{\varepsilon}(z) G\left(z-z^{\prime}\right)\right) \\
=\left[\begin{array}{cc}
0 & \partial_{z} \varphi_{\varepsilon}(z) \\
\bar{\partial}_{z} \varphi_{\varepsilon}(z) & 0
\end{array}\right] G\left(z-z^{\prime}\right) .
\end{array}
$$

Because the derivatives of $\varphi_{\varepsilon}(z)$ vanish for $z$ near $z^{\prime}$ it follows that the columns of the matrix on the right-hand side of (3.0) are smooth functions of compact support in $z$ which vanish in a neighborhood of the union of the branch cuts $b$. To construct the Green function $G^{a, \lambda}\left(z, z^{\prime}\right)$ we will show that it is always possible to solve:

$$
D_{a, \lambda}(m) f=g
$$

for $f \in \mathscr{D}_{a, \lambda}$ whenever $g$ is a $C_{0}^{\infty}$ function whose support is disjoint from $b$. Let $g_{i}$ denote the $i$ th column of the right-hand side of (3.0) (thought of as a function of $z$ depending on the parameter $z^{\prime}$ ). Let $f_{i} \in \mathscr{D}_{a, \lambda}$ denote the solution of:

$$
D_{a, \lambda}(m)_{z} f_{i}=g_{i} .
$$

Then it is easy to check that the matrix $F\left(z, z^{\prime}\right)$ defined by:

$$
F\left(z, z^{\prime}\right)=\left[f_{1}\left(z, z^{\prime}\right), f_{2}\left(z, z^{\prime}\right)\right]+\varphi_{\varepsilon}(z) G\left(z-z^{\prime}\right)
$$

satisfies the conditions $\mathbf{G} i$ for $i=1,2,3$. The construction of the Green function $G^{a, \lambda}\left(z, z^{\prime}\right)$ is thus reduced to the solution of the equation (3.1) for right-hand side $g$ that vanishes in a neighborhood of the branch cuts $b_{j}$. We will use the following strategy to solve (3.1). Each branch cut $b_{j}$ will be isolated in an open neighborhood $S_{j}\left(b_{j} \subset\right.$ $\left.S_{j}\right)$. We will solve the unperturbed Dirac equation $D(m) f=g$ in the exterior of the union of the sets $S_{j}$ and we will show that it is possible to choose this solution so that the restriction of $f$ to the boundary $\bigcup_{j} \partial S_{j}$ has an extension into the interior of the sets $S_{j}$ which is in 
the local null space of $D_{a_{1}, \lambda}(m)$. This particular solution will be the appropriate solution to (3.1). The focus on boundary subspaces for solutions to the Dirac equation is the main technical device we employ. For a reason related to our use of the transfer matrix formalism we employ horizontal strips $S_{j}$ to isolate the branch cuts $b_{j}$ (rather than the tubular neighborhoods that would otherwise be natural). This forces some preliminary complications in our treatment that we will now deal with. We will say that $S$ is a horizontal strip in $\mathbf{R}^{2}$ if $S$ consists of all points $z$ in $\mathbf{R}^{2}$ whose second coordinates $z_{2}$ lie in an open interval $(a, b)$ with $b>a$. Suppose now that the branch cuts $b_{J}$ are all disjoint and let $S_{j}$ denote a horizontal strip containing $b_{j}$ for $j=1, \ldots, n$. Since the $b_{j}$ are disjoint we can choose the strips $S_{j}$ so that their closures, $\bar{S}_{j}$ are pairwise disjoint as well. Suppose now that $g(z)$ is a $C_{0}^{\infty}$ function on $\mathbf{R}^{2}$ with values in $\mathbf{C}^{2}$ and that the support of $g(z)$ is disjoint from the branch cuts $b_{j}$. The complication mentioned above that attends the choice of strips to isolate the branch cuts $b_{j}$ is that even though the function $g(z)$ has support disjoint from the $b_{j}$ it may not be possible to choose the strips $S_{j}$ so that the support of $g(z)$ is disjoint from all the $S_{j}$. For example, if the support of $g(z)$ contains points on the horizontal line through a branch point $a_{k}$, then it is obvious that the support of $g(z)$ will always intersect $S_{k}$. We will now address the problem this creates. Let $S_{j}^{\prime}$ denote a horizontal strip which contains the branch cut $b_{j}$ and such that the closure, $\bar{S}_{j}^{\prime}$ is contained in the strip $S_{j}$. Define the open set $S_{\text {ext }}$ by:

$$
S_{\mathrm{ext}}=\mathbf{R}^{2} \backslash \bigcup_{j} \bar{S}_{j}^{\prime}
$$

Then the sets $S_{j}$ for $j=1, \ldots, n$ and $S_{\text {ext }}$ together provide an open covering of $\mathbf{R}^{2}$. Let $\psi_{j}$ for $j=1, \ldots, n$ and $\psi_{\text {ext }}$ denote a $C^{\infty}$ partition of unity subordinate to this covering. Thus $\psi_{j}$ has support in $S_{j}$ and $\psi_{\text {ext }}$ has support in $S_{\text {ext }}$ and:

$$
\psi_{\mathrm{ext}}(z)+\sum_{j=1}^{n} \psi_{j}(z)=1 .
$$

Now suppose that $g \in C_{0}^{\infty}\left(\mathbf{R}^{2}\right)$ and suppose that the support of $g$ is disjoint from the union of the branch cuts $b$. Define $g_{j}=\psi_{j} g$ and $g_{\text {ext }}=\psi_{\text {ext }} g$. To solve $D_{a, \lambda}(m) f=g$ it would suffice to solve $D_{a, \lambda}(m) f_{j}=g_{j}$ for $j=1, \ldots, n$ and $D_{a, \lambda}(m) f_{\text {ext }}=g_{\text {ext }}$ and then add the solutions together. We will not do this. Instead we use the 
one-point Green function to solve:

$$
D_{a_{j}, \lambda_{j}} f_{j}=g_{j} \text { for } j=1, \ldots, n \text {. }
$$

The functions $f_{j}(z)$ will have the right branching behavior at $z=a_{j}$ to be locally in $\mathscr{D}_{a, \lambda}$ but for $k \neq j$ they will not have branch cuts at $a_{k}$ and so will not be in $\mathscr{D}_{a, \lambda}$ globally. We fix this by multiplying each solution $f_{j}$ to (3.4) by $\psi_{j}$. Then a little thought shows that for each $j=1, \ldots, n$ the functions $\psi_{j} f_{j} \in \mathscr{D}_{a, \lambda}$. It is now clear that to solve $D_{a, \lambda}(m) f=g$ it will suffice to solve:

$$
D_{a, \lambda}(m) F=g-D_{a, \lambda}(m) \sum_{J=1}^{n} \psi_{J} f_{J}
$$

for $F$ (where each $f_{j}$ is the solution to (3.4)). But $\psi_{J}$ is identically 1 on $S_{j}^{\prime}$ and $g=g_{j}$ on $S_{j}^{\prime}$ while $\psi_{k}$ is identically 0 on $S_{j}^{\prime}$ for $k \neq j$. Thus, the right-hand side of (3.5) vanishes identically on $S_{j}^{\prime}$ for $j=1, \ldots, n$. The use of the one-point Green function to solve (3.4) presents no serious problems and so we will leave this to the reader. We have reduced the existence of the $n$-point Green function to the problem of solving $D_{a, \lambda}(m) f=g$ for $f$ when $g$ is a $C_{0}^{\infty}$ function whose support is disjoint from the union of the strips $\bar{S}_{j}^{\prime}$ containing the branch cuts $b_{j}$. Since one family of horizontal strips containing the branch cuts $b_{j}$ is like any other we will drop the prime in $S_{j}^{\prime}$ and write $S_{j}^{\prime}=S_{j}$ henceforth, maintaining the assumption that $b_{j} \subset S_{j}$ and the condition that the closures $\bar{S}_{j}$ are pairwise disjoint. Let:

$$
S=\bigcup_{j} S_{j}
$$

denote the union of the strips $S_{j}$ and write $\partial S$ for the boundary of $S$. Let $H$ denote the Hilbert space $H^{1 / 2}(\partial S)$ - the Sobolev space of order $\frac{1}{2}$ for functions defined on the boundary $\partial S$ with values in $\mathbf{C}^{2}$. We will reduce the existence question for solutions to $D_{a, \lambda}(m) f=g$ with $g \in C_{0}^{\infty}\left(\mathbf{R}^{2} \backslash \bar{S}\right)$ to a question of transversality for two subspaces $W_{\text {ext }}$, and $W_{\text {int }}(a)$ of $H$. First we describe $W_{\text {ext }}$. Let $W_{\text {ext }}$ denote the subspace of $H^{1}\left(\mathbf{R}^{2} \backslash \bar{S}\right)$ (the Sobolev space of order 1 for functions on $\mathbf{R}^{2} \backslash \bar{S}$ with values in $\mathbf{C}^{2}$ ) which consists of functions $w$ which satisfy the Dirac equation $D(m) w(z)=0$ for $z \in \mathbf{R}^{2} \backslash \bar{S}$. The Sobolev embedding theorem shows that each element of $W_{\mathrm{ext}}$ has a boundary value in $H$. The subspace of $H$ which one obtains by restricting the elements $w \in W_{\text {ext }}$ to the boundary $\partial S$ we will also denote by $W_{\text {ext }}$. 
In a similar fashion we will identify the subspace $W_{\text {int }}(a)$ first as a space of functions on $S$ and then we will pass to boundary values. We will say that a function $w(z)$ defined on $S_{j} \backslash b_{j}$ with values in $\mathbf{C}^{2}$ is locally in the null space of $D_{a, \lambda}(\mathrm{m})$ provided that the following two conditions are met:

W1. The function $w(z)$ is a multivalued solution to the Dirac equation on $S_{j}$ branched along $b_{j}$ with monodromy $e^{-2 \pi i \lambda}$ and local expansion near $z=a_{j}$ given by:

$$
w(z)=\sum_{k>0}\left\{a_{k}(w) w_{k-\lambda}\left(z-a_{j}\right)+b_{k}(w) w_{k+\lambda}^{*}\left(z-a_{\jmath}\right)\right\} .
$$

W2. For $\varepsilon>0$ the function $w(z)$ is in the Sobolev space $H^{1}\left(S_{J} \backslash\left\{B_{\varepsilon}\left(a_{j}\right) \cup b_{j}\right\}\right)$ where $B_{\varepsilon}\left(a_{J}\right)$ is the ball of radius $\varepsilon$ about $a_{J}$.

The Sobolev embedding theorem implies that any function $w$ which is locally in the null space of $D_{a, \lambda}(m)$ on $S_{J}$ has boundary values in $H^{1 / 2}\left(\partial S_{j}\right)$. Thus we make the following definition:

Definition 3.0. We will say that $w \in W_{\text {int }}(a) \subset H$ provided that for each $j=1, \ldots, n$ the restriction $\left.w\right|_{\partial S}$ is the boundary value of a function on $S_{j}$ which is locally in the null space of $D_{a, \lambda}(m)$.

We are now prepared to state the main technical result of this section.

THEOREM 3.1. The subspaces $W_{\mathrm{ext}}$ and $W_{\mathrm{int}}(a)$ are transverse in $H$.

Before we turn to the proof of this result (which will occupy us for some time) it is useful to see how it can be used to settle the existence question for solutions to $D_{a, \lambda}(m) f=g$ where $g$ vanishes in a neighborhood of $\bar{S}_{j}$ for $j=1, \ldots, n$. First we solve $D(m) f_{0}=$ $g$ for $f_{0} \in H^{1}\left(\mathbf{R}^{2}\right)$. The restriction of $f_{0}$ to $\partial S$ is then in $H$. The transversality result allows us to split $\left.f_{0}\right|_{S}=f_{\text {ext }}+f_{\text {int }}$ where $f_{\text {ext }} \in W_{\text {ext }}$ and $f_{\text {int }} \in W_{\text {int }}(a)$. The function $f_{\text {ext }}$ may also be regarded as a function defined in the exterior of the union of the strips $S_{J}$ which satisfies the Dirac equation there. Define:

$$
f(z)=f_{0}(z)-f_{\mathrm{ext}}(z) \text { for } z \notin S .
$$

Then $f(z)$ satisfies $D(m) f(z)=g(z)$ for $z \notin S$ and the boundary value of $f$ on $\partial S$ is equal to $f_{\text {int }}$ which has a continuation into the interior of $S$ which is locally in the null space of $D_{a, \lambda}(m)$ Elliptic regularity implies that $f$ continues across $\partial S$ as a local solution to 
the Dirac equation, Proposition 1.1 implies that the extension of $f$ to $\mathbf{R}^{2} \backslash \bigcup_{j} b_{j}$ (which we continue to denote by $f$ ) is in $\mathscr{D}_{a, \lambda}$ and we have:

$$
D_{a, \lambda}(m) f=g .
$$

Thus Theorem 3.1 will imply the existence result for the Green function $G^{a, \lambda}\left(z, z^{\prime}\right)$ that we seek.

The transfer formalism. We will now turn to some preliminary developments for the proof of Theorem 3.1. What we require is the transfer matrix characterization of the subspace $W_{\mathrm{nt}}(a)$. We begin by describing a splitting of the Sobolev space $H^{1 / 2}(L)$ for any horizontal line $L$ in $\mathbf{R}^{2}$ that will be convenient to work with in describing the more elaborate splitting of $H^{1 / 2}(\partial S)$ into $W_{\mathrm{ext}}$ and $W_{\text {int }}(a)$. Let $z=x+i y$ be represented in the usual fashion in terms of horizontal and vertical coordinates $x$ and $y$. If one solves the Dirac equation $D(m) \psi=0$ for the $y$ derivative of $\psi$ one finds:

$$
\partial_{y} \psi=D_{0} \psi
$$

where:

$$
D_{0} g=\left[\begin{array}{cc}
i \partial_{x} & -\frac{i m}{2} \\
\frac{i m}{2} & -i \partial_{x}
\end{array}\right] g
$$

Let

$$
\hat{g}(\xi)=\frac{1}{2 \pi} \int_{\mathbf{R}} g(x) e^{-i x \xi} d x
$$

denote the Fourier transform. After Fourier transform the operator $D_{0}$ becomes multiplication by the matrix valued function $D_{0}(\xi)$ given by:

$$
D_{0}(\xi)=\omega(\xi)\left(Q_{-}(\xi)-Q_{+}(\xi)\right)
$$

where $\omega(\xi)=\left[\xi^{2}+m^{2}\right]^{1 / 2}$ and the functions $Q_{ \pm}(\xi)$ are defined by:

$$
Q_{ \pm}(\xi)=\left[\begin{array}{cc}
\frac{\omega(\xi) \pm \xi}{2 \omega(\xi)} & \pm \frac{i m}{2 \omega(\xi)} \\
\mp \frac{i m}{2 \omega(\xi)} & \frac{\omega(\xi) \mp \xi}{2 \omega(\xi)}
\end{array}\right] .
$$

The operators $Q_{ \pm}$on $H^{1 / 2}(\mathbf{R})$ induced by the matrix multiplication operators $Q_{ \pm}(\xi)$ in $(3.10)$ are self-adjoint projections on $H^{1 / 2}(\mathbf{R})$ with the property that $Q_{-}+Q_{+}=I$, the identity on $H^{1 / 2}(\mathbf{R})$. We define:

$$
H_{ \pm}=Q_{ \pm} H^{1 / 2}(\mathbf{R})
$$


If $L=\{(x, y) \mid y=$ constant $\}$ is a horizontal line in $\mathbf{R}^{2}$ then $L$ can be identified with $\mathbf{R}$ in an obvious fashion and $H^{1 / 2}(L)$ can be correspondingly identified with $H^{1 / 2}(\mathbf{R})$. Relative to this identification we will write:

$$
H^{1 / 2}(L)=H_{-} \oplus H_{+} .
$$

It is fairly clear from (3.7)-(3.10) that if $g \in H_{-} \subset H^{1 / 2}(L)$ then $g$ will be the boundary value of a solution to the Dirac equation which is well behaved in the half plane that lies below the line $L$, and if $g \in H_{+} \subset H^{1 / 2}(L)$ then $g$ will be the boundary value of a solution to the Dirac equation which is well behaved in the half plane that lies above the line $L$. Let $L_{-}$denote the half plane bounded above by $L$ and let $L_{+}$denote the half plane bounded below by $L$. The following proposition makes this connection between the elements of $H_{ \pm}$and solutions to the Dirac equation more precise:

Proposition 3.2. Each element $g \in H_{-} \subset H^{1 / 2}(L)$ is the boundary value of a function $g \in H^{1}\left(L_{-}\right)$which is a solution to the Dirac equation in the lower half plane $L_{-}$. Each element $g \in H_{+} \subset H^{1 / 2}(L)$ is the boundary value of a function $\mathbf{g} \in H^{1}\left(L_{+}\right)$which is a solution to the Dirac equation in the upper half plane $L_{+}$.

Proof. For the proof it will be very convenient to introduce a relative of the rational parametrization for the curve $(\xi, \omega)$ that was used in the Green function calculation in $\S 2$. Let

$$
v:=\frac{\omega+\xi}{m}
$$

so that

$$
v^{-1}=\frac{\omega-\xi}{m}
$$

and one has the rational parametrizations $\xi(v)=\frac{m}{2}\left(v-v^{-1}\right)$, and $\omega(v)=\frac{m}{2}\left(v+v^{-1}\right)$. To avoid introducing extra notation we write $\omega(\xi)=\left[\xi^{2}+m^{2}\right]^{1 / 2}$ and also $\omega(v)=\frac{m}{2}\left(v+v^{-1}\right)$-we hope that the usage will be clear in context. A simple calculation shows that:

$$
\int_{\mathbf{R}}\left\|Q_{ \pm}(\xi) \hat{g}(\xi)\right\|^{2} \omega(\xi) d \xi=\frac{m}{2} \int_{0}^{\infty}\left|g_{ \pm}(v)\right|^{2} \omega(v) \frac{d v}{v}
$$

where:

$$
g_{+}(v)=v^{1 / 2} \hat{g}_{1}(\xi(v))+i v^{-1 / 2} \hat{g}_{2}(\xi(v))
$$


and

$$
g_{-}(v)=i v^{-1 / 2} \hat{g}_{1}(\xi(v))+v^{1 / 2} \hat{g}_{2}(\xi(v)) .
$$

From (3.13) it follows that the map which takes $g$ to $\frac{m}{2}\left(g_{-}, g_{+}\right)$ maps $H^{1 / 2}(\mathbf{R})$ isometrically onto the space $L^{2}\left(\mathbf{R}_{+}, \omega(v) \frac{d v}{v}\right)$. The square roots in (3.14) and (3.15) were chosen so that the relevant measure $\omega(v) \frac{d v}{v}$ is invariant under the map $v \rightarrow v^{-1}$-a property that will be useful for us in a later calculation. The Fourier inversion formula applied to $\hat{g}$ together with the change of variables $\xi=\xi(v)$ gives:

$$
g(x)=\int_{0}^{\infty} \hat{g}(\xi(v)) e^{i x \xi(v)} \xi^{\prime}(v) d v .
$$

Solving (3.14) and (3.15) for $\hat{g}(\xi(v))$ in terms of $g_{ \pm}(v)$ one finds:

$$
g(x)=\frac{m}{2} \int_{0}^{\infty} e^{l x \xi(v)} M(v)\left[\begin{array}{l}
g_{+}(v) \\
g_{-}(v)
\end{array}\right] \frac{d v}{v}
$$

where:

$$
M(v)=\left[\begin{array}{cc}
v^{1 / 2} & -i v^{-1 / 2} \\
-i v^{-1 / 2} & v^{1 / 2}
\end{array}\right]
$$

Without loss of generality we may suppose that the line $L$ is the "real axis", that is $L=\{(x, 0) \mid x \in \mathbf{R}\}$. Suppose that $g \in H_{+}$. Then $g_{-}(v)=0$ and using (3.7) to solve the Dirac equation in the upper half plane with "initial data" $g$ on $L$ one finds the solution:

$$
\mathbf{g}(x, y)=\frac{m}{2} \int_{0}^{\infty} e^{-y \omega(v)} e^{i x \xi(v)} M(v)\left[\begin{array}{c}
g_{+}(v) \\
0
\end{array}\right] \frac{d v}{v}
$$

where $y \geq 0$. We wish to show that the $H^{1}$ norm of $\mathbf{g}$ in the upper half plane is equal to the $H^{1 / 2}$ norm of $g$ on the real line. We will do this by a Stokes' theorem calculation. Suppose that $f$ is a differentiable $\mathbf{C}^{2}$ valued function on an open subset $U$ of $\mathbf{R}^{2}$ and define a one form $\Omega(f)$ on $U$ by:

$$
\Omega(f)=i\left(\bar{f}_{1} \bar{\partial}_{z} f_{1}+\bar{f}_{2} \bar{\partial}_{z} f_{2}\right) d \bar{z}-i\left(\bar{f}_{1} \partial_{z} f_{1}+\bar{f}_{2} \partial_{z} f_{2}\right) d z .
$$

If $f$ satisfies the Dirac equation $D(m) f=0$ on $U$ then a simple calculation shows that:

$$
d \Omega(f)=\frac{1}{2}\left\{\left|\partial_{x} f\right|^{2}+\left|\partial_{y} f\right|^{2}+m^{2}|f|^{2}\right\} i d z d \bar{z} .
$$

Now let $U_{n}$ denote the rectangle in the upper half plane with vertices given by $(-n, 0),(n, 0),(n, h)$ and $(-n, h)$ for $h>0$. We wish to calculate:

$$
\int_{U_{n}} d \Omega(\mathbf{g})
$$


using Stokes' theorem and then pass to the limit $n \rightarrow \infty$. To avoid any subtlety in the last limit we will suppose to begin with that $g_{+} \in$ $C_{0}^{\infty}\left(\mathbf{R}^{+}\right)$(thus $g_{+}(v)$ vanishes in neighborhoods of $v=0$ and $v=$ $\infty)$. Stokes' theorem now implies:

$$
\int_{U_{n}} d \Omega(\mathbf{g})=\int_{\partial U_{n}} \Omega(\mathbf{g})
$$

Since $g_{+} \in C_{0}^{\infty}\left(\mathbf{R}^{+}\right)$and

$$
e^{i x \xi(v)}=\frac{2 v^{2}}{i m x\left(1+v^{2}\right)} \frac{\partial}{\partial v} e^{i x \xi(v)}
$$

we may integrate by parts repeatedly in (3.17) to see that $\mathbf{g}(x, y)$ and its derivatives tend uniformly to 0 for $y \in[0, \infty)$ as $x \rightarrow \pm \infty$. Thus the vertical pieces of the boundary of $U_{n}$ make no contribution in the limit $n \rightarrow \infty$. Let $U$ denote the horizontal strip bounded above by $y=h$ and below by $y=0$. Then since

$$
\|\mathbf{g}\|_{H^{1}(U)}^{2}=\int_{U} d \Omega(\mathbf{g})
$$

we have

$$
\|\mathbf{g}\|_{H^{1}(U)}^{2}=\int_{\partial U} \Omega(\mathbf{g}) .
$$

Now we wish to let $h \rightarrow \infty$ in (3.21). It is clear from (3.17) that the $H^{1 / 2}$ norm of $\mathbf{g}(\cdot, h)$ tends to 0 as $h \rightarrow \infty$. Since this dominates the integral of $\Omega(\mathbf{g})$ over the upper boundary $\partial_{+} U$ of $U$ it follows that in the limit $h \rightarrow \infty$ equation (3.21) becomes

$$
\|\mathbf{g}\|_{H^{1}\left(\mathbf{R}^{2}\right)}^{2}=\int_{\mathbf{R}} \Omega(\mathbf{g}) \text {. }
$$

Since $d z=d \bar{z}=d x$ on $\mathbf{R}$ and $i \bar{\partial}_{z}-i \partial_{z}=-\partial_{y}$ it follows that

$$
\int_{\mathbf{R}} \Omega(\mathbf{g})=-\int_{\mathbf{R}} \overline{\mathbf{g}} \cdot \partial_{y} \mathbf{g} d x
$$

But $\mathbf{g}$ is a solution to the Dirac equation so $\partial_{y} \mathbf{g}=D_{0} \mathbf{g}$ and since $g \in H_{+}$we have $\left.D_{0} \mathbf{g}\right|_{\mathbf{R}}=D_{0} g=-\omega g$. Thus

$$
\|\mathbf{g}\|_{H^{1}\left(\mathbf{R}^{2}\right)}^{2}=\int_{\mathbf{R}} \bar{g} \cdot \omega g d x=\|g\|_{H^{1 / 2}(\mathbf{R})}^{2} .
$$

We proved (3.23) for $g_{+} \in C_{0}^{\infty}\left(\mathbf{R}^{+}\right)$. But the functions $g$ which have such coordinates $g_{+}$are clearly dense in $H_{+}$and (3.23) then follows for all $g \in H_{+}$by taking limits. The analogous result for $H_{-}$ 
may be proved along the same lines and this finishes the proof of the proposition.

Next we will give a simple formula for the projections $Q_{-}$and $Q_{+}$ in terms of the Green function for $D(m)$.

Proposition 3.2. Suppose that $f \in H^{1 / 2}(\mathbf{R})$ with values in $\mathbf{C}^{2}$ and define:

$$
Q f(z)=\int_{\mathbf{R}} G_{\cdot, 1}\left(z-z^{\prime}\right) f_{1}\left(z^{\prime}\right) d z^{\prime}+G_{\cdot, 2}\left(z-z^{\prime}\right) f_{2}\left(z^{\prime}\right) d \bar{z}^{\prime}
$$

where $z \notin \mathbf{R}$ and $G_{., i}$ stands for the $i^{\text {th }}$ column of the matrix $G$. Then the boundary value on $\mathbf{R}$ of the restriction of $Q f(z)$ to the upper half plane is $Q_{+} f$ and the boundary value on $\mathbf{R}$ of the restriction of $Q f(z)$ to the lower half plane is $Q_{-} f$.

Proof. Suppose that $z$ is in the upper half plane and $z^{\prime}$ is on the real axis. Recall formula (2.2) for $G\left(z-z^{\prime}\right)$ in terms of $G_{0}\left(z-z^{\prime}\right)$ and formula (2.0) for $G_{0}\left(z-z^{\prime}\right)$. In the formula for $G\left(z-z^{\prime}\right)$ that results from substituting (2.0) in (2.2) we can choose the variable $u$ to be on the positive imaginary axis. Substitute $u=i v^{-1}$ where $v \in(0, \infty)$ in this formula and use the result in (3.24). Letting $z=x+i y$ one finds:

$$
Q f(z)=\frac{m}{2} \int_{0}^{\infty} e^{i x \xi(v)} e^{-y \omega(v)} M(v)\left[\begin{array}{c}
f_{+}(v) \\
0
\end{array}\right] \frac{d v}{v} .
$$

If one compares this with (3.17) above then it is clear that the upper boundary value of $Q f(z)$ is $Q_{+} f$. A precisely analogous calculation identifies the boundary value of $Q f(z)$ from below with $Q_{-} f$.

The following theorem is the principal tool that we will use in dealing with the subspace $W_{\text {int }}(a)$.

Theorem 3.3. Suppose that $S$ is a horizontal strip containing the positive real axis in its interior. Suppose that $g \in H^{1 / 2}(\partial S)$, and let $G^{0, \lambda}\left(z, z^{\prime}\right)$ denote the one-point Green function constructed in $\S 2$. Then $g \rightarrow P_{S}(\lambda) g$ defined by

$$
P_{S}(\lambda) g(z)=\int_{\partial S} G_{\cdot, 1}^{0, \lambda}\left(z, z^{\prime}\right) g_{1}\left(z^{\prime}\right) d z^{\prime}+G_{\cdot, 2}^{0, \lambda}\left(z, z^{\prime}\right) g_{2}\left(z^{\prime}\right) d \bar{z}^{\prime}
$$

for $z \in S$ is a projection onto the functions locally in the domain of $D_{0, \lambda}(m)$. The boundary $\partial S$ in the circuit integral (3.25) is positively oriented in the direction of increasing first coordinates on its bottom portion and negatively oriented on its top portion. Functions locally 
in the domain of $D_{0, \lambda}(m)$ have boundary values on $\partial S$ in $H^{1 / 2}(\partial S)$ and so by passing to boundary values (3.25) can also be interpreted as a map on $H^{1 / 2}(\partial S)$.

Proof. We will first show that $P_{S}(\lambda) g(z)$ has a convergent local expansion of type (3.6) near $z=0$. We can use the formula (2.14) for the Green function $G^{0, \lambda}$ to calculate the local expansion coefficients for $P_{S}(\lambda) g(z)$. Elementary estimates of these coefficients will show that the local expansion for $P_{S}(\lambda) g$ converges for $z$ close enough to 0 . Combining (3.16) and (2.14) one finds that the local expansion coefficient $a_{k}\left(P_{S}(\lambda) g\right)$ in the expansion (3.6) of $P_{S}(\lambda) g(z)$ near 0 is given by:

$$
\begin{aligned}
a_{k}\left(P_{S}(\lambda) g\right)= & \frac{i m(-1)^{k-1 / 2}}{4 \sin (\pi \lambda)} \int_{\partial S} \hat{w}_{k-\lambda, 1}\left(z^{\prime}\right) g_{1}\left(z^{\prime}\right) d z^{\prime} \\
& +\hat{w}_{k-\lambda, 2}\left(z^{\prime}\right) g_{2}\left(z^{\prime}\right) d \bar{z}^{\prime}
\end{aligned}
$$

We estimate this using the representation (2.19) for the wave function $\hat{w}_{l}$. It will be enough to illustrate the idea for that portion of the contour $\partial S$ which lies in the upper half plane. Suppose the upper part of $\partial S$ is $\partial_{+} S=\{(x, \varepsilon) \mid x \in \mathbf{R}$, with $\varepsilon>0$ fixed $\}$, then for $z^{\prime} \in \partial_{+} S$ we can fix the contour for the variable $v$ in (2.19) so that $v=i u$ with $u \in \mathbf{R}^{+}$. For $z^{\prime}=x+i \varepsilon$ one finds

$$
e\left(z^{\prime}, i u\right)=e^{-\varepsilon \omega(u)} e^{-i x \xi(u)}
$$

where $\omega(u)=\frac{m}{2}\left(u+u^{-1}\right)$ and $\xi(u)=\frac{m}{2}\left(u-u^{-1}\right)$. If we substitute (2.19) into the part of the contour integral in (3.17) over $\partial_{+} S$ then we find that the result is dominated by:

$$
\frac{m|\cot (\pi \lambda)|}{4 \pi} \int_{0}^{\infty} e^{-\varepsilon \omega(u)} u^{\lambda-k}\left|g_{-}(u)\right| \frac{d u}{u} .
$$

Employing the Cauchy-Schwarz inequality in (3.27) with the measure $\omega(u) \frac{d u}{u}$ and then using the inequality $u^{2 \lambda} / \omega(u)<1$ and the representation:

$$
K_{k}(x)=\int_{0}^{\infty} e^{-(x / 2)\left(u+u^{-1}\right)} u^{l} \frac{d u}{u}
$$

one finds that (3.27) is dominated by:

$$
\frac{m|\cot (\pi \lambda)|}{4 \pi}\left[K_{2 k}(2 m \varepsilon)\right]^{1 / 2}\left[\int_{0}^{\infty}\left|g_{-}(u)\right|^{2} \omega(u) \frac{d u}{u}\right]^{1 / 2} .
$$

Consulting (3.13) we see that this is in turn dominated by

$$
\frac{|\cot (\pi \lambda)|}{2 \pi}\left[K_{2 k}(2 m \varepsilon)\right]^{1 / 2}\|g\|_{1 / 2} \text {. }
$$


Finally the representation for $K_{l}$ given above makes the following estimate trivial

$$
K_{l}(x)<\left[\frac{2}{x}\right]^{l} \Gamma(l)
$$

for $l>0$. Using this last estimate in (3.28) we find that (3.27) is dominated by

$$
\frac{|\cot (\pi \lambda)|}{2 \pi(m \varepsilon)^{k}} \Gamma(2 k)^{1 / 2}\|g\|_{1 / 2}
$$

A precisely similar estimate of the contribution to the expansion coefficient

$$
a_{k}\left(P_{S}(\lambda) g\right)
$$

that comes from the lower contour $\partial_{-} S$ shows that it is also dominated by an expression of the form (3.29) (of course, $\varepsilon$ may change). If we now use the estimate (3.29) for $a_{k}\left(P_{S}(\lambda) g\right)$ Stirling's asymptotic estimate for the $\Gamma$ function, and simple estimates for Bessel functions that one may deduce from the formulas that follow (1.7) it is easy to see that the local expansion (3.6) for $P_{S}(\lambda) g(z)$ will converge absolutely for all $z$ with $|z|$ sufficiently small. Furthermore the radius about 0 at which such convergence takes place can be fixed independent of $g \in H^{1 / 2}(\partial S)$. Let $\rho>0$ denote a fixed radius at which the local expansion (3.6) converges for all $g \in H^{1 / 2}(\partial S)$. To finish the proof of this proposition it will be enough to show that $P_{S}(\lambda) g(z)$ is in $H^{1}\left(S \backslash\left\{B_{\rho}(0) \cup \mathbf{R}^{+}\right\}\right)$. We will do this by a Stokes' theorem calculation as in Proposition 3.1. For simplicity write $f(z)=P_{S}(\lambda) g(z)$ and define the one form $\Omega(f)$ as in (3.18). Of course, $f$ is multivalued across the branch cut $\mathbf{R}^{+}$but because the monodromy is unitary it is easy to see that $\Omega(f)$ is a smooth one form in $S \backslash\{0\}$. Let $U_{n}$ denote the subregion of $S$ which lies outside the ball of radius $\rho$ about 0 and inside the vertical strip $|x|<n$. To remove any subtlety in the application of Stokes' theorem suppose for the moment that $g_{ \pm} \in C_{0}^{\infty}\left(\mathbf{R}^{+}\right)$for both the restriction of $g$ to $\partial_{-} S$ and $\partial_{+} S$. Then as in (3.20) one finds

$$
\|f\|_{H^{1}\left(U_{n}\right)}^{2}=\int_{\partial U_{n}} \Omega(f) .
$$

We wish to pass to the limit $n \rightarrow \infty$ in (3.30). Formula (2.25) for the Green function $G^{0, \lambda}\left(z, z^{\prime}\right)$ and the same sort of estimates used to pass to the limit $n \rightarrow \infty$ in (3.20) show that contribution to the right-hand side of (3.30) which arises from the vertical line segments 
$x= \pm n$ tends to 0 in the limit $n \rightarrow \infty$. Let $U=S \backslash B_{\rho}(0)$. Then passing to the limit $n \rightarrow \infty$ in (3.30) one finds

$$
\|f\|_{H^{1}(U)}^{2}=\|f\|_{H^{1 / 2}(\partial S)}^{2}-\int_{\partial B_{p}(0)} \Omega(f) .
$$

The convergence of the local expansion for $f$ renders the integral of $\Omega(f)$ over $\partial B_{\rho}(0)$ finite. Thus we will know that the $H^{1}(U)$ norm of $f$ will be finite provided that the $H^{1 / 2}$ norm of the restriction of $f$ to $\partial S$ is finite. We will now formulate a proposition which shows this is true and in addition provides a useful formula for the interior boundary value of $P_{S}(\lambda) g(z)$ on $\partial S$.

Let $S$ denote a horizontal strip whose upper boundary is the horizontal line $y=y_{+}$and whose lower boundary is the horizontal line $y=y_{-}$, with $y_{-}<0<y_{+}$. Suppose that $g \in H^{1 / 2}(\partial S)$ and write $g^{U}$ for the restriction of $g$ to the upper boundary $\partial_{+} S$ and $g^{L}$ for the restriction of $g$ to the lower boundary $\partial_{-} S$. Write $g_{ \pm}^{U}(u)$ and $g_{ \pm}^{L}(u)$ for the "coordinates" of $g^{L}$ given by (3.14) and (3.15). It will be convenient to use the following representation for $g$ :

$$
g=\left[\begin{array}{l}
g_{-}^{U} \\
g_{+}^{L}
\end{array}\right] \oplus\left[\begin{array}{l}
g_{+}^{U} \\
g_{-}^{L}
\end{array}\right] .
$$

The following proposition will provide the result needed to finish the proof of Theorem 3.1 and will also be used to provide the one technical estimate needed in the proof of the main result of this section, Theorem 3.1.

Proposition 3.4. The boundary value of $P_{S}(\lambda) g(z)$ on $\partial S$ is given by

$$
P_{S} g=\left[\begin{array}{l}
g_{-}^{U} \\
g_{+}^{L}
\end{array}\right] \oplus\left[\begin{array}{ll}
\alpha & \beta \\
\gamma & \delta
\end{array}\right]\left[\begin{array}{l}
g_{-}^{U} \\
g_{+}^{L}
\end{array}\right]
$$

in the coordinate representation (3.32) and the operators $\alpha, \beta, \gamma$, and $\delta$ are given by:

$$
\begin{gathered}
\alpha g(u)=-i \frac{s_{\lambda}}{\pi} \int_{0}^{\infty} \frac{k_{\lambda}\left(u,-y_{+}\right) k_{\lambda}\left(v,-y_{+}\right)}{1+u v} g(v) \frac{d v}{v}, \\
\delta g(u)=-i \frac{s_{\lambda}}{\pi} \int_{0}^{\infty} \frac{k_{-\lambda}\left(u, y_{-}\right) k_{-\lambda}\left(v, y_{-}\right)}{1+u v} g(v) \frac{d v}{v}
\end{gathered}
$$




$$
\begin{gathered}
\beta g(u)=e^{i \pi \lambda} c_{\lambda} e^{\left(y_{-}-y_{+}\right) \omega(u)} g(u) \\
-\frac{s_{\lambda}}{\pi} \int_{0}^{\infty} \frac{k_{\lambda}\left(u,-y_{+}\right) k_{-\lambda}\left(v, y_{-}\right)}{u-v}, \\
\gamma g(u)=e^{-i \pi \lambda} c_{\lambda} e^{\left(y_{-}-y_{+}\right) \omega(u)} g(u) \\
-\frac{s_{\lambda}}{\pi} \int_{0}^{\infty} \frac{k_{-\lambda}\left(u, y_{-}\right) k_{\lambda}\left(v,-y_{+}\right)}{u-v} g(v) \frac{d v}{v},
\end{gathered}
$$

where the kernel $(u-v)^{-1}$ is understood in the principal value sense, $c_{\lambda}=\cos (\pi \lambda), s_{\lambda}=\sin (\pi \lambda)$, and $k_{\mu}(u, a)$ is defined by

$$
k_{\mu}(u, a)=u^{\mu+1 / 2} e^{a \omega(u)} .
$$

Proof. This is a fairly routine but somewhat tedious calculation involving the representation (2.25) for the Green function, and the transform (3.14), (3.15) and its inverse (3.16). We will indicate how it goes and leave the details to the reader. In the formula (3.25) suppose that both $z$ and $z^{\prime}$ both belong to $\partial_{+} S$. Then $G^{0, \lambda}\left(z, z^{\prime}\right)=$ $G\left(z-z^{\prime}\right)+\Delta\left(z, z^{\prime}\right)$ and Proposition 3.2 shows that $G\left(z-z^{\prime}\right)$ makes a contribution to (3.25) given by the first term in the direct sum on the right-hand side of (3.31). Now we concentrate on the contribution that comes from $\Delta\left(z, z^{\prime}\right)$. It is useful to notice that the matrix which appears in the definition of $\Delta\left(z, z^{\prime}\right)$ factors as follows:

$$
\left[\begin{array}{cc}
1 & -v \\
-u & u v
\end{array}\right]=\left[\begin{array}{c}
1 \\
-u
\end{array}\right]\left[\begin{array}{ll}
1 & -v
\end{array}\right]
$$

In formula (2.24) for $\Delta\left(z, z^{\prime}\right)$ one can choose both of the contours to be $i \mathbf{R}^{+}$parametrizing the integrals by $i u$ and $i v$ for $u, v \in \mathbf{R}^{+}$. Once this is done and (2.24) is substituted into (3.25) the factorization (3.38) makes it easy to recognize that the $z^{\prime}$ integration in (3.25) produces a term involving $g_{-}^{U}(v)$. The $u$ integration in the result can be matched with the appropriate inversion formula (3.16) once the substitution $u \rightarrow u^{-1}$ is made. One finds that the contribution made by $\Delta\left(z, z^{\prime}\right)$ is precisely given by the formula (3.34). The formula for $\delta$ above can be confirmed in the same fashion with the difference that the substitution $v \rightarrow v^{-1}$ allows one to identify the $z^{\prime}$ integral over $\partial_{-} S$ associated with $\Delta\left(z, z^{\prime}\right)$ and $g\left(z^{\prime}\right)$ in (3.25) with the appropriate multiple of $g_{+}^{L}(v)$.

To understand the contribution that the integral in (3.25) over $\partial_{+} S$ makes to the boundary value of $P_{S} g$ on $\partial_{-} S$ it is clear that we must deal with $G^{0, \lambda}\left(z, z^{\prime}\right)$ with $z$ in the lower half plane and $z^{\prime}$ in the upper half plane. Unfortunately the representation (2.25) for the Green 
function is rather clumsy on this score since it provides different looking formulas for $G^{0, \lambda}\left(z, z^{\prime}\right)$ depending on the difference of the arguments for $z$ and $z^{\prime}$. We can get around this as follows. When $z$ is in the lower half plane and $z^{\prime}$ is in the upper half plane then it is possible to deform the $v$-contour in (2.24) to $i \mathbf{R}^{+}$and the $u$-contour in (2.24) to $-i \mathbf{R}^{+}$. Depending on the disposition of $z$ and $z^{\prime}$ one can move either the $u$-contour first or the $v$-contour first so that one does not encounter a pole contribution from the singularity in $(u+v)^{-1}$. When this version of $\Delta\left(z, z^{\prime}\right)$ is used in (2.25) one finds two different representations for $G^{0, \lambda}\left(z, z^{\prime}\right)$ with contributions from $\Delta\left(z, z^{\prime}\right)$ distinguished by the boundary values $(u+v+0)^{-1}$ and $(u+v-0)^{-1}$ which appear as singular integral kernels in the formula for $\Delta\left(z, z^{\prime}\right)$. The average of these two formulas gives:

$$
G^{0, \lambda}\left(z, z^{\prime}\right)=e^{-i \pi \lambda} \cos (\pi \lambda) G\left(z-z^{\prime}\right)+\Delta_{p v}\left(z, z^{\prime}\right)
$$

where $\Delta_{p v}\left(z, z^{\prime}\right)$ is given by formula (2.24) with the $v$-contour along the positive imaginary axis, the $u$-contour along the negative imaginary axis and the singular kernel $(u+v)^{-1}$ understood in the principal value sense. With formula (3.39) one may proceed as above to calculate $\gamma$ above using (3.14)-(3.16).

A formula similar to (3.39) is true for $G^{0, \lambda}\left(z, z^{\prime}\right)$ when $z$ is in the upper half plane and $z^{\prime}$ is in the lower half plane. The only difference is that the factor $e^{-i \pi \lambda}$ should be replaced by $e^{i \pi \lambda}$ on the right-hand side of (3.39) and the $v$-contour should be $-i \mathbf{R}^{+}$and the $u$-contour should be $i \mathbf{R}^{+}$in the representation (2.24) for $\Delta_{p v}\left(z, z^{\prime}\right)$. The substitutions $u \rightarrow u^{-1}$ and $v \rightarrow v^{-1}$ should both be used in this final case to make the application of (3.14)-(3.16) completely straightforward. This finishes our indication of the calculations needed to prove (3.33).

We will now use Proposition 3.4 to finish the proof of Theorem 3.3. We need to show that the map which sends $g \in H^{1 / 2}(\partial S)$ into the boundary value of $P_{S}(\lambda) g$ on $\partial S$ is continuous in the $H^{1 / 2}$ norm. In view of (3.13) and (3.33) it is enough to show that the maps $\alpha, \beta, \gamma$, and $\delta$ are all continuous on $L^{2}\left(\mathbf{R}^{+}, \omega(v) \frac{d v}{v}\right)$. To see that $\alpha$ is continuous we factor $\alpha$ as follows:

$$
\alpha=e^{-y_{+} / 2 \omega}\left\{e^{y_{+} / 2 \omega} \alpha e^{y_{+} / 2 \omega}\right\} e^{-y_{+} / 2 \omega}
$$

and note the following easily confirmed observations about the composition factors. First $e^{-y_{+} / 2 \omega}$ is a continuous map from 
$L^{2}\left(\mathbf{R}^{+}, \omega(v) \frac{d v}{v}\right)$ to $L^{2}\left(\mathbf{R}^{+}, d v\right)$. Second, the kernel of:

$$
e^{y_{+} / 2 \omega} \alpha e^{y_{+} / 2 \omega}
$$

is square integrable and so represents a Schmidt class operator on $L^{2}\left(\mathbf{R}^{+} d v\right)$. Finally $e^{-y_{+} / 2 \omega}$ is a continuous map from $L^{2}\left(\mathbf{R}_{+}, d v\right)$ to $L^{2}\left(\mathbf{R}^{+}, \omega(v) \frac{d v}{v}\right)$. Together, these three observations show that $\alpha$ is a bounded operator on $L^{2}\left(\mathbf{R}^{+}, \omega(v) \frac{d v}{v}\right)$. In fact, it will be important for us that we have also shown that $\alpha$ is compact. The same argument shows that $\delta$ is a compact operator. To deal with $\beta$ we factor it as follows:

$$
\beta=e^{-y_{+} / 2 \omega}\left\{e^{y_{+} / 2 \omega} \beta e^{-y_{-} / 2 \omega}\right\} e^{y_{-} / 2 \omega} .
$$

The central factor in this representation of $\beta$ is no longer in the Schmidt class but because the principal value of $(u-v)^{-1}$ is the kernel of a bounded operator on $L^{2}\left(\mathbf{R}^{+}, d v\right)$ an obvious modification of the argument above shows that $\beta$ is a bounded operator on $L^{2}\left(\mathbf{R}^{+}, \omega(v) \frac{d v}{v}\right)$. This argument also works to show that $\gamma$ is bounded. To finish the proof of Theorem 3.3 it remains to show that if $g$ is the boundary value of a function locally in the domain of $D_{a, \lambda}(m)$ then $P_{S} g=g$. If $g$ is locally in the domain of $D_{a, \lambda}(m)$ then it is easy to see that the right-hand side of (3.25) is an exact one form except at $z^{\prime}=z$ and $z^{\prime}=0$. Stokes' theorem allows one to deform the line integral on the right-hand side of (3.25) into the sum of integrals over circles of radius $\varepsilon$ about the points $z^{\prime}=z$ and $z^{\prime}=0$. In the limit $\varepsilon \rightarrow 0$ no contribution comes from the point $z^{\prime}=0$ and one finds $g(z)$ for the contribution from the point $z^{\prime}=z$ in this limit. This finishes the proof of Theorem 3.3.

Transversality. We are now prepared to prove the main technical result of this section, the transversality of $W_{\mathrm{ext}}$ and $W_{\mathrm{int}}(a)$.

Proof (of Theorem 3.1). As at the beginning of this section let $\left\{S_{j}\right\}$ denote a disjoint collection of horizontal strips such that the $j$ th branch cut $b_{j}$ is a subset of $S_{j}$. Suppose that branch cuts $a_{j}$ are labeled so that the second coordinates of the $a_{j}$ increase as $j$ increases. Let $\partial_{+} S_{j}$ denote the upper boundary of $S_{j}$ and let $\partial_{-} S_{j}$ denote the lower boundary of $S_{j}$. For $j=2, \ldots, n$ let $\Delta y_{j}$ denote the difference of the $y$-coordinate of the lower boundary $\partial_{-} S_{j}$ minus the $y$-coordinate of the upper boundary $\partial_{+} S_{j-1}$. Thus $\Delta y_{j}$ is always positive for us. Suppose $f \in H^{1 / 2}(\partial S)$. We wish to show that there exist functions $g$ and $h$ such that $f=g+h$ with $g \in W_{\text {int }}(a)$ and 
$h \in W_{\text {ext }}$ and that such a decomposition is unique. The uniqueness is easily dealt with. Any function which is simultaneously in $W_{\text {int }}(a)$ and $W_{\text {ext }}$ is a multivalued solution to the Dirac equation globally in $L^{2}\left(\mathbf{R}^{2}\right)$ and with local expansions of type (1.5) at each of the branch points $a_{j}$. Thus Proposition 1.4 implies that this function must be 0 . Our strategy in proving the existence of the decomposition $g+h$ will be to show that the problem of finding $g$ reduces to the inversion of a Fredholm operator with index 0 whose null space can be identified with $W_{\text {int }}(a) \cap W_{\text {ext }}$. Since we have seen that the intersection of these subspaces is trivial this Fredholm operator must be invertible because it has index 0 . This will complete the existence proof.

Write:

$$
\left.f\right|_{\partial_{+} S}=f_{j}^{U}
$$

and:

$$
\left.f\right|_{\partial_{-} S_{J}}=f_{j}^{L}
$$

for the restriction of $f$ to the upper and lower boundaries of $S_{j}$. Suppose $f=g+h$ with $g \in W_{\text {int }}(a)$ and $h \in W_{\text {ext }}$ and use the notation (3.41) and (3.42) for $g$ and $h$ as well as $f$. We will now write down linear conditions on $g$ which characterize the decomposition $f=g+h$. For this purpose it will be very convenient to introduce:

$$
g_{j}:=\left[\begin{array}{l}
Q_{-} g_{j}^{U} \\
Q_{+} g_{j}^{L}
\end{array}\right]
$$

To understand why this is useful one should consult (3.32) and (3.33). One sees that $\left.g\right|_{\partial S}$, will be locally in the domain of $D_{a, \lambda}(m)$ provided that in the representation (3.32) for $\left.g\right|_{\partial S}$, one has:

$$
\left.g\right|_{\partial S_{j}}=g_{j} \oplus N_{j} g_{j}
$$

where:

$$
N_{j}=\left[\begin{array}{ll}
\alpha_{j} & \beta_{j} \\
\gamma_{j} & \delta_{j}
\end{array}\right]
$$

and $\alpha_{j}, \beta_{j}, \gamma_{j}$, and $\delta_{j}$ are the translates of the operators defined in (3.34)-(3.37) to the point $a_{j}$. Suppose that $a_{j}=m_{j}+i n_{j}$ with $m_{j}$ and $n_{j}$ real. Then one obtains the translate $\alpha_{j}$ from $\alpha$ by multiplying the kernel for $\alpha$ by $e^{i m_{j}(\xi(u)-\xi(v))}$ and replacing $y_{ \pm}$in the formula for $\alpha$ by $y_{ \pm}-n_{j}$ where in this last expression $y_{ \pm}$refers to the $y$ coordinates of $\partial_{ \pm} S_{j}$. The other translates are obtained in a similar fashion. It is easy to check that $\alpha_{j}$ and $\delta_{j}$ remain compact and $\gamma_{j}$ 
and $\beta_{j}$ remain bounded. Equation (3.44) shows that we may employ $g_{j}$ as a "free coordinate" for $g \in W_{\text {int }}(a)$. We will now express the conditions on $g_{j}$ which are imposed by the requirement that $\left.h\right|_{\partial S} \in$ $W_{\mathrm{ext}}$. The first condition is that $h_{1}^{L}$ should be the boundary value of an $H^{1}$ solution to the Dirac equation in the lower half plane bounded above by $\partial_{-} S_{1}$. We can write this condition as $Q_{+} h_{1}^{L}=0$. Or since $h_{1}^{L}=f_{1}^{L}-g_{1}^{L}$ we obtain:

$$
Q_{+} g_{1}^{L}=Q_{+} f_{1}^{L} .
$$

Next we express the condition that the functions $h_{j}^{U}$ and $h_{J+1}^{L}$ must be the boundary values of an $H^{1}$ solution to the Dirac equation in the strip bounded by $\partial_{+} S_{j}$ and $\partial_{-} S_{j+1}$ for $j=1, \ldots, n-1$. We find the conditions:

$$
Q_{+} h_{j+1}^{L}=e^{-\Delta y_{j+1} \omega} Q_{+} h_{j}^{U}
$$

and

$$
e^{-\Delta y_{j+1} \omega} Q_{-} h_{j+1}^{L}=Q_{-} h_{j}^{U} .
$$

Using the fact that $h_{j}^{U}=f_{j}^{U}-g_{j}^{U}$ and $h_{j}^{L}=f_{j}^{L}-g_{j}^{L}$ one may translate these last two conditions into:

$$
\begin{aligned}
& Q_{+} g_{j+1}^{L}-e^{-\Delta y_{j+1} \omega} Q_{+} g_{J}^{U}=Q_{+} f_{j+1}^{L}-e^{-\Delta y_{j+1} \omega} Q_{+} f_{j}^{U} \\
& Q_{-} g_{j}^{U}-e^{-\Delta y_{j+1} \omega} Q_{-} g_{j+1}^{L}=Q_{-} f_{j}^{U}-e^{-\Delta y_{j+1} \omega} Q_{-} f_{j+1}^{L}
\end{aligned}
$$

for $j=1, \ldots, n-1$. Finally $h_{n}^{U}$ should be the boundary value of an $H^{1}$ solution of the Dirac equation in the upper half plane bounded below by $\partial_{+} S_{n}$. Thus $Q_{-} h_{n}^{U}=0$ or:

$$
Q_{-} g_{n}^{U}=Q_{-} f_{n}^{U} \text {. }
$$

We will now use (3.44) to transform (3.46)-(3.49) into a system of equations for $g_{1}, \ldots, g_{n}$. We need to eliminate $Q_{+} g_{j}^{U}$ and $Q_{-} g_{j}^{L}$ in favor of $g_{j}$ using (3.44). One finds:

$$
Q_{+} g_{j}^{U}=\left[\begin{array}{ll}
1 & 0 \\
0 & 0
\end{array}\right] N_{j} g_{j}=\left[\begin{array}{cc}
\alpha_{j} & \beta_{J} \\
0 & 0
\end{array}\right] g_{j}
$$

and

$$
Q_{-} g_{j}^{L}=\left[\begin{array}{ll}
0 & 0 \\
0 & 1
\end{array}\right] N_{j} g_{j}=\left[\begin{array}{cc}
0 & 0 \\
\gamma_{j} & \delta_{j}
\end{array}\right] g_{j}
$$

We now wish to combine (3.47) with $j=k-1$ and (3.48) with $j=k$ for $k=2, \ldots, n-1$. It will not be important for us to keep track of the right-hand side of the resulting equations and so to unburden the 
notation we will write $F_{k}(f)$ to denote computable combinations of the functions $f_{j}=\left.f\right|_{\partial S}$, One finds combining (3.46), (3.47), (3.50) and (3.51):

$$
L_{k} g_{k-1}+g_{k}+U_{k} g_{k+1}=F_{k}(f)
$$

where

$$
L_{k}=-\left[\begin{array}{cc}
0 & 0 \\
e^{-\Delta y_{k} \omega} \alpha_{k-1} & e^{-\Delta y_{k} \omega} \beta_{k-1}
\end{array}\right]
$$

and

$$
U_{k}=-\left[\begin{array}{cc}
e^{-\Delta y_{k+1} \omega} \gamma_{k+1} & e^{-\Delta y_{k+1} \omega} \delta_{k+1} \\
0 & 0
\end{array}\right] .
$$

The reader should keep in mind that there is a difference in the matrix decomposition of (3.50) and (3.51) compared with (3.52) that accounts for the apparent switch in (3.53) and (3.54). Equations (3.52) for $k=2, \ldots, n-1$ are to be supplemented by the combinations that arise at the ends. Without difficulty the reader may verify that (3.46) and (3.49) together with the endpoint cases of (3.47) and (3.48) imply:

$$
g_{1}+U_{1} g_{2}=F_{1}(f)
$$

and:

$$
L_{n} g_{n-1}+g_{n}=F_{n}(f) .
$$

We now combine (3.52), (3.55), and (3.56) into the single matrix equation:

$$
\left[\begin{array}{ccccc}
I & U_{1} & 0 & \cdot & 0 \\
L_{2} & I & U_{2} & \cdot & 0 \\
\cdot & \cdot & \cdot & \cdot & \cdot \\
0 & \cdot & L_{n-1} & I & U_{n-1} \\
0 & \cdot & \cdot & L_{n} & I
\end{array}\right]\left[\begin{array}{c}
g_{1} \\
\cdot \\
\cdot \\
\cdot \\
g_{n}
\end{array}\right]=\left[\begin{array}{c}
F_{1}(f) \\
\cdot \\
\cdot \\
\cdot \\
F_{n}(f)
\end{array}\right]
$$

We will now show that the operator on the left-hand side of (3.57) is Fredholm with index 0 . To see this it is useful to introduce:

$$
L_{k}^{0}=-\left[\begin{array}{cc}
0 & 0 \\
0 & e^{-\Delta y_{k} \omega} \beta_{k-1}
\end{array}\right]
$$

and

$$
U_{k}^{0}=-\left[\begin{array}{cc}
e^{-\Delta y_{k+1} \omega} \gamma_{k+1} & 0 \\
0 & 0
\end{array}\right] .
$$


The matrix one obtains from the left-hand side of (3.57) by replacing $L_{k}$ by $L_{k}^{0}$ and $U_{k}$ by $U_{k}^{0}$ is:

$$
\left[\begin{array}{ccccc}
I & U_{1}^{0} & 0 & \cdot & 0 \\
L_{2}^{0} & I & U_{2}^{0} & \cdot & 0 \\
\cdot & \cdot & \cdot & \cdot & \cdot \\
0 & \cdot & L_{n-1}^{0} & I & U_{n-1}^{0} \\
0 & \cdot & 0 & L_{n}^{0} & I
\end{array}\right] .
$$

But it is easy to check that this matrix is the product of the invertible lower triangular matrix:

$$
\left[\begin{array}{ccccc}
I & 0 & \cdot & \cdot & 0 \\
L_{2}^{0} & I & 0 & \cdot & 0 \\
\cdot & \cdot & \cdot & \cdot & \cdot \\
0 & \cdot & L_{n-1}^{0} & I & 0 \\
0 & \cdot & \cdot & L_{n}^{0} & I
\end{array}\right]
$$

and the invertible upper triangular matrix:

$$
\left[\begin{array}{ccccc}
I & U_{1}^{0} & 0 & \cdot & 0 \\
0 & I & U_{2}^{0} & \cdot & 0 \\
\cdot & \cdot & \cdot & \cdot & \cdot \\
0 & \cdot & 0 & I & U_{n-1}^{0} \\
0 & \cdot & \cdot & 0 & I
\end{array}\right] .
$$

Thus (3.60) is invertible and since $\alpha_{k}$ and $\delta_{k}$ are compact the operator on the left-hand side of (3.57) is a compact perturbation of an invertible operator and hence Fredholm with index 0 . It is clear that if the operator:

$$
\left[\begin{array}{ccccc}
I & U_{1} & 0 & \cdot & 0 \\
L_{2} & I & U_{2} & \cdot & 0 \\
\cdot & \cdot & \cdot & \cdot & \cdot \\
0 & \cdot & L_{n-1} & I & U_{n-1} \\
0 & \cdot & \cdot & L_{n} & I
\end{array}\right]
$$

had a non trivial null vector this null vector could be used to construct a non trivial $g \in W_{\text {int }}(a) \cap W_{\text {ext }}$. Thus (3.61) has only a trivial null space and being a Fredholm operator with index 0 it must be invertible. This finishes the proof of Theorem (3.0).

The derivative of $G^{a, \lambda}\left(z, z^{\prime}\right)$. As a prelude to the calculation of the derivative of the $n$-point Green function $G^{a, \lambda}\left(z, z^{\prime}\right)$ with respect to the parameter $a$ we will next do a calculation that will permit us to identify the lowest order coefficients $\alpha_{1 / 2, i}^{\nu}(z)$ and $\beta_{1 / 2, i}^{\nu}(z)$ in 
the expansions (2.6) of the $n$-point Green function (the idea for this calculation can be found in SMJ III, see (3.2.11) in [14-III]). Let $f\left(z^{\prime}\right)$ denote a multivalued solution to the Dirac equation square integrable at infinity and with local expansions:

$$
f\left(z^{\prime}\right)=\sum_{k}\left\{a_{k}^{\nu} w_{k+\lambda_{\nu}}\left(z^{\prime}-a_{\nu}\right)+b_{k}^{\nu} w_{k-\lambda_{\nu}}^{*}\left(z^{\prime}-a_{\nu}\right)\right\}
$$

where we suppose that only a finite number of the coefficients $\left\{a_{k}^{\nu}, b_{k}^{\nu}\right\}$ with half integers $k<0$ are non zero.

Suppose that $w$ and $u$ are two branched solutions to the Euclidean Dirac equation with the same (unitary) monodromy at the points $a_{\nu}$. Then away from the branch points the functions $w_{j} \bar{u}_{k}$ are smooth functions and one may easily check that for $z^{\prime} \neq a_{\nu}$ :

$$
\begin{aligned}
w\left(z^{\prime}\right) \cdot \bar{u}\left(z^{\prime}\right) i d z^{\prime} d \bar{z}^{\prime} & =d\left\{\frac{2}{m} w_{2}\left(z^{\prime}\right) \bar{u}_{1}\left(z^{\prime}\right) i, d \bar{z}^{\prime}\right\} \\
& =-d\left\{\frac{2}{m} w_{1}\left(z^{\prime}\right) \bar{u}_{2}\left(z^{\prime}\right) i d z^{\prime}\right\}
\end{aligned}
$$

(see (1.12)). Now let $D_{\varepsilon}(a)$ denote the disk of radius $\varepsilon$ about the point $a$ and let $C_{\varepsilon}(a)$ denote the circle of radius $\varepsilon$ about the point $a$. Let $D_{\varepsilon}$ denote the union of the disks $D_{\varepsilon}\left(a_{\nu}\right)$ for $\nu=1, \ldots, n$ and the disk $D_{\varepsilon}(z)$. Then Stokes' theorem implies that the integral:

$$
\frac{m}{2} \int_{\mathbf{R}^{2} \backslash D_{\varepsilon}} G_{i, .}^{a, \lambda}\left(z, z^{\prime}\right) \cdot \overline{f\left(z^{\prime}\right)} i d z^{\prime} d \bar{z}^{\prime}
$$

is given by:

$$
-\sum_{\nu=1}^{n} \int_{C_{\varepsilon}\left(a_{\nu}\right)} G_{i 2}\left(z, z^{\prime}\right) \overline{f_{1}\left(z^{\prime}\right)} i d \bar{z}^{\prime}-\int_{C_{\varepsilon}(z)} G_{i 2}\left(z, z^{\prime}\right) \overline{f_{1}\left(z^{\prime}\right)} i d \bar{z}^{\prime}
$$

and also by:

$$
\sum_{\nu=1}^{n} \int_{C_{\varepsilon}\left(a_{\nu}\right)} G_{i 1}\left(z, z^{\prime}\right) \overline{f_{2}\left(z^{\prime}\right)} i d z^{\prime}+\int_{C_{\varepsilon}(z)} G_{i 1}\left(z, z^{\prime}\right) \overline{f_{2}\left(z^{\prime}\right)} i d z^{\prime} .
$$

Next we will use the local expansions for the Green function and $f\left(z^{\prime}\right)$ near the branch cuts and the asymptotics of the Green function near the diagonal $z=z^{\prime}$ to evaluate each of the last two expressions in the limit $\varepsilon \rightarrow 0$. Equating the two results will give us what we desire. Using the fact that $I_{k}(m \varepsilon)$ is $O\left(\varepsilon^{k}\right)$ and

$$
\lim _{\varepsilon \rightarrow 0} \varepsilon I_{k-1}(m \varepsilon) I_{-k}(m \varepsilon)=\frac{2 \sin (\pi k)}{m \pi}
$$


one finds that:

$$
\begin{aligned}
\lim _{\varepsilon \rightarrow 0} & \sum_{\nu=1}^{n} \int_{C_{\varepsilon}\left(a_{\nu}\right)} G_{i 2}\left(z, z^{\prime}\right) \overline{f_{1}\left(z^{\prime}\right)} i d \bar{z}^{\prime} \\
\quad & =\frac{4}{m} \sum_{\nu=1}^{n} \sum_{k>0}(-1)^{k-1 / 2} \sin \left(\pi \lambda_{\nu}\right) \beta_{k l}^{\nu}(z) \bar{a}_{-k}^{\nu}
\end{aligned}
$$

and

$$
\begin{aligned}
\lim _{\varepsilon \rightarrow 0} & \sum_{\nu=1}^{n} \int_{C_{\varepsilon}\left(a_{\nu}\right)} G_{i 1}\left(z, z^{\prime}\right) \overline{f_{2}\left(z^{\prime}\right)} i d z^{\prime} \\
& =\frac{4}{m} \sum_{\nu=1}^{n} \sum_{k>0}(-1)^{k-1 / 2} \sin \left(\pi \lambda_{\nu}\right) \alpha_{k i}^{\nu}(z) \bar{b}_{-k}^{\nu} .
\end{aligned}
$$

Each of the $k$ summations is finite because of our assumptions regarding the local expansions for $f\left(z^{\prime}\right)$.

Now one may use the fact that $K_{0}(z)$ is asymptotic to $-\log (z)$ and $K_{0}^{\prime}(z)$ is asymptotic to $-\frac{1}{z}$ as $z \rightarrow 0$ to calculate:

$$
\lim _{\varepsilon \rightarrow 0} \int_{C_{\varepsilon}(z)} G_{i 2}\left(z, z^{\prime}\right) \overline{f_{1}\left(z^{\prime}\right)} i d \bar{z}^{\prime}=i \delta_{l 2} \bar{f}_{1}(z)
$$

and

$$
\lim _{\varepsilon \rightarrow 0} \int_{C_{\varepsilon}(z)} G_{i 1}\left(z, z^{\prime}\right) \overline{f_{2}\left(z^{\prime}\right)} i d z^{\prime}=i \delta_{i 1} \bar{f}_{2}(z) .
$$

Combining these results one finds:

$$
\begin{gathered}
\sum_{\nu=1}^{n} \sum_{k>0}(-1)^{k-1 / 2} \sin \left(\pi \lambda_{\nu}\right)\left\{\beta_{k l}^{\nu}(z) \bar{a}_{-k}^{\nu}+\alpha_{k i}^{\nu}(z) \bar{b}_{-k}^{\nu}\right\} \\
=-\frac{i m}{4}\left(\delta_{i 2} \bar{f}_{1}(z)+\delta_{i 1} \bar{f}_{2}(z)\right) .
\end{gathered}
$$

We may now isolate the lowest order coefficients in the local expansion of the Green function by choosing the functions $f(z)$ appropriately. Let $W_{j}(z, \lambda)$ denote the multivalued solution to the Dirac equation which is square integrable at infinity and whose local expansion at $z=a_{\nu}$ is:

$$
W_{j}(z, \lambda)=\delta_{\nu j} w_{-1 / 2+\lambda_{\nu}}+\sum_{k>0}\left\{a_{k j}^{\nu}(\lambda) w_{k+\lambda_{\nu}}+b_{k j}^{\nu}(\lambda) w_{k-\lambda_{\nu}}^{*}\right\}
$$

where we have used the abbreviation $w_{k+\lambda_{\nu}}=w_{k+\lambda_{\nu}}\left(z-a_{\nu}\right)$ etc. Define $W_{j}^{*}(z, \lambda)$ by:

$$
W_{j}^{*}(z, \lambda)=\left(\begin{array}{l}
\bar{W}_{j 2}(z, \lambda) \\
\bar{W}_{j 1}(z, \lambda)
\end{array}\right)
$$


so that the local expansion for $W_{j}^{*}(z, \lambda)$ at $z=a_{\nu}$ is:

$$
W_{j}^{*}(z, \lambda)=\delta_{\nu j} w_{-1 / 2+\lambda_{\nu}}^{*}+\sum_{k>0}\left\{\bar{a}_{k j}^{\nu}(\lambda) w_{k+\lambda_{\nu}}^{*}+\bar{b}_{k j}^{\nu}(\lambda) w_{k-\lambda_{\nu}}\right\} .
$$

The existence of these "wave functions" is guaranteed by the SMJ results for the existence of a "canonical basis" of $L^{2}$ wave functions, even though $W_{j}(z, \lambda)$ and $W_{j}^{*}(z, \lambda)$ are not always locally in $L^{2}$ near $a_{j}$. We will spell out the connection between $W_{j}(z, \lambda)$ and the canonical basis of SMJ III in the final section of this paper which deals with the deformation analysis of the $\tau$-function. Substituting $W_{j}^{*}(z,-\lambda)$ for $f(z)$ one finds:

$$
\left(\begin{array}{c}
\alpha_{\frac{1}{2} 1}^{j}(z) \\
\alpha_{\frac{1}{2} 2}^{j}(z)
\end{array}\right)=-\frac{i m}{4 \sin \left(\pi \lambda_{j}\right)} W_{j}(z,-\lambda)
$$

Substituting $W_{j}(z, \lambda)$ for $f(z)$ one finds:

$$
\left(\begin{array}{c}
\beta_{\frac{1}{2} 1}^{j}(z) \\
\beta_{\frac{1}{2} 2}^{j}(z)
\end{array}\right)=-\frac{i m}{4 \sin \left(\pi \lambda_{j}\right)} W_{j}^{*}(z, \lambda) .
$$

We can use this result to calculate the derivative of the Green function with respect to the parameters $a_{j}$. Let $\partial_{a_{j}}:=\partial / \partial a_{j}$ denote the holomorphic and $\bar{\partial}_{a_{j}}:=\partial / \partial \bar{a}_{j}$ the antiholomorphic derivative with respect to $a_{j}$. Differentiating the local expansions (2.6) for the Green function using:

$$
\partial w_{l}=\frac{m}{2} w_{l-1}, \quad \bar{\partial} w_{l}=\frac{m}{2} w_{l+1}
$$

and

$$
\partial w_{l}^{*}=\frac{m}{2} w_{l+1}^{*}, \quad \bar{\partial} w_{l}^{*}=\frac{m}{2} w_{l-1}^{*}
$$

one finds:

$$
\partial_{a_{j}} G_{i, \lambda}^{a, \lambda}\left(z, z^{\prime}\right)=-\frac{m}{2} \delta_{j \nu} \alpha_{i / 2}^{\nu}(z) w_{-1 / 2+\lambda_{\nu}}+\text { higher order terms. }
$$

The higher order terms all involve $w_{k+\lambda_{\nu}}$ and $w_{k-\lambda_{\nu}}^{*}$ with $k>0$. In these circumstances Proposition 1.4 shows that the single leading coefficient suffices to determine the (multivalued) solution $z^{\prime} \rightarrow$ $\partial_{a} G_{i, .}^{a, \lambda}\left(z, z^{\prime}\right)$ to the Dirac equation and we find:

$$
\partial_{a,} G_{i, .}^{a, \lambda}\left(z, z^{\prime}\right)=-\frac{m}{2} \alpha_{1 / 2, i}^{j}(z) W_{j}\left(z^{\prime}, \lambda\right) .
$$


Putting this together with the previous result for $\alpha_{\frac{1}{2} i}^{j}(z)$ one finds:

$$
\partial_{a_{j}} G^{a, \lambda}\left(z, z^{\prime}\right)=\frac{i m^{2}}{8 \sin \left(\pi \lambda_{j}\right)} W_{j}(z,-\lambda) \otimes W_{j}\left(z^{\prime}, \lambda\right) .
$$

An analogous argument shows that:

$$
\bar{\partial}_{a_{j}} G^{a, \lambda}\left(z, z^{\prime}\right)=\frac{i m^{2}}{8 \sin \left(\pi \lambda_{j}\right)} W_{j}^{*}(z, \lambda) \otimes W_{j}^{*}\left(z^{\prime},-\lambda\right) .
$$

The reader might want to compare this result with (3.3.53) in SMJ III. In our formulation these derivative formulas for the Green function will provide the connection between the $\tau$-function and the local expansion coefficients for the wave functions $W_{j}(z, \pm \lambda)$ and $W_{j}^{*}(z, \pm \lambda)$ (see (4.3)). Thus (3.62) and (3.63) are the fundamental link which relates the $\tau$-functions with the deformation theory in our version of the SMJ analysis.

4. The tau function for $D_{a, \lambda}(m)$. In this section we will define the tau function for $D_{a, \lambda}(m)$ by localizing this operator away from the singularities on the branch cuts. This localized family of operators is associated with a family of subspaces in a Grassmannian affiliated with the Hilbert space of the boundary of the localization. We trivialize the det $^{*}$ bundle over this family of subspaces to define the determinant of $D_{a, \lambda}(m)$ which will be the $\tau$-function. We then establish the formula which expressed the logarithmic derivative of the $\tau$-function in terms of the local expansion coefficients of the wave functions $W_{j}(\lambda)$ and $W_{j}^{*}(\lambda)$. This is the main result of this paper. The reason for regarding the procedure we have just described as defining a determinant for $D_{a, \lambda}(m)$ is described in some detail in the first section of [8] (for the case of Cauchy-Riemann operators rather than Dirac operators but the result connecting determinant bundles with det $^{*}$ bundles remains the same). We will briefly recall this in the subsection below which deals with the det* $^{*}$ formalism. Before we do this however we will motivate the definition of the $\tau$-function with a heuristic calculation of the determinant of $D_{a, \lambda}(m)$ that we hope the reader will find instructive. After presenting this "derivation" we will show that the Grassmannian formulatiom can be used to give a rigorous definition of the $\tau$-function which leads to the same result.

$A$ heuristic calculation of $\operatorname{det}\left(D_{a, \lambda}(m)\right)$. In order to define the tau function for $D_{a, \lambda}(m)$ we wish to "regularize" the logarithmic deriva- 
tive:

$$
\begin{aligned}
d_{a} \log \operatorname{det} D_{a, \lambda}(m) & =\operatorname{Tr}\left(D_{a, \lambda}(m)^{-1} d_{a} D_{a, \lambda}(m)\right) \\
& =-\operatorname{Tr}\left(d_{a}\left(D_{a, \lambda}(m)^{-1}\right) D_{a, \lambda}(m)\right) .
\end{aligned}
$$

Let $G^{a, \lambda}\left(z, z^{\prime}\right)$ denote the Green function for $D_{a, \lambda}(m)$. Then we have seen that:

$$
\partial_{a_{\nu}} G^{a, \lambda}\left(z, z^{\prime}\right)=\frac{i m^{2}}{8 \sin \left(\pi \lambda_{\nu}\right)} W_{\nu}(z,-\lambda) \otimes W_{\nu}\left(z^{\prime}, \lambda\right)
$$

and

$$
\bar{\partial}_{a_{\nu}} G^{a, \lambda}\left(z, z^{\prime}\right)=\frac{i m^{2}}{8 \sin \left(\pi \lambda_{\nu}\right)} W_{\nu}^{*}(z, \lambda) \otimes W_{\nu}^{*}\left(z^{\prime},-\lambda\right)
$$

where $W_{\nu}$ is characterized by the local expansions:

$$
W_{\nu}(\lambda)=\delta_{j \nu} w_{-1 / 2+\lambda_{J}}+\sum_{k>0}\left\{a_{k \nu}^{j}(\lambda) w_{k+\lambda_{j}}+b_{k \nu}^{j}(\lambda) w_{k-\lambda_{j}}^{*}\right\}
$$

about the points $z=a_{j}$. Composing the operator $\partial_{a_{\nu}} G^{a, \lambda}$ with $D_{a, \lambda}(m)$ one finds the operator:

$$
\begin{aligned}
f \rightarrow & \frac{i m^{2}}{8 \sin \left(\pi \lambda_{\nu}\right)} W_{\nu}(z,-\lambda) \\
& \cdot \int_{\mathbf{R}^{2}} W_{\nu}\left(z^{\prime}, \lambda\right) \cdot J D_{a, \lambda}(m) f(z) i d z^{\prime} d \bar{z}^{\prime} .
\end{aligned}
$$

We will make sense of this operator and of the tau function trace by "localizing" the calculation away from the singularities at $a_{\nu}$. Let $\varepsilon$ denote a positive real number and for simplicity suppose that the second coordinates of the $a_{\nu}$ are distinct for distinct values of $\nu$. Let $S_{\nu}$ denote the horizontal strip containing $a_{\nu}$ with boundary $\partial S_{\nu}$ consisting of the horizontal lines with second coordinates $\pm \varepsilon$ away from the second coordinate of $a_{\nu}$. Choose $\varepsilon$ sufficiently small so that the strips $S_{\nu}$ do not intersect. In what follows one can permit the $a_{\nu}$ to have coincident second coordinates. In such circumstances one should fix branch cuts emanating from the points $a_{\nu}$ so that they do not intersect and the strips $S_{\nu}$ should be replaced by tubular neighborhoods of the branch cuts that do not intersect one another. We write

$$
S=\bigcup_{\nu} S_{\nu}
$$

To localize away from the branch cuts consider a function $f \in \mathscr{D}_{a, \lambda}$ with $D_{a, \lambda}(m) f(z)=0$ for $z \in S$. The map (4.0) induces a map on 
the boundary values of such functions on $S$ in the following manner. Let $S^{\prime}$ denote the complement of the union of the strips $S_{\nu}$. Then:

$$
\begin{aligned}
\int_{\mathbf{R}^{2}} & W_{\nu}\left(z^{\prime}, \lambda\right) \cdot J D_{a, \lambda}(m) f\left(z^{\prime}\right) i d z^{\prime} d \bar{z}^{\prime} \\
& =\int_{S^{\prime}} W_{\nu}\left(z^{\prime}, \lambda\right) \cdot J D_{a, \lambda}(m) f\left(z^{\prime}\right) i d z^{\prime} d \bar{z}^{\prime}
\end{aligned}
$$

and Stokes' theorem (see (2.7)) implies that this last integral is:

$$
\sum_{j=1}^{n} \int_{\partial S_{j}}\left\{W_{\nu, 1}\left(z^{\prime}, \lambda\right) f_{1}\left(z^{\prime}\right) d z^{\prime}+W_{\nu, 2}\left(z^{\prime}, \lambda\right) f_{2}\left(z^{\prime}\right) d \bar{z}^{\prime}\right\}
$$

where the boundaries of the strips $S_{J}$ are positively oriented on the bottom and negatively oriented on the top. If we now compare (4.0) wth (4.1) we see that the map (4.0) induces a map on functions defined on $\partial S$ obtained by restricting the output of (4.0) to $\partial S$. In order to define a trace for this map (we ignore the behavior in the interior since the "differential operator" we are looking at does not change there) we first make the observation that the boundary values of the functions $f$ on the upper $\partial_{+} S_{\nu}$ and the lower $\partial_{-} S_{\nu}$ boundaries of the strip $S_{\nu}$ are not independent of one another. Since $D_{a, \lambda}(m) f(z)=0$ inside the strip $S_{\nu}$ these upper and lower boundary values are the restriction to the boundary of a single function defined in the strip and locally in the null space of $D_{a, \lambda}(m)$. One may define a projection on this family of functions in the following way. Let $G^{\nu}\left(z, z^{\prime}\right)=G^{a_{\nu}}, \lambda_{\nu}\left(z, z^{\prime}\right)$ denote the Green function for $D_{a_{\nu}, \lambda_{\nu}}(m)$, the Dirac operator with a single branch point at $a_{\nu}$ and monodromy parameter $\lambda_{\nu}$. Suppose $f \in$ $H^{1 / 2}\left(\partial S_{\nu}\right)$. Let $G_{i k}^{\nu}\left(z, z^{\prime}\right)$ denote the matrix of the one-point Green function defined in $\S 2$. For $z$ in the interior of the strip $S_{\nu}$ define:

$$
\left(P_{\nu} f\right)_{j}(z)=\int_{\partial S_{\nu}} G_{j 1}^{\nu}\left(z, z^{\prime}\right) f_{1}\left(z^{\prime}\right) d z^{\prime}+G_{j 2}^{\nu}\left(z, z^{\prime}\right) f_{2}\left(z^{\prime}\right) d \bar{z}^{\prime} .
$$

Then as shown in Theorem 3.3 the map:

$$
\left.f \rightarrow P_{\nu} f\right|_{\partial S_{\nu}}
$$

is a projection onto the family of functions on the boundary of the strip $S_{\nu}$ which continue across the strip as elements of the null space of $D_{a_{\nu}, \lambda_{\nu}}(m)$ (and hence also as elements of the null space of: $\left.D_{a, \lambda}(m)\right)$. To make sense of the trace of (4.0) we thus compose the induced map on the boundary with the direct sum of the projections $P_{\nu}$ before taking the trace. I do not claim that the trace one gets is independent of the choice of this projection. The particular choice we 
make here is related to the business of "factoring out the one-point functions" that one finds in the representation theoretic version of the $\tau$-function in SMJ IV and SMJ V. The trace we wish to compute becomes:

$$
\begin{aligned}
-\frac{i m^{2}}{8 s_{\nu}} \sum_{j=1}^{n} \int_{\partial S_{j}}\left\{W_{\nu, 1}(z, \lambda)\right. & P_{j} W_{\nu, 1}(z,-\lambda) d z \\
& \left.+W_{\nu, 2}(z, \lambda) P_{j} W_{\nu, 2}(z,-\lambda) d \bar{z}\right\}
\end{aligned}
$$

where

$$
s_{\nu}=\sin \left(\pi \lambda_{\nu}\right)
$$

and $P_{j} W_{\nu, k}$ denotes the $k$ th component of the vector valued function $P_{j} W_{\nu}$. The local expansion for $W_{\nu}(z,-\lambda)$ in a neighborhood of $z=$ $a_{j}$ with $j \neq \nu$ shows that with the obvious restriction to $\partial S_{j}$ one has:

$$
P_{j} W_{\nu}(\cdot,-\lambda)=W_{\nu}(\cdot,-\lambda) \text { for } j \neq \nu .
$$

Because the one forms we are integrating are locally exact away from the branch points and the functions $W_{\nu}$ are exponentially small at infinity the contour integral over $\partial S_{j}$ we wish to compute for $j \neq \nu$ immediately collapses to a contour integral over a circle of small radius about $a_{j}$. A simple calculation using local expansions shows that all these integrals vanish. It remains to compute:

$$
\begin{aligned}
-\frac{i m^{2}}{8 s_{\nu}} \int_{\partial S_{\nu}}\left\{W_{\nu, 1}(z, \lambda)\right. & P_{\nu} W_{\nu, 1}(z,-\lambda) d z \\
& \left.+W_{\nu, 2}(z, \lambda) P_{\nu} W_{\nu, 2}(z,-\lambda) d \bar{z}\right\} .
\end{aligned}
$$

Once again one is integrating a locally exact one form which is well behaved at $\infty$ and so the integral collapses to a circuit integral about $a_{\nu}$ which one can evaluate using the local expansions for $W_{\nu}(z, \lambda)$ and $P_{\nu} W_{\nu}(z,-\lambda)$. Suppose the local expansion for $P_{\nu} W_{\nu}$ is

$$
P_{\nu} W_{\nu}=\sum_{k>0}\left\{f_{k} w_{k-\lambda_{\nu}}+g_{k} w_{k+\lambda_{\nu}}^{*}\right\} .
$$

Note that only expansion coefficients with $k>0$ can appear since $P_{\nu} W_{\nu}$ is locally in the null space of $D_{\nu}$. One finds

$$
\begin{aligned}
\int_{\partial S_{\nu}} & \left\{W_{\nu, 1}(z, \lambda) P_{\nu} W_{\nu, 1}(z,-\lambda) d z+W_{\nu, 2}(z, \lambda) P_{\nu} W_{\nu, 2}(z,-\lambda) d \bar{z}\right\} \\
& =\frac{4 i S_{\nu}}{m} f_{1 / 2}
\end{aligned}
$$


where

$$
s_{\nu}=\sin \left(\pi \lambda_{\nu}\right) .
$$

Using the formula for the projection $P_{\nu}$ and the formula for the onepoint Green function we compute the expansion coefficient $f_{1 / 2}$ to find:

$$
\begin{aligned}
f_{1 / 2}= & \frac{i m}{4 \sin \left(\pi \lambda_{\nu}\right)} \int_{\partial S_{\nu}} \hat{w}_{1 / 2-\lambda_{\nu}, 1}(z) W_{\nu, 1}(z,-\lambda) d z \\
& +\hat{w}_{1 / 2-\lambda_{\nu}, 2}(z) W_{\nu, 2}(z,-\lambda) d \bar{z} .
\end{aligned}
$$

Again one may collapse this contour to a small circle about the point $a_{\nu}$ and do the resulting integral using local expansions. One finds:

$$
f_{1 / 2}=a_{1 / 2, \nu}^{\nu}(-\lambda) \text {. }
$$

Putting these results together one finds:

$$
\frac{m}{2} a_{1 / 2, \nu}^{\nu}(-\lambda)
$$

for the trace of interest.

A precisely analogous calculation gives one:

$$
\frac{m}{2} \overline{a_{1 / 2, \nu}^{\nu}(\lambda)}
$$

for the $\bar{\partial}_{a_{\nu}}$ derivative of the log determinant of $D_{a, \lambda}(m)$. Our heuristic version of the tau function is thus:

$$
d_{a} \log (\tau)=\frac{m}{2} \sum_{\nu=1}^{n}\left\{a_{1 / 2, \nu}^{\nu}(-\lambda) d a_{\nu}+\overline{a_{1 / 2, \nu}^{\nu}(\lambda)} d \bar{a}_{\nu}\right\} .
$$

One could, perhaps, take (4.3) as the definition of the $\tau$-function but there are a number of objections to doing so. First one must check that the right-hand side is indeed a closed one form. The deformation theory in $\S 5$ can be used to do this (this is done in SMJ III). Secondly one would like to know that $\tau$ defined by (4.3) is a regular function of $a$ with no zeros. This is not obvious from the purely deformation theoretic point of view but it will follow from the formula for $\tau$ which we find below. Finally, (4.3) does not give any indication that the function $\tau(a)$ is the Schwinger function for a quantum field theory. This will not be evident from the formula we obtain below either but because of the link with the transfer formalism in $\S 3$ it is possible to connect simply with the scaled lattice formalism in [7] and so establish Osterwalder-Schrader positivity. 
The det* interpretation for $\tau$. Fix a choice of branch points $a_{\nu}^{0}$ for $\nu=1, \ldots, n$ without coincident second coordinates. As above let $S_{\nu}$ denote the horizontal strip of width $2 \varepsilon$ centered on the branch cut emanating from the point $a_{\nu}^{0}$. Write $S=\bigcup S_{\nu}$ and let $\partial S$ denote the boundary of $S$ oriented so that $S$ lies to the left of $\partial S$. Now suppose that for each $\nu=1, \ldots, n$ the point $a_{\nu} \in B_{\varepsilon}\left(a_{\nu}^{0}\right)$, the open ball of radius $\varepsilon$ about $a_{\nu}^{0}$. Let $W_{\text {int }}(a)$ denote the subspace of $H:=$ $H^{1 / 2}(\partial S)$ given by boundary values of functions $f \in \mathscr{D}_{a, \lambda}$ such that $D_{a, \lambda}(m) f(z)=0$ for $z$ in the interior of the set $S$ (see conditions W1 and W2 in $\S 3$ and Definition 3.1). Let $\mathscr{D}_{a, \lambda}\left(\mathbf{R}^{2} \backslash S\right)$ denote the space of $H^{1}$ functions defined in the exterior of $S$ with boundary values on $\partial S$ in the subspace $W_{\mathrm{int}}(a)$. The restriction of $D(m)$ to the domain $\mathscr{D}_{a, \lambda}\left(\mathbf{R}^{2} \backslash S\right)$ is then a natural localization of the operator $D_{a, \lambda}(m)$ to the complement of $S$ in the sense that the kernel and the cokernel of the localization can be identified with the kernel and cokernel of the full operator $D_{a, \lambda}(m)$. In fact, since the "differential operator part" of this localization of $D_{a, \lambda}(m)$ is independent of $a_{1}, \ldots, a_{n}$ (it is just $D(m)$ ) all the variation in this family of operators is captured by the subspaces $W_{\text {int }}(a)$. To define the $\tau$-function we will embed the subspaces $W_{\text {int }}(a)$ in an infinite dimensional Grassmannian of subspaces of $H$ and then trivialize the det* $^{*}$ bundle over the family $a \rightarrow W_{\text {int }}(a)$. The $\tau$-function will be obtained by comparing this trivialization with the "canonical section" of det*

To see that this is a reasonable way to define a determinant for the family of operators $D_{a, \lambda}(m)$ it will be useful to make an analogy with a somewhat less singular situation. Let $X$ denote a differential operator which is a perturbation of $D(m)$ by a $C_{0}^{\infty}$ multiplication operator with support in the set $S$. Let $\mathscr{F}$ denote the family of all such differential operators $X$. Let $W$ denote the subspace of $H$ which consists of boundary values of functions mapped to 0 in $S$ by $D(m)$ and let $V(X)$ denote the space of boundary values of functions mapped to 0 by $X$ in $S$. Let $\operatorname{Gr}(W)$ denote the Grassmannian of subspaces, $V$, of $H$ which are close to $W$ in the sense that the orthogonal projection on $W$ differs from the orthogonal projection on $V$ by a compact operator. The space $\operatorname{Gr}(W)$ is not connected and we let $\mathrm{Gr}_{0}(W)$ denote the connected component of $\operatorname{Gr}(W)$ which contains $W$. It is not difficult to see that each subspace $V(X) \in \mathrm{Gr}_{0}(W)$. Now each $X \in \mathscr{F}$ is a Fredholm map of index 0 from the domain of $D(m)$ to $L^{2}\left(\mathbf{R}^{2}\right)$ and sitting over the space of all such Fredholm maps is the determinant line bundle first defined by D. Quillen [12]. 
We will briefly describe this holomorphic line bundle. If $X$ is a Fredholm map of index 0 then there exists an invertible map $q$ such that $q^{-1} X$ is a compact perturbation of the identity. In fact it is always possible to find invertible maps $q$ such that $q^{-1} X$ is a trace class perturbation of the identity-if $q$ is such a map we will say that $q$ is an admissible parametrix for the operator $X$. The fiber in the determinant bundle over $X$ can be identified with equivalence classes of pairs $(q, \alpha)$ where $q$ is an admissible parametrix for $X$ and $\alpha$ is a complex number. Two such pairs $\left(q_{k}, \alpha_{k}\right)$ for $k=1,2$ are equivalent if

$$
\alpha_{1}=\alpha_{2} \operatorname{det}\left(q_{1}^{-1} q_{2}\right) \text {. }
$$

The multiplicative property of determinants makes it possible to check that this is indeed an equivalence relation. The map which sends $X$ into the "relative determinant", $\left(q, \operatorname{det}\left(q^{-1} X\right)\right)$, is easily seen to be a section of the determinant bundle. This section is called the canonical section and we denote it by $\sigma$. For our family $\mathscr{F}$ of Fredholm operators making a choice of a relative determinant for each element of the family is intuitively the same as finding a trivialization of the determinant bundle. If $\delta$ is a nonvanishing section of det $\rightarrow \mathscr{F}$, then we can define a determinant for the family $\mathscr{F}$ by

$$
\operatorname{det}(X)=\frac{\sigma(X)}{\delta(X)}
$$

What has this to do with Grassmannians? G. Segal and G. Wilson [15] have defined a determinant bundle over the infinite dimensional Grassmannian $\operatorname{Gr}(W)$. The fiber is a natural analogue of the highest exterior power of a subspace in finite dimensions. We write det* for the dual of this determinant bundle over $\operatorname{Gr}(W)$. A connection between the determinant bundle and the $\operatorname{det}^{*}$ bundle is provided by the following result. The map $X \rightarrow V(X)$ lifts to a map $\widehat{V}: \operatorname{det} \rightarrow$ $\operatorname{det}^{*}$ which is an isomorphism on the fibers. One may understand this lift in the following way. The fiber in the det* $^{*}$ bundle over a subspace $V$ can be characterized using maps which invert the projection of $V$ on $W$ up to a trace class perturbation. Such maps are called admissible frames for $V$. The natural lift $\widehat{V}$ for the map $V$ can be understood by seeing that it is possible to use an admissible frame for $V(X)$ to construct an admissible parametrix for $X$ (see [8] for more details). In any case one can use the map $\widehat{V}$ to show that a trivialization of the bundle $\operatorname{det}^{*} \rightarrow V(\mathscr{F})$ leads to a trivialization of the bundle det $\rightarrow \mathscr{F}$ (or corresponding sub-bundles if the bundle 
over $V(\mathscr{F})$ is not trivial). Furthermore $\operatorname{det}^{*}$ has a natural canonical section which pulls back to $\sigma$ under $\widehat{V}$. A trivialization in det* pulls back to a trivialization in det and since the canonical sections are also so related one can work exclusively in $\operatorname{det}^{*}$ if this proves desirable. Unfortunately, localizing the operator $D_{a, \lambda}(m)$ in the complement of $S$ produces subspaces $W_{\text {int }}(a)$ on the boundary which are too far from $W$ to be in the Grassmannian $\operatorname{Gr}(W)$. However, it turns out that for $a_{1}, \ldots, a_{n}$ in $S$ the subspaces $W(a)$ are in a Grassmannian $\mathrm{Gr}_{0}$ with reference subspace $W_{0}:=W\left(a_{1}^{0}, \ldots, a_{n}^{0}\right)$ that we will presently describe. It is still reasonable to define a determinant for $D_{a, \lambda}(m)$ by first obtaining a trivialization of the $\operatorname{det}^{*}$ bundle over the family of subspaces $W_{\text {int }}(a)$ in $\mathrm{Gr}_{0}$ and then dividing the trivializing section by the canonical section. However, because both the domain and the range of the operator $D_{a, \lambda}(m)$ depend on the parameters $a_{1}, \ldots, a_{n}$ it is difficult to define a suitable determinant bundle over the family of operators $D_{a, \lambda}(m)$. We believe that this could be done; one could, for example, define the determinant bundle as the det $^{*}$ bundle of a localization-the appropriate transfer formalism could then be used to show that the result was independent of the choice of localization. For the present we will not attempt this and we simply regard the det* formalism as an alternative which captures the essential information in our application to the Dirac operators $D_{a, \lambda}(m)$.

We will now describe the Grassmannian $\mathrm{Gr}_{0}$. Recall that $W_{\text {ext }}$ denotes the subspace of $H$ which consists of boundary values of $H^{1}\left(\mathbf{R}^{2} \backslash S\right)$ solutions, $f$, to the Dirac equation $D(m) f(z)=0$ for $z$ in the exterior of the region $S$. Theorem 3.1 tells us that $W_{0}$ and $W_{\text {ext }}$ are transverse subspaces in $H$ and we let $P_{0}$ denote the projection on $W_{0}$ relative to the splitting:

$$
H=W_{0}+W_{\text {ext }} \text {. }
$$

We are interested in the Grassmannian, $\mathrm{Gr}_{0}$, of closed subspaces of $H$ which are close to $W_{0}$ in the following sense. A closed subspace $W$ is in $\mathrm{Gr}_{0}$ provided that the map

$$
P_{0}: W \rightarrow W_{0}
$$

is Fredholm with index 0 , and the map

$$
\left(I-P_{0}\right): W \rightarrow W_{\text {ext }}
$$

is compact. Without the specification of the index for the map in (4.5) this is essentially the definition of the Grassmannian that Segal and Wilson consider in [15] and the reader can find a detailed theory of 
such Grassmannians in the book by Segal and Pressley. The restriction to index 0 in (4.5) just means that we confine our attention to the connected component of the Grassmannian containing $W_{0}$. It is simpler to discuss the det $^{*}$ bundle over the connected component of the Grassmannian and it will suffice for our purposes. The first result we require is that:

THEOREM 4.1. If $a_{\nu} \in B_{\varepsilon}\left(a_{\nu}^{0}\right)$ for $\nu=1, \ldots, n$ then $W_{\text {int }}(a) \in$ $\mathrm{Gr}_{0}$.

Proof. In what follows we suppose that $a_{\nu} \in B_{\varepsilon}\left(a_{\nu}^{0}\right)$ for $\nu=$ $1, \ldots, n$. Let $P(a)$ denote the projection of $H$ on $W_{\text {int }}(a)$ relative to the splitting $H=W_{\mathrm{int}}(a) \oplus W_{\mathrm{ext}}$ (which is a continuous direct sum as a consequence of Theorem 3.1). What we require for the proof is the analogue of the formula (3.25) in Theorem 3.3. Let $G_{l k}^{a, \lambda}\left(z, z^{\prime}\right)$ denote the $i k$ th matrix element of the Green function for $D_{a, \lambda}(m)$ introduced in $\S 3$. For $f \in H$ we claim that:

$$
(P(a) f)_{j}(z)=\int_{\partial S} G_{j 1}^{a, \lambda}\left(z, z^{\prime}\right) f_{1}\left(z^{\prime}\right) d z^{\prime}+G_{j 2}^{a, \lambda}\left(z, z^{\prime}\right) f_{2}\left(z^{\prime}\right) d \bar{z}^{\prime}
$$

for $z \in S$. To avoid introducing extra notation we will temporarily use $P(a)$ to denote the operator defined by (4.7). The result we wish to prove is simpler than Theorem 3.3 since we can use the transversality of $W_{\text {int }}(a)$ and $W_{\text {ext }}$ in $H$. We will first evaluate (4.7) on $f \in W_{\text {int }}(a)$ and then on $f \in W_{\mathrm{ext}}$. To begin suppose $f$ is in the subspace $W_{\mathrm{int}}(a)$. Then it is easy to see that the one form in (4.7) which is integrated to give $P(a) f(z)$ is exact in $S$ except for the places where $z^{\prime}=z$ or $z^{\prime}=a_{\nu}$ for some $\nu=1, \ldots, n$. Using the local asymptotics for $G^{a, \lambda}\left(z, z^{\prime}\right)$ one sees that Stokes' theorem generates a contribution only from the singularity at $z^{\prime}=z$ in the calculation of $P(a) f(z)$ and one finds:

$$
P(a) f(z)=f(z) \quad \text { for } z \in S \text { and } f \in W_{\text {int }}(a) .
$$

Next I claim that:

$$
P(a) W_{\mathrm{ext}}=0 .
$$

To see this suppose that $f \in W_{\text {ext }}$. Then the one form which one integrates to get $P(a) f$ is easily seen to be exact in the exterior of the set $S$. Thus the contour integral in the definition of $P(a) f$ on the portion of the boundary $\partial S$ which lies between two adjacent branch points $a_{\nu}$ and $a_{\nu+1}$ is seen to be zero by Stokes' theorem. The integral over the portion of the contour $\partial S$ which lies above the highest branch 
cut can be closed at $\infty$ to give zero as can the integral over the portion of the contour $\partial S$ which lies below the lowest branch cut. Thus $P(a) f=0$ for $f \in W_{\text {ext }}$. Since $H=W_{\text {int }}(a)+W_{\text {ext }}$ is a direct sum decomposition we have shown that $P(a)$ given by $(4.7)$ is the projection of $H$ on $W_{\text {int }}(a)$ along the subspace $W_{\text {ext }}$. To see that $W_{\text {int }}(a) \in \mathrm{Gr}_{0}$ we differentiate (4.7) in the variable $a$ using formulas (3.62) and (3.63) for the derivative of the Green function. One finds that $d_{a} P(a)$ is a finite rank operator. Integrating along a path which joins $a^{0}$ to $a$ while staying inside $\cup B_{\varepsilon}\left(a_{\nu}^{0}\right)$ one sees immediately that:

$$
P(a)-P_{0}=P(a)-P\left(a^{0}\right)
$$

is compact. From this fact, and the fact that $W_{\text {int }}(a)$ is joined to $W_{\text {int }}\left(a^{0}\right)$ by a continuous path it is easy to see that $W_{\text {int }}(a)$ is in the Grassmannian $\mathrm{Gr}_{0}$.

Recall that an invertible linear map $F: W_{0} \rightarrow W$ is an admissible frame for the subspace $W \in \mathrm{Gr}_{0}$ provided that $P_{0} F: W_{0} \rightarrow W_{0}$ is a trace class perturbation of the identity. We will now introduce two different admissible frames for the subspace $W_{\text {int }}(a)$. The first such frame will define the canonical section of the det $^{*}$ bundle over $\mathrm{Gr}_{0}$ and the second will be used to provide the trivialization of det $^{*}$ over the family of subspaces $a \rightarrow W_{\text {int }}(a)$ in $\mathrm{Gr}_{0}$.

First we show that the restriction $P(a): W_{0} \rightarrow W_{\text {int }}(a)$ inverts the projection $P_{0}: W_{\text {int }}(a) \rightarrow W_{0}$. From this it follows that the restriction of $P(a)$ to $W_{0}$ is an admissible frame for $W_{\text {int }}(a)$ which defines the canonical section of the $\operatorname{det}^{*}$ bundle. The argument is a simple one. Suppose that $w \in W_{0}$ and $w=w_{a}+w_{\text {ext }}$ with $w_{a} \in W_{\text {int }}(a)$ and $w_{\text {ext }} \in W_{\text {ext }}$. Then $P(a) w=w_{a}$ and writing $w_{a}=w-w_{\text {ext }}$ we see that $P_{0} w_{a}=w$.

The second frame for $W_{\text {int }}(a)$ arises from considering the direct sum of "one point" projections in the following manner. Suppose for the moment that $a_{k}$ is the only branch point and write $W_{\text {int }}\left(a_{k}\right)$ for the corresponding subspace of $H_{k}:=H^{1 / 2}\left(\partial S_{k}\right)$ and $W_{\text {ext }, k}$ for the boundary values on $\partial S_{k}$ of $H^{1}$ solutions to $D(m) f(z)=0$ for $z$ in the exterior of $S_{k}$. Now let $P\left(a_{k}\right)$ denote the projection of $H^{1 / 2}\left(\partial S_{k}\right)$ onto $W_{\text {int }\left(a_{k}\right)}$ relative to the splitting $H_{k}=W_{\text {int }}\left(a_{k}\right) \oplus W_{\text {ext }, k}$. Write $F(a)=P\left(a_{1}\right) \oplus \cdots \oplus P\left(a_{n}\right)$ for the direct sum of these projections acting on $H=H_{1} \oplus \cdots \oplus H_{n}$.

Proposition 4.2. The restriction of $F(a)$ to the subspace $W_{0}$ is an admissible frame for $W_{\mathrm{int}}(a)$. 
Proof. The first thing to check is that the restriction of $F(a)$ to the subspace $W_{0}$ is an isomorphism onto $W_{\text {int }}(a)$. When there is just one branch point (say $a_{\nu}$ ) then, as has already been observed, the restriction of $P\left(a_{\nu}\right)$ to the subspace $W_{\text {int }}\left(a_{\nu}\right)$ inverts the projection

$$
P\left(a_{\nu}^{0}\right): W_{\text {int }}\left(a_{\nu}\right) \rightarrow W_{\text {int }}\left(a_{\nu}^{0}\right)
$$

which is an isomorphism since the complementary subspace $W_{\text {ext }, k}$ is transverse to both subspaces $W_{\text {int }}\left(a_{\nu}\right)$ and $W_{\text {int }}\left(a_{\nu}^{0}\right)$. Since $W_{0}=$ $\sum_{\nu} W_{\text {int }}\left(a_{\nu}^{0}\right)$ and $W_{\text {int }}(a)=\sum_{\nu} W_{\text {int }}\left(a_{\nu}\right)$ the map $F(a)$ (which acts diagonally with respect to these direct sum decompositions) is clearly an isomorphism in the $n$-point case as well. Thus to prove that $F(a)$ is an admissible frame it will suffice to show that the restriction of $P_{0} F(a)$ to the subspace $W_{0}$ is a trace class perturbation of the identity on $W_{0}$. Since the restriction of $P_{0} F\left(a^{0}\right)$ to $W_{0}$ is the identity it is enough to show that the difference $F(a)-F\left(a^{0}\right)$ is trace class. Using the formula for the derivative of the one-point Green function found in $\S 2$ one sees that $d_{a} F(a)$ is finite rank. The operator $F(a)-F\left(a^{0}\right)$ is recovered from this derivative by integrating along a path, $\gamma$, from $a^{0}$ to $a$ which stays inside the union of the balls $B_{\varepsilon}\left(a_{\nu}^{0}\right)$. The resulting integral clearly converges in the Schmidt norm since the kernel of the finite rank operator $d_{a} F(a)$ is square integrable on $\partial S$ with a uniform bound for the integral of the square when $a_{\nu} \in B_{\varepsilon^{\prime}}\left(a_{\nu}^{0}\right)$ for any $\varepsilon^{\prime}<\varepsilon$. Thus the integral along $\gamma$ of the squares of the eigenvalues of $d_{a} F(a)$ are finite. Since $L^{1}(\gamma) \subset L^{2}(\gamma)$ and $d_{a} F(a)$ has rank $2 n$ it follows that the $L^{1}(\gamma)$ norms of the eigenvalues for $d_{a} F(a)$ are summable. This shows that integral of $d_{a} F(a)$ along $\gamma$ converges in trace norm and finishes the proof of the proposition.

We now recall that the fiber in the $\operatorname{det}^{*}$ bundle over a subspace $V \in \mathrm{Gr}_{0}$ can be identified with equivalence classes of pairs $(v, \alpha)$ where $v: W_{0} \rightarrow V$ is an admissible frame and $\alpha$ is a complex number. The equivalence relation which defines the fiber is $\left(v_{1}, \alpha_{1}\right)=\left(v_{2}, \alpha_{2}\right)$ if and only if:

$$
\alpha_{1}=\alpha_{2} \operatorname{det}\left(v_{2}^{-1} v_{1}\right)
$$

In this representation the canonical section of the $\operatorname{det}^{*}$ bundle is given by $V \rightarrow\left(v, \operatorname{det}\left(P_{0} v\right)\right)$ where the determinant is understood to be the determinant of $P_{0} v$ regarded as a map from $W_{0}$ to $W_{0}$. Since $P_{0} P(a)$ is the identity on $W_{0}$ it follows that we may regard $W_{\text {int }}(a) \rightarrow$ $(P(a), 1)$ as a representation of the canonical section $\sigma\left(W_{\text {int }}(a)\right)$. We 
now use $F(a)$ to define a trivialization of the $\operatorname{det}^{*}$ bundle over the family of subspaces $W_{\text {int }}(a)$. Define:

$$
\delta\left(W_{\mathrm{int}}(a)\right)=\left(\left.F(a)\right|_{W_{0}}, 1\right) .
$$

We may then define a determinant $\tau\left(a, a_{0}\right)$ for the Dirac operator $D_{a, \lambda}(m)$ as follows:

$$
\begin{aligned}
\tau\left(a, a_{0}\right) & =\frac{\sigma\left(W_{\text {int }}(a)\right)}{\delta\left(W_{\text {int }}(a)\right)}=\operatorname{det}_{W_{0}}\left(\left.P(a)\right|_{W_{0}} ^{-1} F(a)\right) \\
& =\operatorname{det}_{W_{0}}\left(\left.F(a)\right|_{W_{0}} ^{-1} P(a)\right)^{-1}
\end{aligned}
$$

where we have included the second version of the tau function as a reciprocal since in this form it will be slightly simpler to relate it to the formula (4.2) for the logarithmic derivative that appears above. The principal result of this paper can now be stated.

THEOREM 4.3. The $\tau$-function defined by (4.9) has the logarithmic derivative:

$$
d \log (\tau)=\frac{m}{2} \sum_{\nu=1}^{n}\left\{a_{1 / 2, \nu}^{\nu}(-\lambda) d a_{\nu}+\overline{a_{1 / 2, \nu}^{\nu}(\lambda)} d \bar{a}_{\nu}\right\} .
$$

Proof. For simplicity write $F=\left.F(a)\right|_{W_{0}}$ in the following calculation. Let $d$ denote exterior differentiation with respect to the parameters $\left\{a_{1}, \ldots, a_{n}\right\}$. To start with, the well known rule for differentiating determinants gives (for the second determinant in (4.9) above):

$$
d \log \tau\left(a, a_{0}\right)=-\operatorname{Tr}_{W_{0}}\left(d\left(F^{-1} P(a)\right) P(a)^{-1} F\right)
$$

where the trace is calculated on $W_{0}$ and it is also understood that $P(a)$ is restricted to $W_{0}$ so that it has an inverse. But now we know that $P_{0}$ inverts $P(a)$ restricted to $W_{0}$. Thus

$$
P(a)^{-1} F=P_{0} F \text {. }
$$

In a similar fashion $F_{0}:=\bigoplus_{\nu} P\left(a_{\nu}^{0}\right)$ inverts $F$ restricted to $W_{0}$. Thus we find:

$$
d \log \tau\left(a, a_{0}\right)=-\operatorname{Tr}_{W_{0}}\left(F_{0} d(P(a)) P_{0} F\right) .
$$

But $P(a)\left(I-P_{0}\right)=0$ so that $d(P(a)) P_{0}=d P(a)$ now regarded as maps on all of $H$ and the range of $F_{0}$ is $W_{0}$ so we can remove the subspace restriction on the trace to find:

$$
d \log \tau\left(a, a_{0}\right)=-\operatorname{Tr}\left(d(P(a)) F F_{0}\right) .
$$


But now for the same reason that $P(a) P_{0}=P(a)$ on $H$ we have that $F F_{0}=F$ on $H$. Finally then we see that:

$$
d \log \tau\left(a, a_{0}\right)=-\operatorname{Tr}(d(P(a)) F) .
$$

Consulting the representation for $P(a)$ in terms of the Green function we see that this is precsely the representation for $d \log \tau$ that one finds in (4.2) above. One may follow the arguments leading to (4.3) to obtain (4.10) above.

The reader might note that although $\tau\left(a, a_{0}\right)$ certainly depends on $a_{0}$ as well as $a$ the logarithmic derivative of $\tau\left(a, a_{0}\right)$ does not depend on $a_{0}$. This will be explained more fully in the next subsection where an explicit formula for $\tau\left(a, a_{0}\right)$ as a Fredholm determinant will be obtained.

A transfer matrix calculation of $\tau$. Here we will use the transfer formalism of $\S 3$ to obtain a more explicit formula for the determinant which defines the $\tau$-function. The formula we wish to make more explicit is:

$$
\tau\left(a ; a_{0}\right)=\operatorname{det}_{W_{0}}\left(\left.P(a)\right|_{W_{0}} ^{-1} F(a)\right)
$$

given in (4.9) above. To begin we recall the splitting of the Hilbert space on the boundary given by (3.32). If $g \in W(a)$ then we have (in the notation of (3.44)):

$$
\left.g\right|_{\partial S_{j}}=g_{j} \oplus N_{j}(a) g_{j}
$$

and in a similar fashion for $f \in W\left(a_{0}\right)$ we have:

$$
\left.f\right|_{\partial S_{j}}=f_{j} \oplus N_{j}\left(a_{0}\right) f_{j} \text {. }
$$

A little calculation now shows that $F(a)$ maps the element $f \in W\left(a_{0}\right)$ given by (4.12) into the element in $W(a)$ with $j$ th boundary value:

$$
f_{j} \oplus N_{j}(a) f_{j} \text {. }
$$

Thus in terms of the coordinates $\left(f_{1}, f_{2}, \ldots, f_{n}\right)$ and $\left(g_{1}, g_{2}, \ldots\right.$, $\left.g_{n}\right)$ for $W\left(a_{0}\right)$ and $W(a)$ defined by (4.12) and (4.11) above the map $F(a): W\left(a_{0}\right) \rightarrow W(a)$ is represented by the identity transformation. Next we wish to find the representation of the transformation $P(a): W\left(a_{0}\right) \rightarrow W(a)$ in these same coordinates. For this purpose suppose that $f \in W\left(a_{0}\right)$ and that one wants to obtain a splitting $f=g+h$ where $g \in W(a)$ and $h \in W_{\text {ext }}$ (so that $\left.g=P(a) f\right)$. Following the calculation from (3.41) to (3.57) but this time keeping track of what happens to $f$ on the right-hand side and making use of 
the fact that $f \in W\left(a_{0}\right)$ in the same way that the calculation uses the fact that $g \in W(a)$ we find:

$$
(I+M(a)) g=\left(I+M\left(a_{0}\right)\right) f
$$

where $g$ denotes the column vector with $j$ th entry $g_{j}, f$ denotes the column vector with $j$ th entry $f_{j}$ and:

$$
M(a)=\left[\begin{array}{ccccc}
0 & U_{1}(a) & 0 & \cdot & 0 \\
L_{2}(a) & 0 & U_{2}(a) & \cdot & 0 \\
\cdot & \cdot & \cdot & \cdot & \cdot \\
0 & \cdot & L_{n-1}(a) & 0 & U_{n-1}(a) \\
0 & \cdot & \cdot & L_{n}(a) & 0
\end{array}\right]
$$

Thus we find that:

$$
\begin{aligned}
\tau\left(a, a_{0}\right) & =\operatorname{det}\left\{\left(I+M\left(a_{0}\right)\right)^{-1}(I+M(a))\right\} \\
& =\operatorname{det}\left\{(I+M(a))\left(I+M\left(a_{0}\right)\right)^{-1}\right\} .
\end{aligned}
$$

Now in the notation of (3.58) and (3.59) let $\widetilde{M}(a)$ denote the matrix $M(a)$ with $U_{j}(a)$ replaced by $U_{j}^{0}(a)$ and $L_{j}(a)$ replaced by $L_{j}^{0}(a)$. We have seen above that the matrix elements of $\widetilde{M}\left(a_{0}\right)$ and $\widetilde{M}(a)$ differ by trace class operators. Thus the determinant

$$
\operatorname{det}\left\{\left(I+\widetilde{M}\left(a_{0}\right)\right)(I+\widetilde{M}(a))^{-1}\right\}
$$

converges in the usual sense. Indeed the upper triangular-lower triangular factorization that is described following (3.60) shows that the determinant defined in (4.15) is equal to 1 . We now multiply the formula for $\tau\left(a, a_{0}\right)$ that we found above by (4.15) and use the multiplicative property of determinants to obtain:

$$
\begin{aligned}
\tau\left(a, a_{0}\right) & =\operatorname{det}\left\{\left(I+\widetilde{M}\left(a_{0}\right)\right)(I+\widetilde{M}(a))^{-1}(I+M(a))\left(I+M\left(a_{0}\right)\right)^{-1}\right\} \\
& =\operatorname{det}\left\{(I+\widetilde{M}(a))^{-1}(I+M(a))\left(I+M\left(a_{0}\right)\right)^{-1}\left(I+\widetilde{M}\left(a_{0}\right)\right)\right\} .
\end{aligned}
$$

We would like to use the multiplicative property of determinants again to write:

$$
\tau\left(a, a_{0}\right)=\frac{\tau(a)}{\tau\left(a_{0}\right)}
$$

where:

$$
\tau(a)=\operatorname{det}\left\{(I+\widetilde{M}(a))^{-1}(I+M(a))\right\} .
$$

This is almost legitimate except that $M(a)$ and $\widetilde{M}(a)$ differ by an operator which is clearly Schmidt class but not so obviously trace class. One can get around this by defining:

$$
\tau(a)=\operatorname{det}_{2}\left\{(I+\widetilde{M}(a))^{-1}(I+M(a))\right\}
$$


where $\operatorname{det}_{2}(I+K)=\operatorname{det}\left(e^{-K}(I+K)\right)$ is the regularized determinant. The regularized determinant is not multiplicative in general so this might seem to have defeated the desired factorization in (4.16). However, using the explicit inverse of $(I+\widetilde{M}(a))$ that can be computed from the upper-lower factorzation described above we will see that:

$$
K(a):=(I+\widetilde{M}(a))^{-1}(M(a)-\widetilde{M}(a))
$$

is the Schmidt norm limit of finite rank operators, $K_{m}(a)$, which are manifestly trace 0 . Suppose that this is the case for the moment. Then since:

$$
\operatorname{det}\left(e^{F}\right)=\exp (\operatorname{Tr}(F))
$$

when $F$ is finite rank, it follows that:

$$
\operatorname{det}\left(e^{K_{m}(a)}\right)=1 .
$$

Thus

$$
\begin{aligned}
\tau\left(a, a_{0}\right) & =\operatorname{det}\left((I+K(a))\left(I+K\left(a_{0}\right)\right)^{-1}\right) \\
& =\operatorname{det}\left(e^{K_{m}\left(a_{0}\right)-K(a)}(I+K(a))\left(I+K\left(a_{0}\right)\right)^{-1}\right) .
\end{aligned}
$$

When we construct the sequence $K_{m}(a)$ we will see that it is simple to arrange that $K_{m}(a)-K_{m}\left(a_{0}\right)$ converges to $K(a)-K\left(a_{0}\right)$ in trace norm. Thus in the limit $m \rightarrow \infty$ we can replace $K_{m}\left(a_{0}\right)-K_{m}(a)$ in the preceding equation by $K\left(a_{0}\right)-K(a)$. Take this limit in the preceding equation, make the obvious similarity transformation by $e^{K\left(a_{0}\right)}$, and use the multiplicative property of determinants to obtain:

$$
\tau\left(a, a_{0}\right)=\frac{\operatorname{det}\left(e^{-K(a)}(I+K(a))\right)}{\operatorname{det}\left(e^{-K\left(a_{0}\right)}\left(I+K\left(a_{0}\right)\right)\right)}=\frac{\tau(a)}{\tau\left(a_{0}\right)}
$$

where $\tau(a)$ is defined by (4.17) above. It only remains to confirm the existence of an appropriate approximating sequence $K_{m}(a)$. First we calculate the inverse for the operator $(I+\widetilde{M}(a))$ by using the Neumann series for the upper and lower diagonal pieces of the factorization described following (3.60). One finds:

$$
(I+\widetilde{M}(a))^{-1}=\left[\begin{array}{ccccc}
I & U_{12} & U_{13} & \cdot & U_{1 n} \\
L_{21} & I & U_{23} & \cdot & U_{2 n} \\
\cdot & \cdot & \cdot & \cdot & \cdot \\
L_{n-1,1} & \cdot & L_{n-1, n-2} & I & U_{n-1, n} \\
L_{n, 1} & \cdot & L_{n, n-2} & L_{n, n-1} & I
\end{array}\right]
$$

where:

$$
L_{j k}=(-1)^{\jmath-k} L_{\jmath}^{0} \cdots L_{k-1}^{0} \quad \text { for } k<j
$$


and

$$
U_{j k}=(-1)^{k-j} U_{j}^{0} \cdots U_{k-1}^{0} \text { for } k>j .
$$

Next we calculate $M(a)-\widetilde{M}(a)$ to get:

$$
M(a)-\widetilde{M}(a)=\left[\begin{array}{ccccc}
0 & \Delta U_{1} & 0 & \cdot & 0 \\
\Delta L_{2} & 0 & \Delta U_{2} & \cdot & 0 \\
. & \cdot & \cdot & . & \cdot \\
0 & \cdot & \Delta L_{n-1} & 0 & \Delta U_{n-1} \\
0 & . & 0 & \Delta L_{n} & 0
\end{array}\right]
$$

where:

$$
\Delta L_{j}=L_{j}-L_{j}^{0}=-\left[\begin{array}{cc}
0 & 0 \\
e^{-\Delta y_{j} \omega} \alpha_{j-1} & 0
\end{array}\right]
$$

and

$$
\Delta U_{j}=U_{j}-U_{j}^{0}=-\left[\begin{array}{cc}
0 & e^{-\Delta y_{j+1} \omega} \delta_{j+1} \\
0 & 0
\end{array}\right] .
$$

Now multiplying (4.21) on the left by (4.18) one and making note of the fact that the upper triangular part of $(I+\widetilde{M}(a))^{-1}$ kills the lower triangular part of $M(a)-\widetilde{M}(a)$ and the lower triangular part of $(I+\widetilde{M}(a))^{-1}$ kills the upper triangular part of $M(a)-\widetilde{M}(a)$, one sees that the product, $K(a)$, has zeros on the diagonal. Now let $P_{m}$ denote any sequence of orthgonal projections on $H$ which tends strongly to the identity as $m \rightarrow \infty$ and write:

$$
\mathbf{P}_{m}=P_{m} \oplus P_{m} \oplus \cdots \oplus P_{m} \quad(n \text { copies }) .
$$

Then the finite rank operator $K_{m}(a):=K(a) \mathbf{P}_{m}$ also has zeros on the diagonal and so has trace 0 . The operator $K_{m}(a)$ tends to $K(a)$ in Schmidt norm since $K(a)$ is Schmidt class, and $K_{m}(a)-K_{m}\left(a_{0}\right)=$ $\left(K(a)-K\left(a_{0}\right)\right) \mathbf{P}_{m}$ tends to $K(a)-K\left(a_{0}\right)$ in trace norm since this last operator is trace class. This finishes the proof of (4.16) and (4.17). Notice that the formula for the logarithmic derivative of $\tau\left(a, a_{0}\right)$ obtained above also gives the same formula for $d_{a} \log (\tau(a))$ since:

$$
d_{a} \log (\tau(a))=d_{a} \log \left(\tau\left(a, a_{0}\right)\right)
$$

In this form the result can be used to make a conection between the lattice scaling results of [7] and the deformation theory described in the next section.

Specializing to the two-point case and using the formulas (3.34)(3.37) we can obtain an even more explicit representation. Suppose 
that $a_{1}$ and $a_{2}$ differ only in their second coordinate by $r>0$ and that $\lambda_{1}$ and $\lambda_{2}$ are the associated exponents of monodromy. Then we write $\tau(r)$ for $\tau\left(a_{1}, a_{2}\right)$ (in the last section we will see that $\tau\left(a_{1}, a_{2}\right)$ does indeed only depend on $\left.\left|a_{1}-a_{2}\right|\right)$ and one finds after a little calculation that:

$$
\tau(r)=\operatorname{det}\left(I-K_{\lambda_{1}, \lambda_{2}}^{r}\right)
$$

where $K_{\lambda_{1}, \lambda_{2}}^{r}$ is the integral operator on $L^{2}(0, \infty)$ with kernel $K_{\lambda_{1}, \lambda_{2}}^{r}(s, t)$ given by:

$$
\frac{s_{\lambda_{1}} s_{\lambda_{2}}}{\pi^{2}} \int_{0}^{\infty} d u\left\{\frac{e^{-(r / 2) \omega(s)-r \omega(u)-(r / 2) \omega(t)}}{(s+u)(t+u)}\left(\frac{u}{s}\right)^{\lambda_{2}}\left(\frac{t}{u}\right)^{\lambda_{1}}\right\} .
$$

Such an explicit formula is, finally, the payoff for the labor that went into the demonstration of Proposition 3.4 and the calculation of the one-point Green function.

Note added in proof. See [21] for an analysis of the asymptotics of this $\tau$-function for small $r$.

\section{The deformation equations.}

Existence results for $W_{j}$ and $W_{j}^{*}$. In this section we will deduce the SMJ deformation equations for the "low order expansion" coefficients of the wave functions $W_{j}$ and $W_{j}^{*}$ and the formulas which relate the tau function to these expansion coefficients. We begin by explaining some existence results derived in SMJ III and the bearing that these results have on the existence of the wave function $W_{j}(\lambda)$ and $W_{j}^{*}(\lambda)$. In SMJ III it is proved that the space of solutions to the Dirac equation branched at $\left\{a_{1}, \ldots, a_{n}\right\}$ with monodromy $e^{-2 \pi i \lambda_{j}}$ at $a_{j}$ (with $0<\left|\lambda_{j}\right|<\frac{1}{2}$ ) and globally in $L^{2}\left(\mathbf{R}^{2}\right)$ is $n$-dimensional. They construct a canonical basis $\left\{w_{j}(L)\right\}$ for this space (see Theorem 3.2.8 and equation (3.2.19)). In their work the parameter $L$ is a diagonal matrix with $j$ th entry $l_{j}$. The $l_{j}$ are related to our parameter $\lambda_{j}$ in the following manner:

$$
l_{j}= \begin{cases}\lambda_{j}+\frac{1}{2} & \text { for } \lambda_{j}<0 \\ \lambda_{j}-\frac{1}{2} & \text { for } \lambda_{j}>0\end{cases}
$$

We will write $W(A, L)$ for the $n$-dimensional space spanned by the $\left\{w_{j}(L)\right\}$. In the notation of $\S 1$ following (1.12) the basis $\left\{w_{j}(L)\right\}$ is characterized by the condition:

$$
c_{\nu}\left(w_{\mu}(L)\right)=\delta_{\mu \nu}
$$


on the local expansion coefficients. Proposition 1.3 shows that these wave functions are uniquely characterized by condition (5.2).

We will now use this existence result to deduce existence results for the wave functions $W_{j}(-\lambda)$ each of which is square integrable at $\infty$ and characterized by the local expansions:

$$
\begin{aligned}
W_{j}(-\lambda)= & \delta_{j \nu} w_{-1 / 2-\lambda_{\nu}} \\
& +\sum_{k>0}\left\{a_{k j}^{\nu}(-\lambda) w_{k-\lambda_{\nu}}+b_{k j}^{\nu}(-\lambda) w_{k+\lambda_{\nu}}^{*}\right\} .
\end{aligned}
$$

Recall that $\mathbf{N}$ is the subset of $\{1, \ldots, n\}$ characterized by the condition that $\lambda_{j}<0$ if $j \in N$ and $\mathbf{P}$ is the subset of $\{1, \ldots, n\}$ characterized by the condition that $\lambda_{j}>0$ if $j \in \mathbf{P}$. If $j \in N$ then it is easy to see that $W_{j}(-\lambda)$ must be globally in $L^{2}$ and so ought to be expressible in terms of the SMJ basis $\left\{w_{\nu}(L)\right\}$. Without difficulty one may verify:

$$
c_{\nu}\left(W_{j}(-\lambda)\right)=\delta_{\nu j} \quad \text { for } \nu, j \in \mathbf{N}
$$

and

$$
c_{\nu}^{*}\left(W_{j}(-\lambda)\right)=0 \quad \text { for } \nu \in \mathbf{P}, \text { and } j \in \mathbf{N} .
$$

These conditions characterize the expansion (5.3) for $W_{j}(-\lambda)$ when $j \in \mathbf{N}$. Thus to show that $W_{j}(-\lambda)$ exists for $j \in \mathbf{N}$ it is enough to show that there is an element of $W(A, L)$ which satisfies (5.4) and (5.5). But Proposition 1.3 shows that if $w \in W(A, L)$ and $c_{\nu}(w)=0$ for $\nu \in \mathbf{N}$ and $c_{\nu}^{*}(w)=0$ for $\nu \in \mathbf{P}$ then $w=0$. Thus the $n$ linear functionals $\left\{c_{\nu}\right\}_{\nu \in \mathbf{N}} \cup\left\{c_{\nu}^{*}\right\}_{\nu \in \mathbf{P}}$ are linearly independent on $W(A, L)$ and it follows that it is possible to satisfy (5.4) and (5.5) with an element from $W(A, L)$. Now suppose that $j \in \mathbf{P}$. Then using (1.2) and (1.3) to differentiate the local expansions for $w_{j}(L)$ one sees that $\frac{2}{m} \partial_{z} w_{j}(L)$ has a local expansion which fails to be of type (5.3) only at points $a_{\nu}$ with $\nu \in \mathbf{N}$. At these points we can subtract a suitable multiple of $w_{\nu}(L)$ to insure that the expansion has the correct form (5.3). Thus for the right choice of constants $d_{k}$ with $k \in \mathbf{N}$ (these constants are just certain expansion coefficients of $\left.w_{j}(L)\right)$ one finds that:

$$
\frac{2}{m} \partial_{z} w_{j}(L)-\sum_{k \in \mathbf{N}} d_{k} w_{k}(L)
$$

has an expansion of type (5.3) at each of the points $a_{\nu}$. This finishes the translation of the existence results for the canonical basis 
$w_{j}(L)$ into existence results for $W_{j}(-\lambda)$. The solutions $W_{j}^{*}(-\lambda)$ are obtained from $W_{j}(-\lambda)$ by the conjugation:

$$
\left[\begin{array}{l}
f \\
g
\end{array}\right] \rightarrow\left[\frac{\bar{g}}{f}\right]
$$

and this completes our discussion of existence results for $W_{j}(-\lambda)$ and $W_{j}^{*}(-\lambda)$.

Algebraic relations among the expansion coefficients. Following SMJ III we will now deduce relations among the low order expansion coefficients of $W_{j}$ and $W_{j}^{*}$ by differentiating the local expansions for $W_{j}$ and $W_{j}^{*}$ with respect to $z$ and using the uniqueness result of Proposition 1.4. The simplest of these relations is:

$$
\bar{\partial} W_{j}(-\lambda)-\frac{m}{2} \sum_{\mu} b_{1 / 2, j}^{\mu}(-\lambda) W_{\mu}^{*}(\lambda)=0
$$

This is obtained by simply noting that the local expansion of the lefthand side is of type (1.5) at each point $a_{\nu}$.

Now write $b_{0}(\lambda)$ for the $n \times n$ matrix whose $j \nu$ th entry is:

$$
b_{0}(\lambda)_{j \nu}=b_{1 / 2, j}^{\nu}(\lambda) \text {. }
$$

Then the fact that the local expansion coefficient for $w_{1 / 2-\lambda_{\nu}}$ must vanish at $a_{\nu}$ in (5.7) implies:

$$
b_{0}(-\lambda) \bar{b}_{0}(\lambda)=I
$$

where $I$ is the $n \times n$ identity matrix. Setting the local expansion coefficient of $w_{1 / 2-\lambda_{\nu}}^{*}$ at $a_{\nu}$ to 0 in (5.7) one finds:

$$
b_{1}(-\lambda)=b_{0}(-\lambda) \bar{a}_{0}(\lambda)
$$

where $b_{1}(\lambda)$ is the $n \times n$ matrix whose $j \nu$ entry is $b_{3 / 2, j}^{\nu}(\lambda)$ and $a_{0}(\lambda)$ is the $n \times n$ matrix whose $j \nu$ entry is $a_{1 / 2, j}^{\nu}(\lambda)$ (we have shifted the indices down $1 / 2$ to avoid half integer indices).

A slightly more complicated relation is obtained by noting the action of the infinitesimal rotation $R=R_{\nu}+a_{\nu} \partial-\bar{a}_{\nu} \bar{\partial}$ on the local expansions of $W_{j}(\lambda)$. One finds that subtracting $a_{j} \partial W_{j}(-\lambda)$ from $R W_{j}(-\lambda)$ kills the lowest local expansion coefficient at level $k=$ $-3 / 2$. The remaining local expansion coefficients at level $k=-1 / 2$ can all be canceled by the suitable subtraction of a linear combination of the wave functions $W_{\mu}(-\lambda)$ and $W_{\mu}^{*}(\lambda)$. One finds:

$$
\begin{aligned}
\left(R-a_{j} \partial\right) W_{j}(-\lambda) & +\sum_{\mu}\left(f_{j \mu}+\frac{1}{2} \delta_{j \mu}\right) W_{\mu}(-\lambda) \\
& +\sum_{\mu} \bar{A}_{\mu} b_{0}(-\lambda)_{j \mu} W_{\mu}^{*}(\lambda)=0
\end{aligned}
$$


where we have written:

$$
A_{\mu}:=\frac{m a_{\mu}}{2} .
$$

Writing $A$ for the diagonal matrix with $\mu \mu$ entry $A_{\mu}$ and $\Lambda$ for the diagonal matrix with $\mu \mu$ matrix element $\lambda_{\mu}$, the matrix $f$ which appears in (5.10) is defined by:

$$
f:=\Lambda+\left[A, a_{0}(-\lambda)\right]
$$

where $[A, B]:=A B-B A$ denotes the usual commutator.

There is interesting non-trivial information concerning the low order expansion coefficients $a_{0}(\lambda)$ and $b_{0}(\lambda)$ for $W_{j}$ and $W_{j}^{*}$ at level $k=\frac{1}{2}$ in local expansions for (5.10). Calculating the coefficient of $w_{1 / 2-\lambda_{\nu}}$ one finds:

$$
\begin{aligned}
{\left[A, a_{1}(-\lambda)\right]=} & a_{0}(-\lambda)+\left[\Lambda, a_{0}(-\lambda)\right]+\left[A, a_{0}(-\lambda)\right] a_{0}(-\lambda) \\
& +b_{0}(-\lambda) \bar{A} \bar{b}_{0}(\lambda)-\bar{A}
\end{aligned}
$$

A further relation is obtained by calculating the coefficient of $w_{1 / 2+\lambda_{\nu}}^{*}$ in the local expansions of (5.10) at $a_{\nu}$ :

$$
f b_{0}(-\lambda)=b_{0}(-\lambda)\left(\Lambda+\left[\bar{a}_{0}(\lambda), \bar{A}\right]\right)
$$

where (5.9) has been used to eliminate the appearance of $b_{1}(-\lambda)$ in this relation.

Deformation equations. We now follow SMJ and deduce deformation equations (in the variables $a_{\nu}$ ) for the matrices $f$ and (a slight modification of ) $b_{0}(-\lambda)$. The reason for choosing these matrices has to do with the observation that the wave functions $W_{j}(-\lambda)$ and $W_{j}^{*}(\lambda)$ satisfy a linear holonomic system in the $a$ variables with the entries of $f$ and $b_{0}(-\lambda)$ as coefficients. This is worked out in detail in SMJ III for the canonical case and we could follow a similar course here using the uniqueness result to express $a$ derivatives of $W_{j}(-\lambda)$ and $W_{j}^{*}(\lambda)$ in terms of $z$ derivatives and the wave functions themselves.

Instead we will deduce the deformation equations from the observation that the one form:

$$
\sum_{j} s_{j}^{-1} W_{j}(-\lambda) \otimes W_{j}(\lambda) d a_{j}+s_{j}^{-1} W_{j}^{*}(\lambda) \otimes W_{j}^{*}(-\lambda) d \bar{a}_{j}
$$

is closed, being proportional to the derivative of the Green's function $G^{a, \lambda}$ with respect to the $a$ variables. There is not any real advantage to this but in some respect it is more straightforward to arrive at the full collection of identities which SMJ employ to deduce the deformation equations. I believe it is also conceptually interesting to realize 
that the information in the deformation equations is contained in the equation $d^{2} G^{a, \lambda}=0$, where $d$ denotes the exterior derivative:

$$
d:=\sum_{j} d a_{j} \partial_{j}+d \bar{a}_{j} \bar{\partial}_{j}
$$

with respect to the $a$ parameters. Our strategy in determining the deformation equations will be to calculate the local expansion coefficients in the exterior derivative of the one form (5.14) at site $a_{\mu}$ in the first variable and $a_{\nu}$ in the second. The following formulas for the leading terms in the local expansions for $d W_{j}(\lambda)$ and $d W_{j}^{*}(\lambda)$ are useful in doing the calculations which follow:

$$
\begin{aligned}
d W_{j}(\lambda)= & -\delta_{j \nu} d A_{\nu} w_{-3 / 2+\lambda_{\nu}}-a_{1 / 2, j}^{\nu}(\lambda) d A_{\nu} w_{-1 / 2+\lambda_{\nu}} \\
& -b_{1 / 2, j}^{\nu}(\lambda) d \bar{A}_{\nu} w_{-1 / 2-\lambda_{\nu}}^{*} \\
& +\left(d a_{1 / 2, j}^{\nu}(\lambda)-a_{3 / 2, j}^{\nu}(\lambda) d A_{\nu}-\delta_{j \nu} d \bar{A}_{\nu}\right) w_{1 / 2+\lambda_{\nu}} \\
& +\left(d b_{1 / 2, j}^{\nu}(\lambda)-b_{3 / 2, j}^{\nu}(\lambda) d \bar{A}_{\nu}\right) w_{1 / 2-\lambda_{\nu}}^{*}+\cdots
\end{aligned}
$$

and

$$
\begin{aligned}
d W_{j}^{*}(\lambda)= & -\delta_{j \nu} d \bar{A}_{\nu} w_{-3 / 2+\lambda_{\nu}}^{*}-\bar{a}_{1 / 2, j}^{\nu}(\lambda) d \bar{A}_{\nu} w_{-1 / 2+\lambda_{\nu}}^{*} \\
& -\bar{b}_{1 / 2, j}^{\nu}(\lambda) d A_{\nu} w_{-1 / 2-\lambda_{\nu}} \\
& +\left(d \bar{a}_{1 / 2, j}^{\nu}(\lambda)-\bar{a}_{3 / 2, j}^{\nu}(\lambda) d \bar{A}_{\nu}-\delta_{j \nu} d A_{\nu}\right) w_{1 / 2+\lambda_{\nu}}^{*} \\
& +\left(d \bar{b}_{1 / 2, j}^{\nu}(\lambda)-\bar{b}_{3 / 2, j}^{\nu}(\lambda) d A_{\nu}\right) w_{1 / 2-\lambda_{\nu}}+\cdots
\end{aligned}
$$

We will now list the relations which one may deduce by calculating the lowest order expansion coefficients for the exterior derivative of (5.14). Computing the coefficient of $w_{-1 / 2-\lambda_{\mu}} \otimes w_{-1 / 2+\lambda_{\nu}}$ one finds that:

$$
s^{-1} a_{0}(-\lambda)-a_{0}(\lambda)^{\tau} s^{-1}=\text { diagonal }
$$

where $s$ is the diagonal matrix whose $\mu \mu$ entry is $s_{\mu}$ and $a^{\tau}$ denotes the transpose of $a$. We write $c=$ diagonal to express the fact that the off diagonal elements of the matrix $c$ vanish. Computing the coefficient of $w_{-1 / 2+\lambda_{\mu}}^{*} \otimes w_{-1 / 2+\lambda_{\nu}}$ one finds that:

$$
s^{-1} b_{0}(-\lambda)-\bar{b}_{0}(-\lambda)^{\tau} s^{-1}=0 \text {. }
$$

Computing the coefficient of $w_{1 / 2-\lambda_{\mu}} \otimes w_{-1 / 2+\lambda_{\nu}}$ one finds that:

$$
\begin{aligned}
d a_{0}(-\lambda)= & a_{1}(-\lambda) d A+d A X-a_{0}(-\lambda) d A a_{0}(-\lambda) \\
& -b_{0}(-\lambda) d \bar{A} \bar{b}_{0}(\lambda)+d \bar{A}
\end{aligned}
$$


where the matrix $X$ is not determined by this relation but using (5.15) and (5.17) one can say that:

$$
X-s a_{1}(\lambda)^{\tau} s^{-1}=\text { diagonal }
$$

which determines the off diagonal elements of $X$. Computing the coefficient of $w_{1 / 2+\lambda_{\mu}}^{*} \otimes w_{-1 / 2+\lambda_{\nu}}$ one finds that:

$$
\begin{aligned}
d b_{0}(-\lambda)= & d A Y+s^{-1} b_{1}(-\lambda) d \bar{A}-a_{0}(\lambda)^{\tau} s^{-1} d A b_{0}(-\lambda) \\
& -\bar{b}_{0}(-\lambda)^{\tau} s^{-1} d \bar{A} \bar{a}_{0}(\lambda)
\end{aligned}
$$

Once again the matrix $Y$ is not determined by this relation but combining (5.16) and (5.19) one finds that:

$$
Y=\bar{b}_{1}(-\lambda)^{\tau} s^{-1} \text {. }
$$

We are ready now to obtain the SMJ deformation equations. In addition to the matrix $f$ already introduced we define:

$$
g=s^{-1} \bar{b}_{0}(\lambda)
$$

and the matrix of one forms $\theta$ :

$$
\theta=\left[a_{0}(-\lambda), d A\right] .
$$

The matrices $f, g$ and $\theta$ are the analogues of the matrices $F, G$ and $\Theta$ introduced by SMJ in [14-III]. Since they will not be exactly the same except in the event that the local exponent of monodromy $\lambda_{j}$ is positive for all $j$ we have altered the notation slightly. First note that the matrix elements of $\theta$ are determined by $f$ :

$$
\theta_{\mu \nu}=-f_{\mu \nu} \frac{d A_{\mu}-d A_{\nu}}{a_{\mu}-a_{\nu}} .
$$

Using the definition of $f$ one finds immediately:

$$
d f=\left[A, d a_{0}(-\lambda)\right]=\theta .
$$

Now substitute (5.17) and (5.18) in this last expression. Use (5.12) and (5.15) to eliminate $a_{1}(-\lambda)$ and $a_{1}(\lambda)^{\tau}$ from the result. One finds after a little calculation:

$$
d f=[f, \theta]+\left[g^{-1} \bar{A} g, d A\right]+\left[g^{-1} d \bar{A} g, A\right] .
$$

To get the second deformation equation we calculate $d g$ using (5.19) and (5.20). Eliminating $b_{1}(\lambda)$ from the result using (5.9) one finds after some calculation:

$$
d g=g \theta+\theta^{*} g
$$


The condition (5.16) translates directly into Hermitian symmetry for $g$ :

$$
g^{*}=g .
$$

We can also translate (5.13) using (5.8) and (5.15) into the following algebraic relation between $f$ and $g$ :

$$
g f=f^{*} g .
$$

We now come to the only formal difference between the SMJ analysis and the analysis based on the wave functions $W_{j}(-\lambda)$ and $W_{j}^{*}(\lambda)$ above. Suppose that $j$ and $k$ are both in $\mathbf{N}$. Then both $W_{j}(-\lambda)$ and $W_{k}(-\lambda)$ are in $L^{2}$ and one finds the $L^{2}$ inner product using (5.2) is $\left(\right.$ note: $\left.s_{k}(\lambda)=\sin \left(\pi \lambda_{k}\right)=-s_{k}(-\lambda)\right)$ :

$$
\left\langle W_{j}(-\lambda), W_{k}(-\lambda)\right\rangle=-\frac{4}{m}\left|s_{k}(\lambda)\right| b_{1 / 2, k}^{j}(-\lambda)
$$

and in a similar fashion if $j, k \in \mathbf{P}$ then:

$$
\left\langle W_{j}^{*}(\lambda), W_{k}^{*}(\lambda)\right\rangle=-\frac{4}{m}\left|s_{k}(\lambda)\right| \bar{b}_{1 / 2, k}^{j}(\lambda) .
$$

It follows from these relations that the $|\mathbf{P}| \times|\mathbf{P}|$ submatrix of $g$ associated with the indices in $\mathbf{P}$ is negative definite and that the $|\mathbf{N}| \times|\mathbf{N}|$ submatrix of $g^{-1}$ associated with the indices in $\mathbf{N}$ is positive definite. In the SMJ scheme the matrix $G$ is always positive definite because of its association with the canonical basis.

The two-point case. We are now able to imitate the SMJ analysis of the deformation equations in the case of two branch points. There are essentially four different cases that we must analyse. If the local exponents of monodromy $\lambda_{1}$ and $\lambda_{2}$ have the same sign then we have seen that the matrix $g$ above is either positive or negative definite. In either of these two cases the analysis of the deformation equations follows the analysis in SMJ III from (3.3.42) to (3.3.47) without essential change. If the local exponents of monodromy have opposite signs then the ansatz (3.3.42) in SMJ III is not right for the matrix $g$ we have defined. We will illustrate the analysis in the case $\lambda_{1}>0$ and $\lambda_{2}<0$. Write $\partial_{j}$ for $\partial / \partial a_{j}$ and $\bar{\partial}_{j}$ for $\partial / \partial \bar{a}_{j}$. Then:

$$
d A\left(\partial_{1}+\partial_{2}\right)=(m / 2) I
$$

and it is easy to see from this and the deformation equations (5.21) and (5.22) that:

$$
\left(\partial_{1}+\partial_{2}\right) g=0
$$


and

$$
\left(\partial_{1}+\partial_{2}\right) f=0
$$

The same results are found for the complex conjugate vector field $\bar{\partial}_{1}+$ $\bar{\partial}_{2}$ and it follows that as a consequence of the deformation equations $f$ and $g$ are functions of the difference variable $a_{2}-a_{1}$ only. We write:

$$
a_{2}-a_{1}=r e^{i \phi}
$$

for the polar representation of this difference with $\phi$ taken between 0 and $2 \pi$. Next we observe that:

$$
\partial / \partial \phi=i\left(a_{1} \partial_{1}+a_{2} \partial_{2}-\bar{a}_{1} \bar{\partial}_{1}-\bar{a}_{2} \bar{\partial}_{2}\right)
$$

so that:

$$
d A(\partial / \partial \phi)=i A, \quad d \bar{A}(\partial / \partial \phi)=-i \bar{A}
$$

and

$$
\theta(\partial / \partial \phi)=i(\Lambda-f), \quad \theta^{*}(\partial / \partial \phi)=-i\left(\Lambda-f^{*}\right)
$$

One now calculates without difficulty that:

$$
\frac{\partial g}{\partial \phi}=-[i \Lambda, g] \text { and } \frac{\partial f}{\partial \phi}=-[i \Lambda, f] \text {. }
$$

It follows from these last two results that:

$$
g(r, \phi)=e^{-i \Lambda \phi} g(r, 0) e^{i \Lambda \phi}
$$

and

$$
f(r, \phi)=e^{-i \Lambda \phi} f(r, 0) e^{i \Lambda \phi} .
$$

Since $d \log \operatorname{det} g=\operatorname{Tr}\left(g^{-1} d g\right)$ and the trace of both $\theta$ and $\theta^{*}$ are 0 one sees that the determinant of $g$ is a constant independent of both $a_{1}$ and $a_{2}$.

The Hermitian symmetry of $g$, the positive definite character of the $\mathbf{N}$ part of $g^{-1}$, and the negative definite character of the $\mathbf{P}$ part of $g$ are easily seen to imply that $\operatorname{det}(g)<0$ and $g_{11}<0$. By comparing the $g$ matrix in our formalism with the $G$ matrix in the SMJ formalism one also finds that $g_{22}>0$ (I did not see a way to obtain this relation directly). In any case these observations and (5.25) show that we can parametrize $g$ in the following manner:

$$
g(r, \phi)=c\left(\begin{array}{cc}
-\kappa \cos (\psi) & \varepsilon \sin (\psi) e^{i \lambda \phi} \\
\bar{\varepsilon} \sin (\psi) e^{-i \lambda \phi} & \kappa^{-1} \cos (\psi)
\end{array}\right)
$$

where $-c^{2}$ is the determinant of $g, \lambda:=\lambda_{2}-\lambda_{1}$, the functions $\kappa$, $\varepsilon$, and $\psi$ are all functions of $r$ alone, and $\kappa$ and $\psi$ are real while 
$\bar{\varepsilon} \varepsilon=1$. Also $\cos (\psi)>0$. The reader might want to compare this with the ansatz for $G$ in SMJ III which is (see (3.3.42)):

$$
G(r, \phi)=c\left(\begin{array}{cc}
\kappa \operatorname{sh}(\psi) & \varepsilon \operatorname{ch}(\psi) e^{i \lambda \phi} \\
\bar{\varepsilon} \operatorname{ch}(\psi) e^{-i \lambda \phi} & \kappa^{-1} \operatorname{sh}(\psi)
\end{array}\right) .
$$

Making use of (5.26) and the relation (5.24) between $f$ and $g$ one finds:

$$
f=\left(\begin{array}{cc}
\lambda_{1} & \varepsilon f_{+} e^{i \lambda \phi} \\
\bar{\varepsilon} f_{-} e^{-i \lambda \phi} & \lambda_{2}
\end{array}\right)
$$

where $f_{ \pm}$is a function of $r$ alone and making use of (5.24) we find that there is a function $\varphi$ such that:

$$
2 \kappa f_{+}=-\varphi+\lambda \tan (\psi)
$$

and

$$
2 \kappa^{-1} f_{-}=\varphi+\lambda \tan (\psi) .
$$

We now calculate the $r$ derivatives for $f$ and $g$ making use of the deformation equations and:

$$
2 r \frac{\partial}{\partial r}=\left(a_{2}-a_{1}\right)\left(\partial_{2}-\partial_{1}\right)+\left(\bar{a}_{2}-\bar{a}_{1}\right)\left(\bar{\partial}_{2}-\bar{\partial}_{1}\right)
$$

from which it follows that

$$
\theta\left(r \frac{\partial}{\partial r}\right)=\Lambda-f
$$

Thus:

$$
r \frac{\partial g}{\partial r}=g(\Lambda-f)+\left(\Lambda-f^{*}\right) g
$$

and

$$
r \frac{\partial f}{\partial r}=[f, \Lambda]+2\left[g^{-1} \bar{A} g, A\right] .
$$

To obtain this last result we used the fact that $A$ and the matrix $A^{\prime}$ obtained from $A$ by interchanging $\lambda_{1}$ and $\lambda_{2}$ on the diagonal have the same commutator with an arbitrary $2 \times 2$ matrix except for a change in sign.

After a little calculation (5.31) simplifies to the following:

$$
\begin{gathered}
\frac{\partial \varepsilon}{\partial r}=0, \\
r \frac{\partial \psi}{\partial r}=-\varphi,
\end{gathered}
$$


and

$$
r \kappa^{-1} \frac{\partial \kappa}{\partial r}=\lambda \tan ^{2}(\psi)
$$

Making use of (5.35) in (5.32) one finds:

$$
r \frac{\partial \varphi}{\partial r}=-\lambda^{2} \sec ^{2}(\psi) \tan (\psi)-\frac{m^{2} r^{2}}{2} \sin (2 \psi)
$$

and finally combining (5.36) with (5.34) one has:

$$
r \frac{\partial}{\partial r}\left(r \frac{\partial \psi}{\partial r}\right)=\lambda^{2} \sec ^{2}(\psi) \tan (\psi)+\frac{m^{2} r^{2}}{2} \sin (2 \psi) .
$$

This equation is remarkably similar to equation (5.3.46) in SMJ III except that the hyperbolic functions have been replaced by trigonometric functions reflecting the change in the ansatz for $g$ above.

This similarity persists if we evaluate the logarithmic derivative of the $\tau$-function in terms of the deformation data. Recall that:

$$
d \log (\tau)=\sum_{\nu}\left\{a_{1 / 2, \nu}^{\nu}(-\lambda) d A_{\nu}+\bar{a}_{1 / 2, \nu}^{\nu}(\lambda) d \bar{A}_{\nu}\right\} .
$$

Next observe that the diagonal part of (5.12) gives an equation for the diagonal part of $a_{0}$ in terms of the off diagonal part of $a_{0}$ and the matrix $b_{0}$. The off diagonal part of $a_{0}$ is in turn determined by $f$ and $b_{0}$ is determined by $g$. These observations and the equations (5.27) and (5.28) for $f$ and $g$ can be used in (5.38) to give:

$$
d \log (\tau)=-\frac{1}{2 r}\left(\varphi^{2}-\lambda^{2} \tan ^{2}(\psi)-m^{2} r^{2} \sin ^{2}(\psi)\right) d r .
$$

Or making use of (5.34) one finds:

$$
\frac{\partial}{\partial r} \log (\tau)=-\frac{1}{2 r}\left(r^{2}\left(\frac{\partial \psi}{\partial r}\right)^{2}-\lambda^{2} \tan ^{2}(\psi)-m^{2} r^{2} \sin ^{2}(\psi)\right)
$$

which the reader might want to compare with 4.5.42 in SMJ IV.

\section{REFERENCES}

[1] E. Barouch, B. M. McCoy and T. T. Wu, Zero-field susceptibility of the twodimensional Ising model near $T_{c}$, Phys. Rev. Lett., 31 (1973), 1409-1411. C. A. Tracy and B. M. McCoy, Neutron scattering and the correlations of the Ising model near $T_{c}$, Phys. Rev. Lett., 31 (1973), 1500-1504.

[2] A. Carey, S. Ruijsennaars and J. Wright, The massless Thirring model: positivity of Klaiber's N-point function, Comm. Math. Phys., 99 (1985), 347.

[3] R. Davey, SMJ analysis of monodromy fields, thesis, unpublished (University of Arizona 1988). 
[4] B. Malgrange, Sur les deformations isomonodromiques, in Mathématique et Physique. Séminaire de l'Ecole Normale Supérieure 1979-1982, L. B. de Monvel, A. Douady, and J. L. Verdier, eds. (Birkhäuser, Boston, 1983), 400-426.

[5] _ Deformations Isomonodromiques et Fonctions $\tau$, extract of a letter to J. B. Bost, November 28, 1986.

[6] R. Narayanan and C. A. Tracy, Holonomic quantum field theory of Bosons in the Poincaré disk and the zero curvature limit, Nuclear Phys., B340 (1990), 568-594.

[7] J. Palmer, Monodromy fields on $\mathbf{Z}^{2}$, Comm. Math. Phys., 102 (1985), 175-206.

[8] _ Determinants of Cauchy-Riemann operators as $\tau$ functions, Acta Appl. Math., 18 No. 3 (1990), 199-223.

[9] J. Palmer and C. Tracy, Two dimensional Ising correlations: the SMJ analysis, Adv. in Appl. Math., 4 (1983), 46-102.

[10] _ Monodromy preserving deformations of the Dirac operator on the hyperbolic plane, in Mathematics of Nonlinear Science: proceedings of an AMS special session held January 11-14, 1989, M. S. Berger ed., Contemp. Math., 108, 119-131.

[11] A. Pressley and G. Segal, Loop Groups, Clarendon Press, Oxford (1986).

[12] D. Quillen, Determinants of Cauchy-Riemann operators on a Riemann surface, Functional Anal. Appl., 19 (1985), 37-41.

[13] S. N. M. Ruijsenaars, The Wightman axioms for the fermionic Federbush model, Comm. Math. Phys., 87 (1982), 181-228.

[14] M. Sato, T. Miwa and M. Jimbo, Holonomic quantum fields I-V Publ. RIMS, Kyoto Univ., 14 (1978), 223-267, 15 (1979), 201-278, 15 (1979), 577-629, 15 (1979), 871-972, 16 (1980), 531-584.

[15] G. Segal and G. Wilson, Loop groups and equations of KdV type, Publ. Math. I.H.E.S., 61 (1985), 5-65.

[16] C. A. Tracy, Monodromy preserving deformation theory for the Klein-Gordon equation in the hyperbolic plane, Phys., 34D (1989), 347-365.

[17] __ Monodromy preserving deformations of linear ordinary and partial differential equations, in Solitons in Physics, Mathematics, and Nonlinear Optics, P. J. Olver, and D. H. Sattinger (eds.), Springer-Verlag, New York, (1990), 165-174.

[18] E. Witten, Quantum field theory, Grassmannians and algebraic curves, Comm. Math. Phys., 113 (1988), 529-600.

[19] T. T. Wu, B. M. McCoy, C. A. Tracy, and E. Barouch, Spin-spin correlation functions for the two dimensional Ising model: Exact theory in the scaling region, Phys. Rev. B, 13 (1976), 316-374.

[20] B. M. McCoy, C. A. Tracy, and T. T. Wu, Painleve functions of the third kind, J. Math. Phys., 18 (1977), 1058-1092

[21] C. A. Tracy, Asymptotics of a $\tau$-function arising in the two-dimensional Ising model, Comm. Math. Phys., 142 (1991), 297-311.

E. L. Basor and C. A. Tracy, Asymptotics of a tau-function and Toeplitz determinants with singular generating functions, Internat. J. Modern Physics A ?, Suppl. 1A (1992), 83-107.

Received April 16, 1991.

UNIVERSITY OF ARIZONA

TUCSON, AZ 85721 